\title{
Microcontroller-based power regulation for induction motors with power quality conditioning
}

\author{
Joshua Scott Robinson \\ West Virginia University
}

Follow this and additional works at: https://researchrepository.wvu.edu/etd

\section{Recommended Citation}

Robinson, Joshua Scott, "Microcontroller-based power regulation for induction motors with power quality conditioning" (2005). Graduate Theses, Dissertations, and Problem Reports. 1641.

https://researchrepository.wvu.edu/etd/1641

This Thesis is protected by copyright and/or related rights. It has been brought to you by the The Research Repository @ WVU with permission from the rights-holder(s). You are free to use this Thesis in any way that is permitted by the copyright and related rights legislation that applies to your use. For other uses you must obtain permission from the rights-holder(s) directly, unless additional rights are indicated by a Creative Commons license in the record and/ or on the work itself. This Thesis has been accepted for inclusion in WVU Graduate Theses, Dissertations, and Problem Reports collection by an authorized administrator of The Research Repository @ WVU. For more information, please contact researchrepository@mail.wvu.edu. 


\title{
Microcontroller-Based Power Regulation For Induction Motors With Power Quality Conditioning
}

\author{
by \\ Joshua Scott Robinson \\ Thesis submitted to the \\ College of Engineering and Mineral Resources \\ at West Virginia University \\ in partial fulfillment of the requirements \\ for the degree of \\ Master of Science \\ in \\ Electrical Engineering \\ Professor Ali Feliachi, Ph.D. \\ Professor Roy Nutter, Jr., Ph.D. \\ Professor Powsiri Klinkhachorn, Ph.D., Chair
}

Lane Department of Computer Science and Electrical Engineering

Morgantown, West Virginia

2005

Keywords: induction motor, power electronics, power quality, microcontroller, line conditioner, control systems

Copyright 2005 Joshua Scott Robinson 


\author{
Abstract \\ Microcontroller-Based Power Regulation For Induction Motors With Power Quality \\ Conditioning \\ by \\ Joshua Scott Robinson \\ Master of Science in Electrical Engineering \\ West Virginia University \\ Professor Powsiri Klinkhachorn, Ph.D., Chair
}

The focus of this thesis is to verify the power-saving characteristics of a triac-based motor controller and reduce the harmonics associated with the device by integrating a power quality conditioner. A hardware prototype of a motor controller was constructed using a microcontroller to detect the load magnitude and fire a triac accordingly. Test results showed an average decrease in active power consumption of $23.2 \%$ with a $426.6 \%$ average increase in harmonic distortion; the largest power savings occurred with light loads. A Simulink model of a line conditioner was created, verified, and adapted to utilize a digital controller. This conditioner uses a DC capacitor and inverter to produce harmonic-compensating signals, and connects to the AC source via a link reactor. The model was combined with a model of the motor controller and the complete system was simulated. Results show a successful reduction in real power consumption with harmonic distortion below $6 \%$. 


\section{Acknowledgments}

My deepest thanks to my wife, Anna, for her love and support each and every day. I could not have finished this work without her encouragement, reassurance, and confidence that I would succeed.

A very sincere thanks goes to Dr. Klinkhachorn for serving as my advisor, for his guidance, and for his advice both in and out of the classroom. His knowledge and technical abilities were a tremendous help to me throughout the course of this project.

I would like to thank my mother for raising me and for showing me the value of hard work and perseverence. She has given me unwavering support in every challenge I have undertaken, and taught me to believe in myself and follow my dreams.

Thanks to Dr. Feliachi for giving me the opportunity to work with the APERC group and for serving on my committee. I would also like to thank Dr. Nutter both for being a part of my committee and for encouraging me to pursue graduate studies.

I am grateful to have so many family members and friends who have supported me. There are too many of them to name individually, but I am thankful to each one of them for being a part of my life. 


\section{Contents}

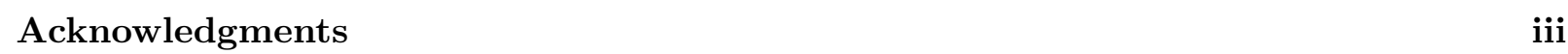

List of Figures $\quad$ vi

List of Tables $\quad$ viii

\begin{tabular}{lll}
\hline & Introduction & 1
\end{tabular}

1.1 Induction Motor Energy Consumption . . . . . . . . . . . . . . . . 1

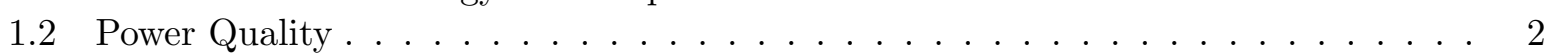

1.3 Research Goals . . . . . . . . . . . . . . . . . . . . . . . . 3

1.4 Thesis Organization $\ldots \ldots \ldots \ldots \ldots \ldots \ldots$

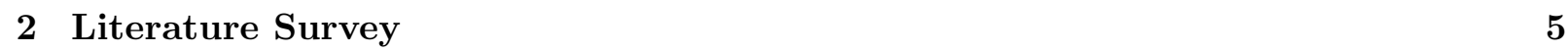

2.1 Motor Control . . . . . . . . . . . . . . . . . . . . . . . . . 5

2.2 Harmonic Filters $\ldots \ldots \ldots \ldots \ldots \ldots \ldots$

2.3 Thesis Objective $\ldots \ldots \ldots \ldots \ldots \ldots \ldots \ldots \ldots$

\begin{tabular}{llr}
\hline 3 & Motor Controller & 12 \\
\hline
\end{tabular}

3.1 Power Planner Patent . . . . . . . . . . . . . . . . . . . . . . . . . . . . . . . . . . . . . . . . .

3.2 Hardware Implementation $\ldots \ldots \ldots \ldots \ldots \ldots \ldots$

3.3 Software Design $\ldots \ldots \ldots \ldots \ldots \ldots \ldots \ldots \ldots \ldots \ldots$

3.4 Test Results . . . . . . . . . . . . . . . . . . . . . . . . . . . . . 25

3.5 Simulink Model . . . . . . . . . . . . . . . . . . . . . . . . . . . . . 31

\begin{tabular}{|lll}
\hline 4 Line Conditioner & 43
\end{tabular}

4.1 Active Power Quality Conditioner . . . . . . . . . . . . . . . . . . . . . . . . . . . .

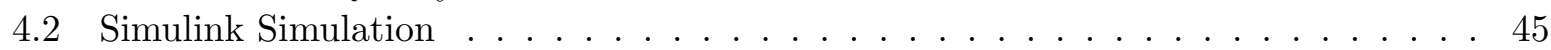

4.3 Digital Control . . . . . . . . . . . . . . . . . . . . . . . 51

4.4 Complete System Simulation $\ldots \ldots \ldots \ldots$. . . . . . . . . . . . . 59

$\begin{array}{lll}5 \text { Conclusion } & 66\end{array}$

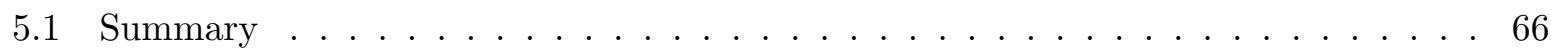

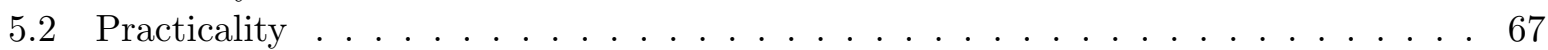

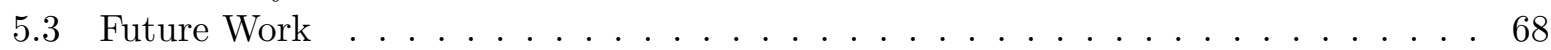

5.4 Lessons Learned $\ldots \ldots \ldots$. . . . . . . . . . . . . . . . . . . . . 69 
\begin{tabular}{ll}
\hline References & 70
\end{tabular}

\begin{tabular}{|ll}
\hline A Source Code & $\mathbf{7 2}$
\end{tabular}

A.1 PIC C Code . . . . . . . . . . . . . . . . . . . . . . . . . . 72

A.2 MATLAB Code . . . . . . . . . . . . . . . . . . . . . . 81 


\section{List of Figures}

2.1 EnergySmart Power Planner I . . . . . . . . . . . . . . . . . . . . . . . . 6

2.2 Powergy Clean Power Systems Unit _ . . . . . . . . . . . . . . . . . . . . . . 7

2.3 Low-Pass Filter Circuit Diagrams $\ldots \ldots \ldots \ldots \ldots$

3.1 Bach's Circuit Diagram $\ldots \ldots \ldots \ldots \ldots \ldots \ldots \ldots$

3.2 Inverting Amplifier Waveform . . . . . . . . . . . . . . . . . . . . . . 14

3.3 Motor Controller Circuit Diagram . . . . . . . . . . . . . . . . . . . . . . . 16

3.4 115V Load Outlet Schematic . . . . . . . . . . . . . . . . . . . . . . . . 17

3.5 Photograph of Test Load Setup ． . . . . . . . . . . . . . . . . . . . . 18

3.6 Motor Control Software Block Diagram ～. . . . . . . . . . . . . . . . . . . . 19

3.7 Motor Controller PIC Software Main Loop . . . . . . . . . . . . . . . . . . . . . . 21

3.8 PIC Software Timer0 ISR . . . . . . . . . . . . . . . . . . . . . . . . . . 23

3.9 PIC Software Analog-to-Digital ISR . . . . . . . . . . . . . . . . . . 24

3.10 Experimental Results, No Load . . . . . . . . . . . . . . . . . . . . . . . . . 27

3.11 Experimental Results, 7 in.-oz. Load . . . . . . . . . . . . . . . . . . . . 28

3.12 Experimental Results, 10 in.-oz. Load . . . . . . . . . . . . . . . . . . . . . . 29

3.13 Experimental Results, 25W Light Bulb. . . . . . . . . . . . . . . . . . . 30

3.14 Motor Controller Simulink Model . . . . . . . . . . . . . . . . . . . . . . . . . . . . 32

3.15 Triac Modeled by Thyristors . . . . . . . . . . . . . . . . . . . 33

3.16 Inverting Amplifier Block . . . . . . . . . . . . . . . . . . . . . . . . . . . . . . . . . . . .

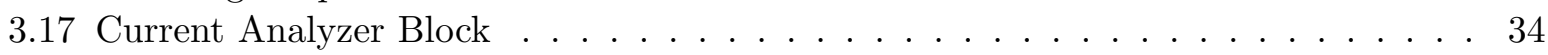

3.18 Motor Controller Block $\ldots \ldots \ldots$. . . . . . . . . . . . . . . . 35

3.19 Calculation of Firing Delay $\ldots \ldots \ldots$. . . . . . . . . . . . . 36

3.20 Gate Pulse Control Block $\ldots \ldots \ldots$. . . . . . . . . . . . . . 37

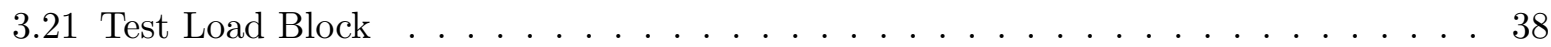

3.22 Simulated Results, No Load $\ldots . . \ldots \ldots$. . . . . . . . . . . . . 41

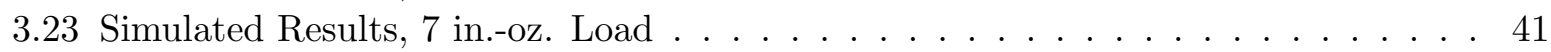

3.24 Simulated Results, 10 in.-Oz. Load . . . . . . . . . . . . . . . . . . . . . . . . . . . . . . . . . . .

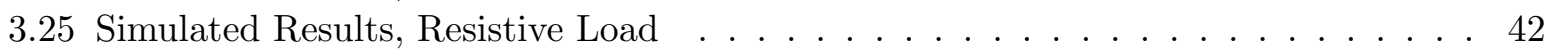

4.1 Active Power Quality Conditioner Circuit . . . . . . . . . . . . . . . . . . . . . 44

4.2 Active Power Quality Conditioner Load Detail . . . . . . . . . . . . . . . . . . . . 44

4.3 Synchronous Link Circuit . . . . . . . . . . . . . . . . . . . . . . . . 45 
4.4 Line Conditioner Simulink Model . . . . . . . . . . . . . . . . . . . . . . . . . 47

4.5 Line Conditioner Controller Block . . . . . . . . . . . . . . . . . . . . . . . . . 48

4.6 Line Conditioner Inverter Block . . . . . . . . . . . . . . . . . . . . . . . . . 49

4.7 Analog Line Conditioner Scope Traces . . . . . . . . . . . . . . . . . . . . . . 50

4.8 Load Without Line Conditioner . . . . . . . . . . . . . . . . . . . . . 51

4.9 Voltage Divider for Digital Controller . . . . . . . . . . . . . . . . . . . . . . 52

4.10 Digital Control Block . . . . . . . . . . . . . . . . . . . . . . . . 53

4.11 Lookup-Based Sine Wave Template . . . . . . . . . . . . . . . . . . . . . 54

4.12 Discrete Derivative Subsystem . . . . . . . . . . . . . . . . . . 55

4.13 Discrete PWM Block . . . . . . . . . . . . . . . . . . . . . . 56

4.14 Discrete PWM Pulse Generator . . . . . . . . . . . . . . . . . . . . 57

4.15 Digital Control Simulation Results . . . . . . . . . . . . . . . . . . . . . 58

4.16 Complete System Block Diagram . . . . . . . . . . . . . . . . . . . . . . 60

4.17 Complete System Results, No Load . . . . . . . . . . . . . . . . . . . . . . . . . 62

4.18 Complete System Results, 7 in.-oz. Load. . . . . . . . . . . . . . . . . . . . 62

4.19 Complete System Results, 10 in.-Oz. Load . . . . . . . . . . . . . . . . . . . . 63

4.20 Total Harmonic Distortion Versus Load . . . . . . . . . . . . . . . . . . . . . . 63

4.21 Real and Reactive Power Versus Load . . . . . . . . . . . . . . . . . . . . . 64 


\section{List of Tables}

3.1 Test Motor Specifications $\ldots \ldots \ldots \ldots \ldots \ldots \ldots$

3.2 Motor Controller Test Results . . . . . . . . . . . . . . . . . . . . . . . . 26

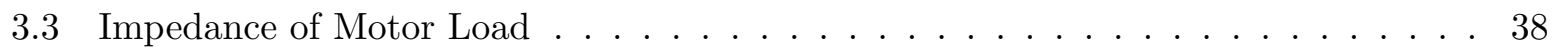

3.4 Motor Controller Simulation Results . . . . . . . . . . . . . . . . . . . . . . 40

4.1 PWM Gate Logic Truth Table . . . . . . . . . . . . . . . . . . . . . . . . . 57

4.2 Complete System Simulation Results . . . . . . . . . . . . . . . . . . . . . . . . 61

4.3 Total Power Consumption $\ldots \ldots \ldots \ldots \ldots \ldots \ldots$ 


\section{Chapter 1}

\section{Introduction}

This research focused on controlling the power consumed by an induction motor using power electronics. Additionally, regulating the quality of the power drawn by the system was investigated. Efficient use of electrical energy has always been an important issue ecologically and economically. Modern electronic devices such as personal computers are nonlinear loads that cause harmonics in the current waveform. Because of the relationship between current and voltage, current harmonics also distort the voltage waveform, leading to problems that may result in distribution system overload [1].

\subsection{Induction Motor Energy Consumption}

Induction motors account for about $45 \%$ of all energy consumed in the United States. It is also estimated that in one year of operation, a motor will consume ten times its initial cost in energy. In addition, about one third of motors currently in use operate below $50 \%$ of their rated load, consuming more energy than necessary and reducing the power factor of the system [2]. The power factor is the cosine of the phase angle difference between the voltage and current waveforms [3].

The current drawn by an induction motor has both resistive and inductive components. The magnetizing current is inductive, and is independent of the motor load; however, the resistive current is directly proportional to the load. Thus, if a motor is operating with no load, most of the current drawn is inductive and the power factor is low. As load increases, the magnetizing current remains constant but the resistive current increases, moving the power factor closer to the ideal value of 1.0 [4]. 


\subsection{Power Quality}

\subsubsection{Components of Power Quality}

When considering the power quality of a system, several factors are taken into account. Conditions that reduce the quality of power are listed and explained below [5].

- Electromagnetic interference (EMI) is any unwanted signal that affects the operation of the system. EMI may be radiated through the air or conducted through the line.

- A voltage sag is a temporary decrease in the RMS value of the AC voltage; a swell is a temporary increase. Sags and swells may last from milliseconds to several seconds.

- Harmonics are current and voltage signals with frequencies that are multiples of the fundamental frequency. When added to the fundamental signal they cause distortion of the waveform.

- The harmonic distortion factor is the magnitude of a single harmonic signal. This is equal to the RMS value of the harmonic divided by the RMS value of the fundamental, usually expressed as a percentage.

- Total harmonic distortion (THD) is the RMS value of the sum of all harmonic signals divided by the RMS value of the fundamental, expressed as a percentage. This value represents the combined effect of all harmonics in the system.

- An interruption occurs when voltage is completely cut off from a system for some period of time. The length of an interruption is usually described by one of the following terms:

Momentary interruptions last no more than a few seconds, and usually result from load switching.

Temporary interruptions last up to a few minutes. The cause is typically found in the grid, but power can be quickly rerouted around the problem.

Sustained interruptions refer to any loss of voltage for an indefinitely long period of time. Such a condition usually requires some form of manual repair.

- Transients are often called spikes because they occur over a very short period of time, on the order of microseconds. A transient has a distinct beginning and end, and may be further classified as one of the following: 
Impulsive - the voltage returns to its normal value following the transient.

Oscillatory - the transient changes direction and reduces in magnitude repeatedly.

\subsubsection{Effects of Poor Power Quality}

Many electronic devices are connected to the power grid via a DC power supply. Because it is the connection point, it is the component most often damaged by poor power quality. Voltage sags cause increased capacitor charge and discharge currents, which result in excessive heating of the power supply. Periods of voltage over $110 \%$ of nominal can damage rectifiers, switching transistors, and regulators [6].

If a nonlinear load creates distortion in the current waveform, it will also distort the voltage waveform due to the relationship between voltage and current. Voltage harmonics have a direct damaging effect on loads, and will affect all loads connected to the bus where distortion is present. Current harmonics can produce overheating in transformers, abnormally high neutral currents, and overvoltage conditions due to resonance [7].

\subsection{Research Goals}

The overall goal of this research project was to demonstrate the feasibility of a power electronics device that both reduces the power consumed by an induction motor under light load conditions while keeping harmonic disortion at a minimum. In addition, the control algorithm was to be designed for implementation on a single-chip microcontroller. To this end, the following tasks were undertaken:

- Verify the power regulating ability of a triac-based motor controller by constructing a hardware prototype. This controller is to be modeled after an existing design and tested under various load conditions.

- Analyze the harmonic distortion created by the motor controller. Compare test results from running the motor load with the controller with data collected without regulation.

- Create a simulation model of the motor controller for additional testing. Verify the correctness of the model by comparing test results to empirical data. 
- Create a simulation model of a power quality conditioner. An existing analog-based design is to be used as a starting point. Verify the harmonic filtering capabilities of the model by testing with a switching load.

- Alter the power quality conditioner model to change the control scheme from analog to digital. Design the digital system such that it is easily implementable on a microcontroller. Verify the model by comparing test results with data collected from the analog control simulation.

- Combine the motor controller and line conditioner simulation models, and compare test results with data collected from the motor controller simulation without conditioning.

\subsection{Thesis Organization}

This introductory chapter has provided a brief overview of some of the concepts explored by this project. Chapter Two contains a survey of available literature pertaining to this research. From there, specific details of the work performed are provided.

Chapter Three focuses on the motor control aspect of the project. The analysis of an existing design and discussion of operating concepts is followed by details of a hardware implementation. Software written for the microcontroller is also described. Results of testing the prototype are presented, then a Simulink model of the subsystem is discussed. Finally, simulated results are given and compared to the empirical data.

The second subsystem, the line conditioner, is the subject of Chapter Four. Again, an existing design is presented and a Simulink model is described. Modifications made to this design in order to implement the control algorithm on a microcontroller are discussed, followed by simulation results and a comparison of the original analog control system to the digital one. A Simulink model of the integrated motor controller and line conditioner is shown, and results of the complete system simulation are presented and analyzed.

Chapter Five contains a summary and analysis of the results. The overall success of the project is considered, and potential future applications and improvements are discussed. In addition, this chapter contains a reflection on the lessons learned as a result of this research. 


\section{Chapter 2}

\section{Literature Survey}

This chapter presents various literature on subjects related to the research performed during this thesis project. First, a commercially available product line is described that claims to reduce power consumption in induction motor applications ranging from household appliances to industrial processes. Further examples of microcontroller-based motor control applications are presented, illustrating the power and flexibility of such processors. Next, a discussion of harmonic filtering techniques covers passive filters, active filters, and Flexible AC Transmission System (FACTS) devices.

\subsection{Motor Control}

\subsubsection{EnergySmart Power Planner}

Power Planner, by EnergySmart, is a family of devices designed to reduce energy consumption by induction motors. The Power Planner I unit, shown in Figure 2.1, is designed for residential appliances containing a single-phase motor; appliances may be plugged into an outlet on the device. The Power Planner II is also designed for single-phase applications, but must be hardwired to the machine. For three-phase motor applications such as those used in heavy industrial environments, a Power Planner III unit may be employed. Each device utilizes an 8-bit RISC (Reduced Instruction Set Computer) microcontroller and proprietary software algorithms [8].

EnergySmart claims that the Power Planner can decrease energy consumption by as much as $52.7 \%$, leading to a significant reduction in operating costs. In addition, the operating temperature of the motor is reduced, prolonging the life of the equipment. The Power Planner is 


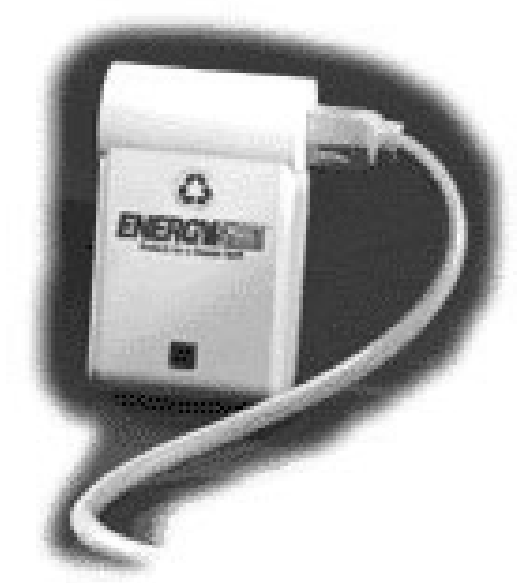

Figure 2.1: EnergySmart Power Planner I (From [8])

versatile, as it can operate at a variety of voltage levels at both $50 \mathrm{~Hz}$ and $60 \mathrm{~Hz}$ frequencies [8].

It was found that the Power Planner controls the energy delivered to the load using a triac in series with each phase winding of the motor. The microcontroller outputs a PWM (Pulse-Width Modulated) control signal to the gate of the triac, varying the duty cycle of the applied power based on the magnitude of the load [9].

The Power Planner determines the power factor, and thus the motor load, using only the zero-crossings of the voltage and current waveforms. A specialized subcircuit is used to detect when the voltage crosses zero. The zero-crossing of the load current can be determined by the voltage level at the triac gate. These signals are input to the microcontroller, which produces an output such that when the amount of power applied to the load is excessive, the voltage is cut off. The length of the cutoff period is proportional to the load magnitude; the voltage cutoff period will be longer under light load conditions and shorter with a heavy load. However, due to intertia the speed and torque of the rotor are unaffected [9].

The Power Planner circuit is protected by United States Patent 5,592,062, issued to Daniel G. Bach. The contents of this document are discussed further in Section 3.1.

\subsubsection{Other Motor Controllers}

Various motor control applications utilize the computational power of modern microcontrollers to achieve highly accurate control while reducing the number of circuit components. A few examples of such designs are presented in this subsection. 


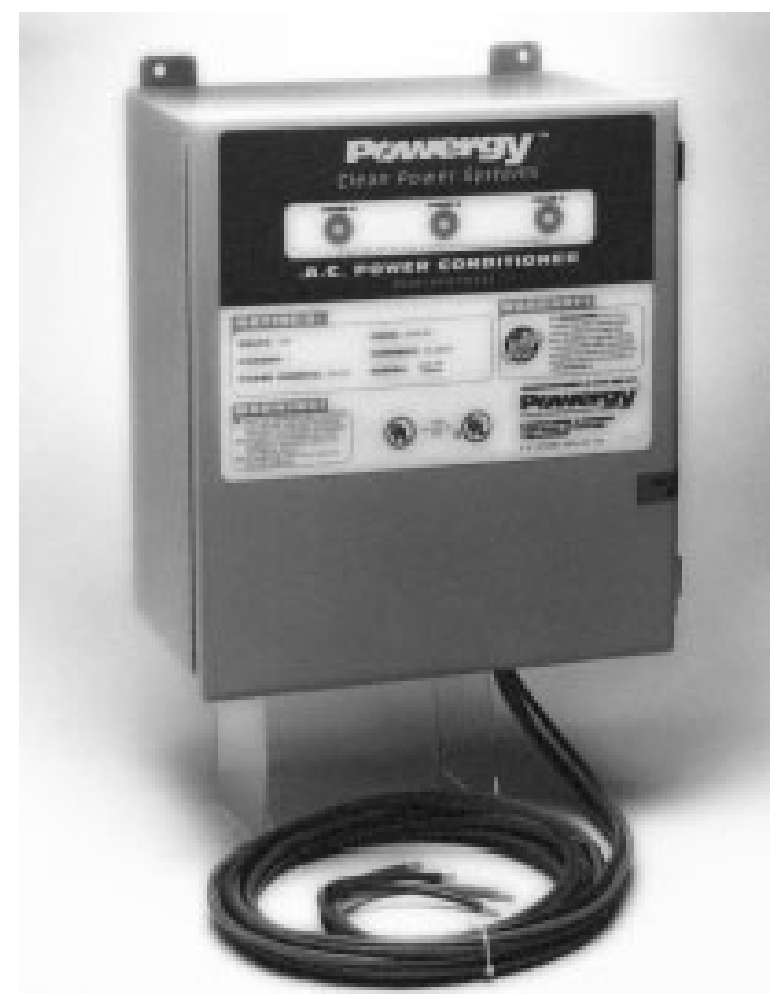

Figure 2.2: Powergy Clean Power Systems Unit (From [10])

Powergy, Inc. of Providence, RI, produces a device called the Clean Power Systems unit, shown in Figure 2.2. The CPS corrects power factor, filters noise, and provides protection against surges and spikes. Usage ratings range from $120 \mathrm{~V}$ single-phase to $600 \mathrm{~V}$ three-phase. The Motor Systems Resource Facility at Oregon State University tested the CPS on a 100 horsepower motor, and the results showed power factor and current improvement at both full and partial load. However, while power consumption was reduced at full load, it increased slightly at partial load [10].

In [11, a speed control system for a three-phase induction motor is described. This application utilized Hall effect sensors on the motor shaft to detect the speed of the motor, which is an indicator of the load. A control system was needed to filter signals from the sensors and detect unusually large load changes. The controller was modeled as a finite state machine, then implemented on an Atmel 89C2051 microcontroller.

The authors justify the use of a microcontroller for several reasons, among them being cost, flexibility, and the ease of implementing the finite state machine control system in software. Ben- 
eficial features of the processor are listed, though the authors acknowledge a limitation imposed by the amount of available RAM on the chip. Due to the complexity of filtering techniques under consideration, a normalization scheme was developed to allow the memory-intensive routines to function with the 89C2051's 128 bytes of RAM [11].

The importance of efficient motor operation has been recognized by manufacturers of microcontrollers and digital signal processors. Microchip, maker of the PIC microcontroller, has released the dsPIC30F product line, which combines the functionality of a 16-bit microcontroller with the processing power of a DSP. A subset of this product family is specially designed for motor control applications, and includes features such as simultaneous analog-to-digial conversion and multiple pulse-width modulation options [12]. Other chip makers, such as Atmel and Freescale, also have specialized motor control processors.

An application of these new specialized processors is presented in [13]. A dsPIC30F2010 microcontroller is used to implement a boost-buck power factor correction system. This system samples a rectified AC line voltage at a high frequency and uses a switching MOSFET to produce a DC voltage supply in such a way that it draws an average current that appears resistive to the AC source. It is claimed that the control algorithm presented is infeasible to implement using analog techniques.

Op-amp circuits are used to scale down the input voltages to the microcontroller, which include the rectified AC voltage, the raw DC voltage created from the boost converter, and the output DC voltage that has been filtered and regulated. The scaled-down signals are input to the analog-to-digital converter of the dsPIC. Output signals are created by the processor's built-in pulse-width modulation hardware, with software computing the appropriate parameters of the pulses on the fly. Results shown indicate a sizeable improvement over standard rectified DC power supplies [13].

\subsection{Harmonic Filters}

\subsubsection{Passive Filters}

Because harmonics are waveforms at multiples of the fundamental frequency, low-pass or bandpass filters can be used to reduce distortion. However, these passive filters only attenuate the unwanted frequencies rather than eliminate them, and usually cause a reduction in the amplitude of the source signal. The simplest low-pass filters consist of a resistor in series with either a 


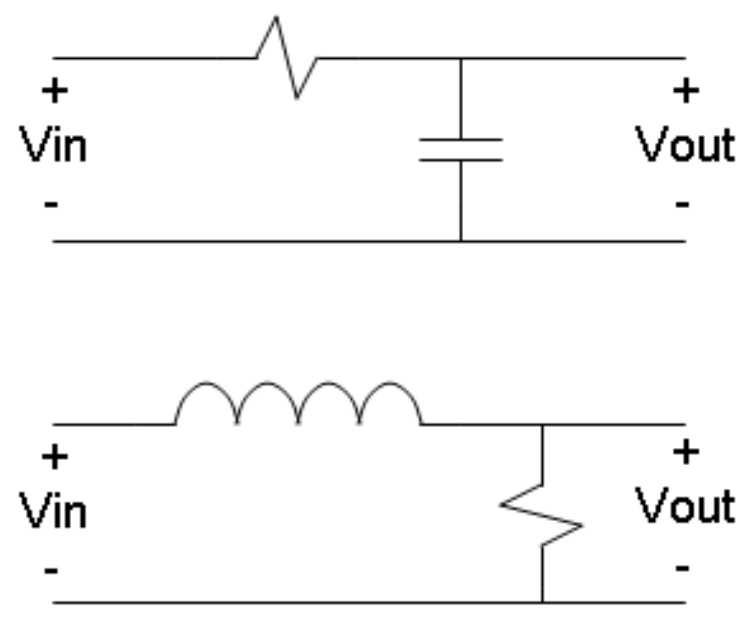

Figure 2.3: Low-Pass Filter Circuit Diagrams

capacitor or inductor, and the values of the circuit elements are chosen based upon the cutoff frequency. Example circuit diagrams are shown in Figure 2.3. Most often, the cutoff point is chosen to be the frequency that delivers half of the maximum average signal power. Low-pass filters are so named because they allow frequencies below the cutoff frequency to pass through [3].

Bandpass filters are slightly more complex, and are defined by a center frequency and a bandwidth. These circuits usually contain both capacitors and inductors, the values of which are selected such that their impedances are of equal magnitude and opposite sign at the center frequency. Due to the reactive elements that make up a bandpass filter, the phase angle of the input signal will be affected except at the center frequency [3].

\subsubsection{Active Filters}

Active filters generate harmonic currents using power electronics devices, and when these harmonics are added to the AC line they cancel out existing distortion. These filters may be connected in series or in parallel with the AC source; the type of connection determines how components must be sized. Series filters are able to remove distortion caused by the connected load as well as harmonics already present in the AC line. Hybrid solutions that combine active and passive filters are also used [14. 


\subsubsection{FACTS Devices}

Flexible AC Transmission System (FACTS) devices are power electronic systems used for controlling power transmission parameters, such as impedance, current, voltage, and phase angle. Such controllers utilize high-power transistors, or thyristors. Series FACTS controllers inject voltage to the line, while shunt controllers inject current. Both types will only source or sink reactive power as long as the injected signals are in phase with the line signals. There are also combined series-shunt FACTS controllers that regulate reactive power on individual lines while allowing real power to transfer among the lines [15].

The Statcom, or Static Compensator, is a shunt FACTS controller that is primarily used for controlling reactive power independent of the system voltage [15]. By utilizing high-speed IGBTs, Statcom devices can produce high-frequency signals to cancel harmonics. This inherent active filtering eliminates the need for external harmonic filters, which results in a lesser risk of resonance [16].

A Personal Static VAR Compensator (PSVC) is discussed in [17], and provides a good example of a microcontroller-based FACTS device. The design is a variation on the standard SVC, using both capacitors and an inductor to adjust the power factor of a reactive load. To detect the power factor of the load being controlled, a voltage divider and current transformer are used to capture the associated waveforms, which are filtered and passed through voltage comparators to a PIC microcontroller. Use of the comparator chips allows digital input pins to be used so the voltage and current states can be polled nearly simultaneously.

Inputs to the PIC are used to detect the voltage and current zero-crossings, and based on the timing between them the power factor is calculated and classified as leading or lagging. With this information the microcontroller software, which includes a fuzzy logic algorithm, can determine which capacitors to switch on and the required firing angle of the inductor. Control pulses are generated by the PIC to activate thyristors connected to the compensating elements. Although the PSVC was shown to improve power factor and reduce current draw, the total harmonic distortion of the load current increased significantly [17].

\subsection{Thesis Objective}

The objective of this thesis is to combine a power-saving motor control system with a line conditioner to reduce harmonic distortion induced by power electronics elements. The system 
should be implementable on a single, low-cost, commercially available microcontroller with as few support components as possible. A hardware implementation and test of the motor control subsystem will be performed to ensure understanding of its operation and the associated harmonic distortion problem. Data collected from the hardware test will be used to verify a Simulink model of the subsystem. Simulation of an active line conditioner will also be performed, then the design of the harmonics-filtering controller will be altered to reflect the software environment of a PIC microcontroller. After simulating the digital control algorithm and ensuring its correct operation, the two subsystems will be combined and simulated. 


\section{Chapter 3}

\section{Motor Controller}

Although an existing design was used as the basis for the motor control subcircuit, the implementation was challenging due to the lack of a detailed description of the software algorithm. This chapter begins with an analysis of the circuit diagram, followed by algorithm development, software design, implementation, and testing. Experimental test results are then presented that show a significant reduction in consumed power when using the controller, especially under light load conditions. Finally, a simulation model of the motor controller is presented and discussed.

\subsection{Power Planner Patent}

\subsubsection{Overview}

The circuit diagram from United States Patent 5,592,062 is shown in Figure 3.1. While the schematic does not contain a large number of components, it is partitioned into four distinct subcircuits that perform specialized functions. A brief description of each subcircuit is as follows [18]:

1000 is a microcontroller. It is shown with two analog inputs RA2 and RA3, one digital output RB0, power input VDD, and reset pin MCLR. The patent document goes on to indicate that the microcontroller contains an analog-to-digital converter, processor, and memory.

2000 is an inverting amplifier that serves to detect zero-crossings in the voltage waveform. Although no arrow appears on transistor 130, it is assumed to be NPN by the configuration. 3000 is a DC power supply, used to provide the microcontroller and other DC components with 


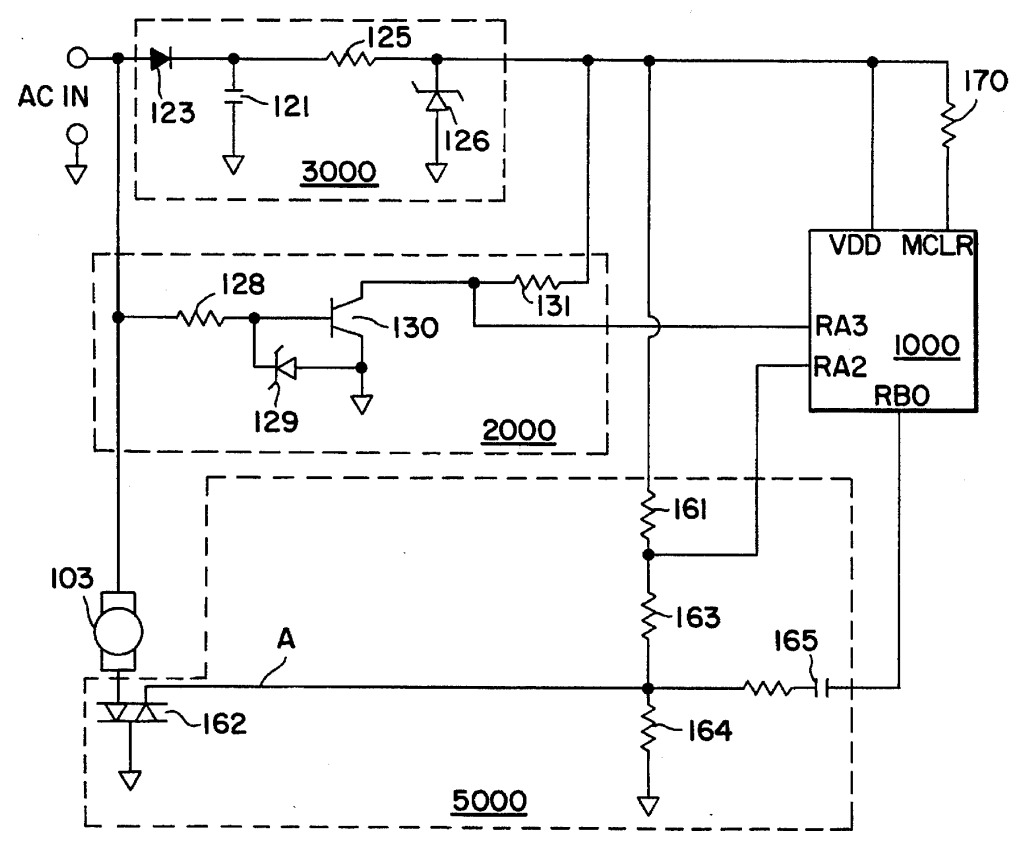

Figure 3.1: Bach's Circuit Diagram (From [18])

a constant five volts.

5000 includes the triac and a voltage divider circuit that serves as an interface to the microcontroller. It should be noted that the symbol for triac 162 appears to be upside-down.

\subsubsection{Analysis}

The voltage zero-crossing detection subcircuit (2000) produces a square wave that changes state when the signal at the base of the transistor changes sign. When the AC voltage is below a small threshold voltage (usually less than one volt), transistor 130 is cutoff and no current passes through resistor 131 . Thus, the voltage at microcontroller pin RA3 is equal to the DC source voltage of $+5 \mathrm{~V}$. Once the threshold voltage is exceeded, the transistor operates in the active mode for a short time, during which the voltage at the collector drops sharply. The transistor then becomes saturated as the $\mathrm{AC}$ voltage continues to rise, and the resulting input on pin $\mathrm{RA} 3$ is close to zero volts [19]. The waveform is illustrated in Figure 3.2 .

The gate voltage of a triac is determined by the current passing through it, unless being driven to a specific value by some external means. When in the nonconducting state, the gate voltage is zero; when conducting, the gate voltage may be positive or negative, depending on the 


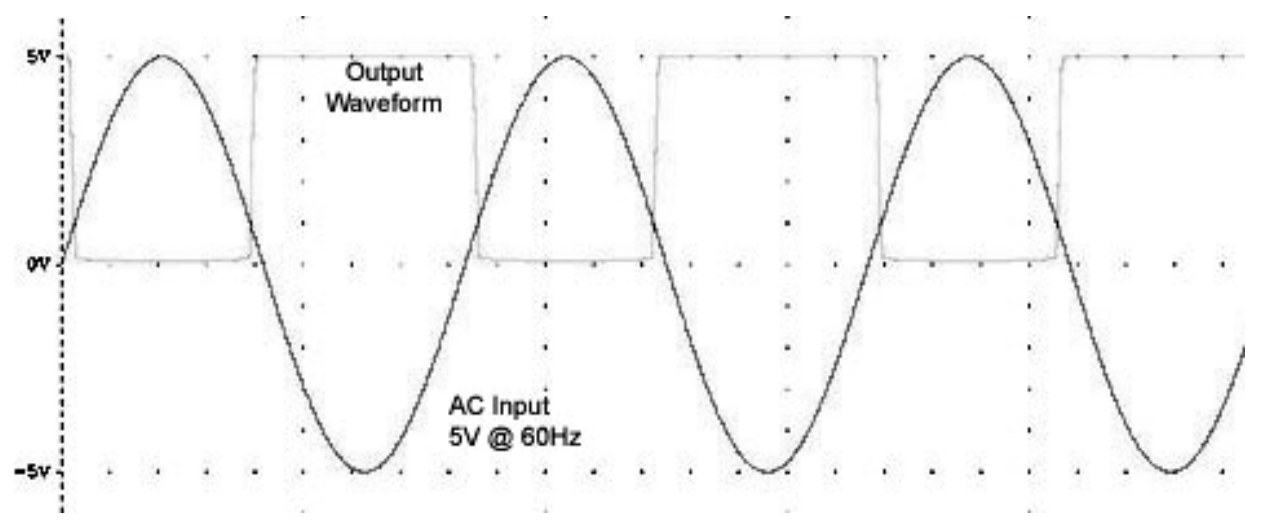

Figure 3.2: Inverting Amplifier Waveform

sign of the current waveform. The magnitude of the gate voltage while in the conducting state is device-dependent, as is the magnitude of the signal voltage required to turn the triac on. When the current becomes zero the triac reverts to its nonconducting state automatically, unless the gate voltage is driven to exceed the on-signal threshold [20].

In order for the microcontroller to detect zero-crossings in the current waveform, it must observe the behavior of the triac gate voltage. However, the analog-to-digital converters found in microcontrollers typically read from $0 \mathrm{~V}$ to some positive DC reference voltage; to accept negative voltage levels, other reference voltages must be supplied as inputs to the ADC. To avoid adding complexity to the circuit, the triac gate voltage, indicated by signal A in Figure 3.1, is pulled up so that it falls between $0 \mathrm{~V}$ and $5 \mathrm{~V}$. This is accomplished through the use of the voltage divider formed by resistors 161, 163, and 164 .

To determine the voltage at pin RA2, the principle of superposition is applied. This is necessary because there are two sources: the DC voltage $V_{D D}$ and the triac gate voltage $V_{g}$. When $V_{g}$ is at $0 \mathrm{~V}$, pin RA2 sees $V_{a}$ as given in Equation 3.1. Likewise, when $V_{D D}$ is removed the output voltage is given by Equation 3.2. The actual voltage at pin RA2 is the sum of $V_{a}$ and $V_{b}$, as shown in Equation 3.3 . 


$$
\begin{aligned}
V_{a} & =V_{D D} * \frac{R_{163}}{R_{161}+R_{163}} \\
V_{b} & =V_{g} * \frac{R_{161}}{R_{161}+R_{163}} \\
V_{R A 2} & =V_{a}+V_{b} \\
& =\frac{V_{D D} * R_{163}+V_{g} * R_{161}}{R_{161}+R_{163}}
\end{aligned}
$$

Using the input on pin RA3, the microcontroller can determine when the voltage applied to the motor has crossed zero. Likewise, the signal at pin RA2 determines when the current has crossed zero. The time difference between the zero-crossings indicates the phase angle - and thus the power factor - of the load, and the microcontroller adjusts the triac firing delay accordingly. The triac is turned on by a pulse from pin RB0 [18].

Software stored in the microcontroller's memory is used to calculate the power factor and determine when to signal the triac to conduct. Details of the algorithms used for these purposes are difficult to determine from the patent description; however, several specific features are mentioned [18]:

- The system determines the frequency of the voltage waveform using the first few pulses on pin RA3, allowing operation at 50 and $60 \mathrm{~Hz}$.

- If a resistive or very heavy load is detected, the duty cycle is set to $100 \%$, passing the full power from the AC source to the load.

- A soft start is used to gradually bring the motor to full power.

- When the phase angle of the load changes, the duty cycle is adjusted over time rather than instantaneously.

\subsection{Hardware Implementation}

A hardware prototype was constructed and tested to further study the behavior of the motor controller system. The circuit diagram shown in Figure 3.3 differs slightly from Figure 3.1, as a MOC3051 triac driver [22] and an RC filter have been added. Note that Figure 3.3 does not include any LEDs or pushbuttons that may have been added during intermediate testing.

To reduce complexity during testing, a stand-alone DC power supply was used instead of rectifying the $\mathrm{AC}$ voltage. Because this equipment was used only for the purpose of supplying 


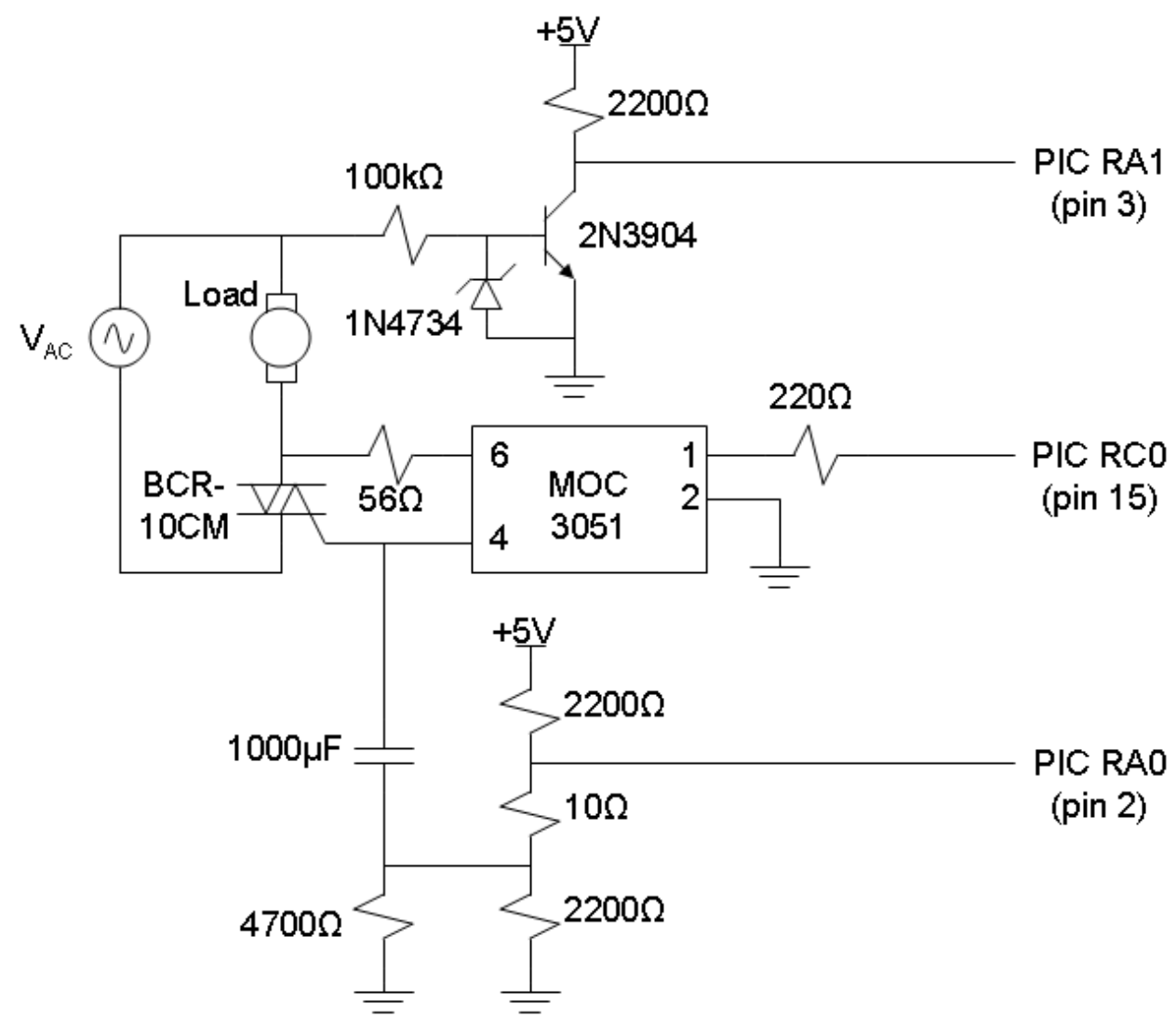

Figure 3.3: Motor Controller Circuit Diagram

$+5 \mathrm{~V} \mathrm{DC}$ to the circuit, it had no functional effect on the controller. When mixing AC and DC signals, the AC neutral was tied to the negative terminal of the DC supply.

The Microchip PIC18F452 was chosen as the microcontroller for this project. It is a very versatile chip, containing a 10-bit analog-to-digital converter (ADC), pulse-width modulation (PWM), high- and low-priority interrupt vectors, several timers, and many more features [21].

Load control is performed using a BCR10CM triac, which is rated for $10 \mathrm{~A}$ maximum. This triac requires a $1.5 \mathrm{~V}, 30 \mathrm{~mA}$ input signal on the gate to turn on, and can accept up to $10 \mathrm{~V}$ and $2 \mathrm{~A}$ maximum. It is intended for use in household equipment, including small motor applications [23].

A Yokogawa PZ4000 power analyzer was used to gather measurements while testing the motor controller circuit. This unit has four slots for plug-in input modules that can accept up to $1000 \mathrm{~V}$ and 20A. A color display provides a user interface and independently-scaled curves on each input channel. For data capture, thermal printouts can be made or the unit can store the information 


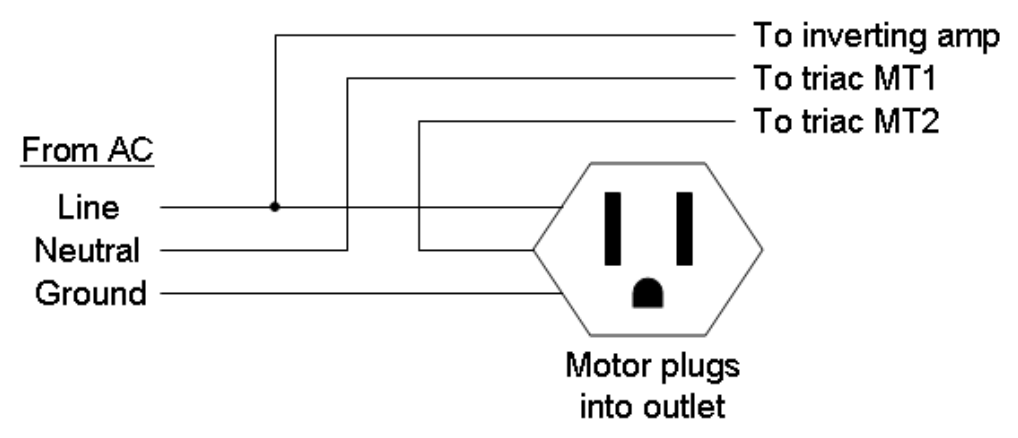

Figure 3.4: 115V Load Outlet Schematic

to computer media using RS-232, SCSI, or a floppy disk [24].

\subsubsection{Connection of the Load}

A custom outlet, with schematic as shown in Figure 3.4, was used to interface the load to the electronics. This outlet was plugged into a standard $115 \mathrm{~V}$ wall socket, and delivered power to the load via an internal connection. Three leads were extended from the custom outlet to the control circuit. The first of these was connected directly to the AC voltage, and served as the input to the inverting amplifier.

The remaining two leads were used to connect the load and the controlling triac. The return line from the load was separated from the wall outlet neutral wire and tied to the MT2 terminal on the triac. The MT1 lead was then connected to the AC neutral, completing the circuit.

\subsubsection{Testing Setup}

For testing, the motor controller was connected to a single-phase induction motor with specifications as shown in Table 3.1. To vary the load on the motor, a leather belt was wrapped around the rotor. One end of the belt was fastened to a large plate of metal, as was the motor. The other end of the belt was connected to a metal block with a threaded hole all the way through. This block was situated on a shelf with an upward-protruding side. A bolt was run through the protrusion and block: this bolt could then be turned to tighten or loosen the belt.

Initially, the amount of load on the motor was determined by measuring the distance from the end of the shelf to the block. However, after several test runs the belt began to wear down from heat and stress, so this metric could not be considered consistent. Instead, a modification was made to include a small scale between the block and belt. The setup is shown in Figure 3.5 . 


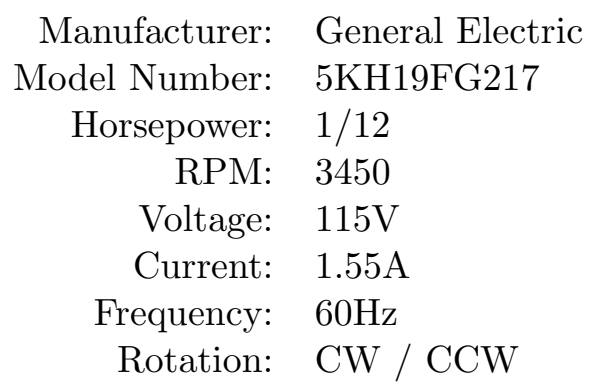

Table 3.1: Test Motor Specifications

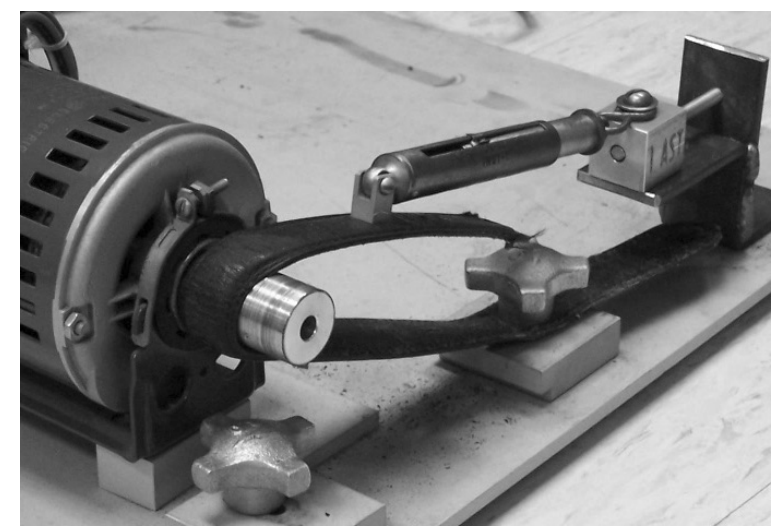

Figure 3.5: Photograph of Test Load Setup

By using a scale attached to the belt, it was possible to describe the motor load in terms of torque. The rotor measures one inch in diameter; therefore, multiplying the scale reading (in ounces) by 0.5 gives the torque applied to the center of the rotor in inch-ounces.

\subsection{Software Design}

A high-level software system diagram is shown in Figure 3.6. The PIC I/O system receives signals from the triac gate pull-up and the inverting amplifier. These are converted to states and passed to the zero-crossing detector. The time between zero-crossings is then used to calculate the time delay for the triac control pulse. This pulse is generated by the I/O block and sent to the MOC3051 triac driver. For a complete listing of the dutyControl.c source file, see Section A.1. 


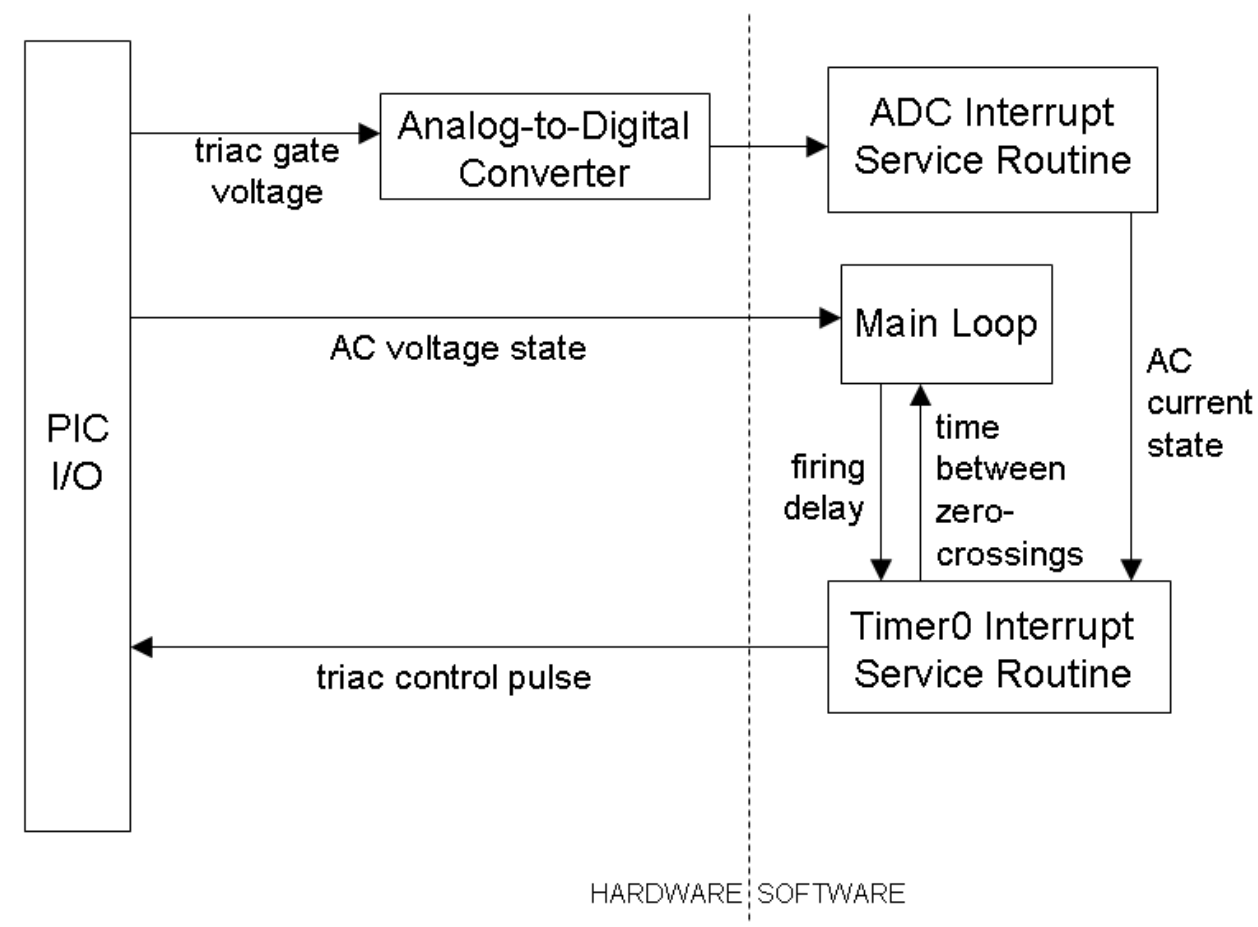

Figure 3.6: Motor Control Software Block Diagram

\subsubsection{Zero-Crossing Detection}

As previously stated, there are two types of zero-crossings to detect: voltage and current. Voltage zero-crossings are detected by watching the output of the inverting amplifier, while the pulled-up triac gate voltage is used to detect current zero-crossings. According to [18, both signals are fed into the ADC of the microcontroller.

In the initial code, the ADC was read during an interrupt service routine (ISR) that was executed based on the PIC's internal Timer0 module. Reading both channels required interrupts to be scheduled every $100 \mu \mathrm{s}$, even with a clock speed of $20 \mathrm{MHz}$. Much of the time required for analog-to-digital conversions is spent on channel acquisition, so the ISR was re-coded to alternate reads between the two channels. After reading, the acquisition was initialized and the ISR terminated, allowing time for calculations in the main loop.

A voltage zero-crossing occurs when the output of the inverting amplifier changes from near $5 \mathrm{~V}$ to near $0 \mathrm{~V}$, or from near $0 \mathrm{~V}$ to near $5 \mathrm{~V}$. Although there is a small transition time between the saturation and cutoff states of the transistor in the inverting amplifier, it is negligible and not important for detecting zero-crossings. Therefore, in the final implementation the collector 
voltage was connected to a digital input port on the PIC rather than an analog one, eliminating the need for channel acquisition.

Because the current zero-crossing detector was required to differentiate among three states of the triac gate voltage, using a digital input was not possible. The three states were designated as nonconducting, positive half-wave, and negative half-wave. The Yokogawa power analyzer 24] was used to find boundaries in the pulled-up gate voltage waveform. Then, these values were compared against the analog input to determine the state of the current waveform. Any transition from conducting to nonconducting was considered a zero-crossing; however, transitions from nonconducting to conducting were ignored because they were caused by the PIC-generated control pulse.

The PIC18F452 supports an ADC interrupt, so that a conversion may be started and other work may be performed instead of polling the ADC until the operation is complete. Taking advantage of this feature greatly improved performance. See Section 3.3 .3 for more information.

\subsubsection{Duty Cycle Adjustment}

The phase angle of the load can be determined by counting the number of timer interrupts between the voltage and current zero-crossings. For the most robust system, an initialization step is necessary to detect the line frequency [18]. Rather than store this information as a frequency, however, a variable is set to the number of interrupts executed during a half-wave of the voltage waveform.

Duty cycle adjustment occurs in the main loop of the software, shown as a flowchart in Figure 3.7. When a voltage zero-crossing occurs, a counter variable is reset. This counter is incremented by one during the Timer0 ISR, which is executed at fixed $30 \mu$ s intervals. When the current crosses zero, the counter is checked against the number of interrupts in a half-wave. It is important to note that only resistive and inductive loads were taken into account: this eliminated the need to check the signs of the current and voltage half-waves at zero-crossings.

Once the time between zero-crossings was established, it was converted to a phase angle measured in degrees. If the phase angle was less than $15^{\circ}$ or greater than $90^{\circ}$, the load was considered to be resistive and a flag was set. Otherwise, the firing delay was adjusted by a small increment.

Making small adjustments to the duty cycle proved to be more practical than calculating an "optimal" duty for the detected phase angle. While a few different formulas were tried during 


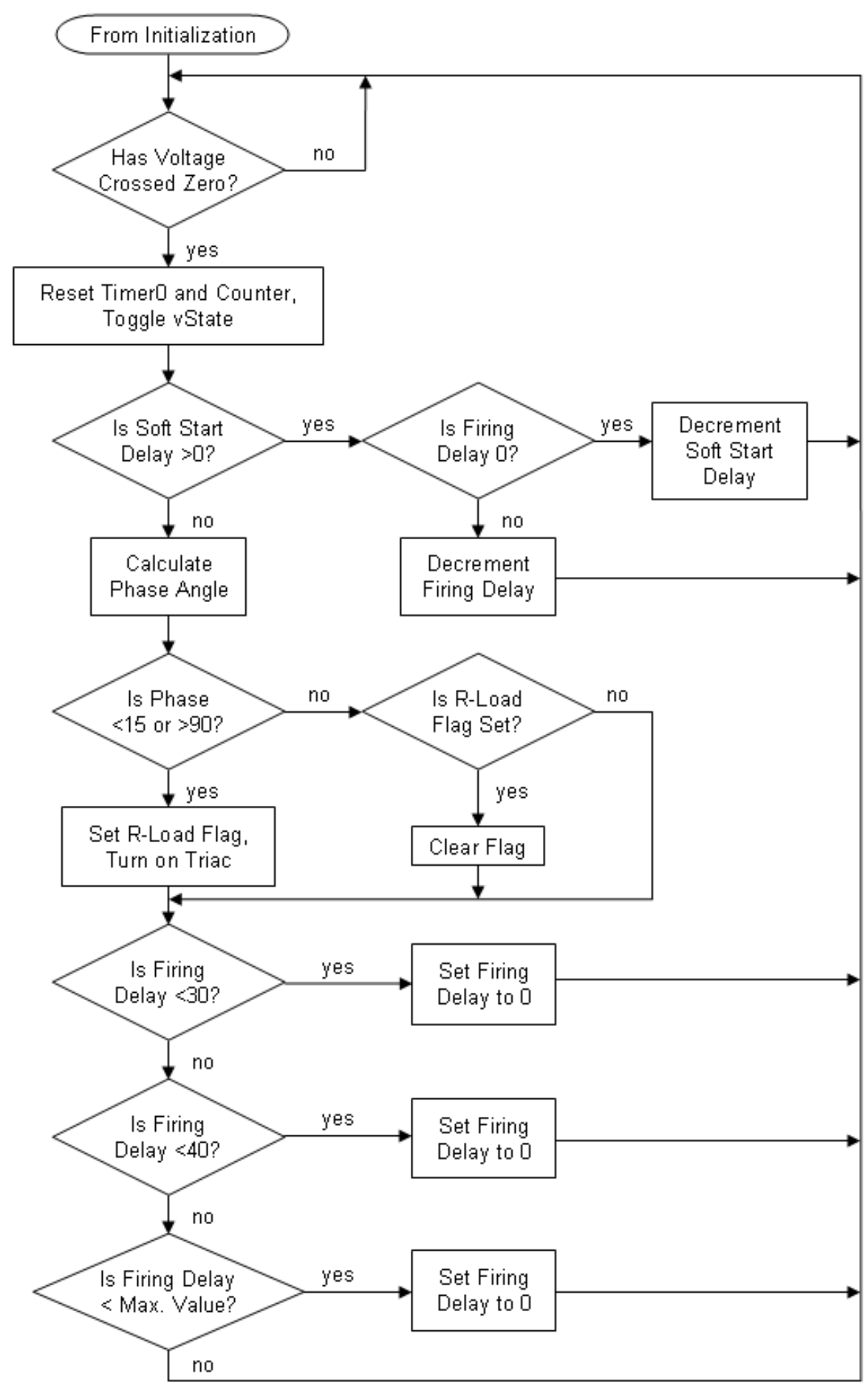

Figure 3.7: Motor Controller PIC Software Main Loop 
experimentation, none of them could be proven to provide the best firing delay for all loads. Those algorithms also required additional computational cycles as well as logical code to change the duty gradually.

\subsubsection{Interrupts}

The PIC18F452 has two interrupt vectors, classified as low- and high-priority. High priority interrupts can occur even when low priority ISRs are executing. This is a very valuable feature and was used in this project for precision timing.

Two ISRs were needed: one for timing and another for reading and restarting the ADC. The timing interrupt was based on Timer0, and was set as a high-priority interrupt scheduled at $30 \mu \mathrm{s}$ intervals. Because it was the only high-priority interrupt, the Timer0 ISR was guaranteed to occur on time, and thus was used as a timing basis for other functions.

When the Timer0 module rolls over from 65535 to 0 , it generates an interrupt and executes the routine shown in Figure 3.8. The first line of the ISR resets Timer0 so that $30 \mu$ s will pass before the next interrupt. An interrupt counter variable, which serves as a timekeeping basis, is then incremented. Next, the state of the load current is checked to determine if a zero-crossing has occurred, and if so the phase counter and pulse flag are reset. The time since the last current zero-crossing is calculated by subtracting the phase counter from the interrupt counter. If the resistive load flag has been set, the ISR does no more; otherwise the gate control pulse is turned on and off as appropriate.

The ADC interrupt was configured such that the ISR would execute whenever a conversion completed, freeing up computation time that would otherwise be spent polling. When the ISR was called, the ADC value was read and compared to the current state boundaries (see Section 3.3.1 for details). If the value read by the ADC did not fall into one of the predefined states, the current was assumed to be in transition and the state variable was assigned the value of the previous state. Before exiting the ISR, the GO_DONE flag is set to signal the PIC to start a new analog-to-digital conversion. A flowchart for this ISR is shown in Figure 3.9.

\subsubsection{Programming}

Most of the source code was written in C and compiled using the CCS PCW application. PCW includes an IDE, compiler, and debug utilities, and is designed for use with most PIC microcontrollers. Standard C structures, such as if statements and while loops, are supported 


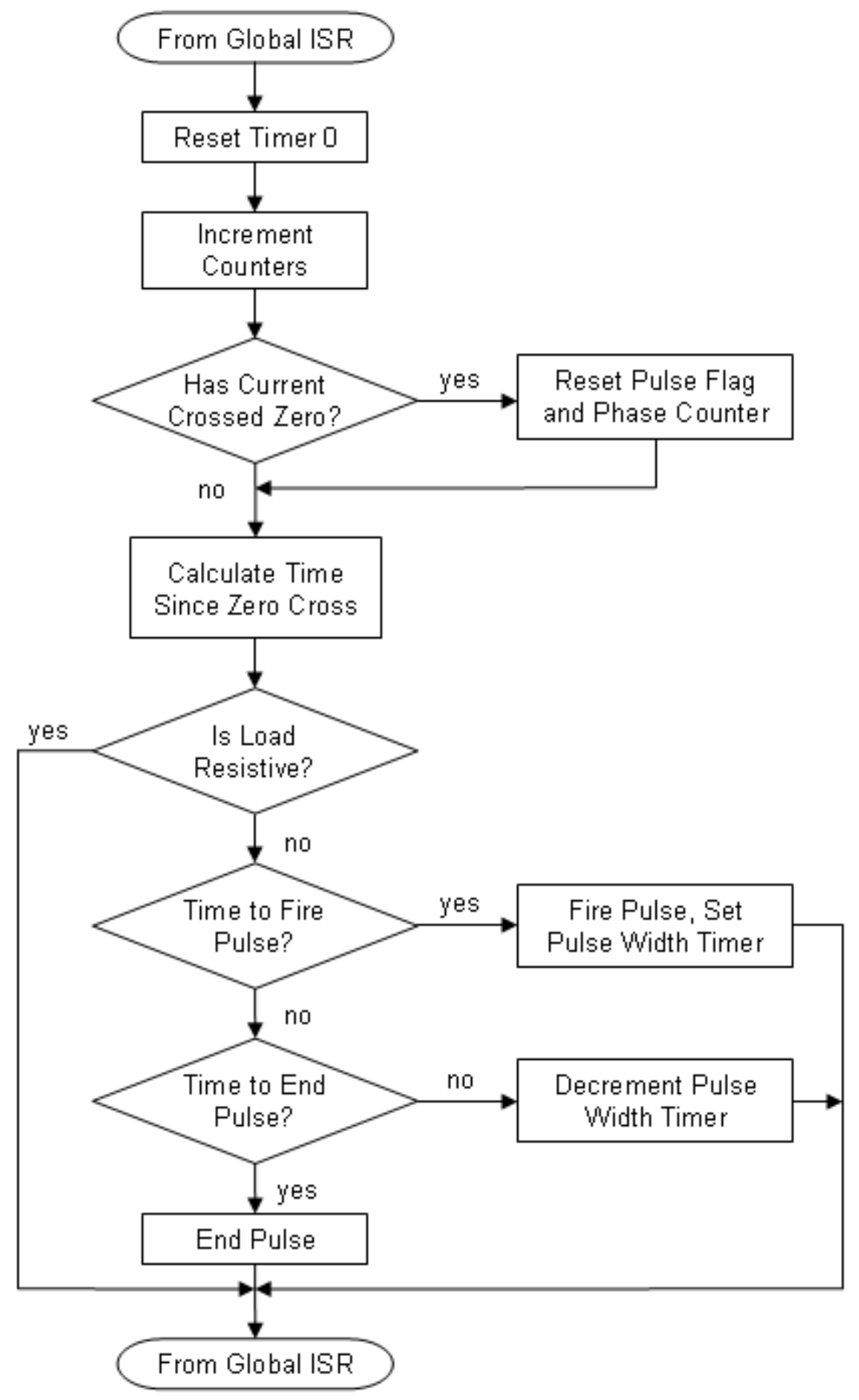

Figure 3.8: PIC Software Timer0 ISR 


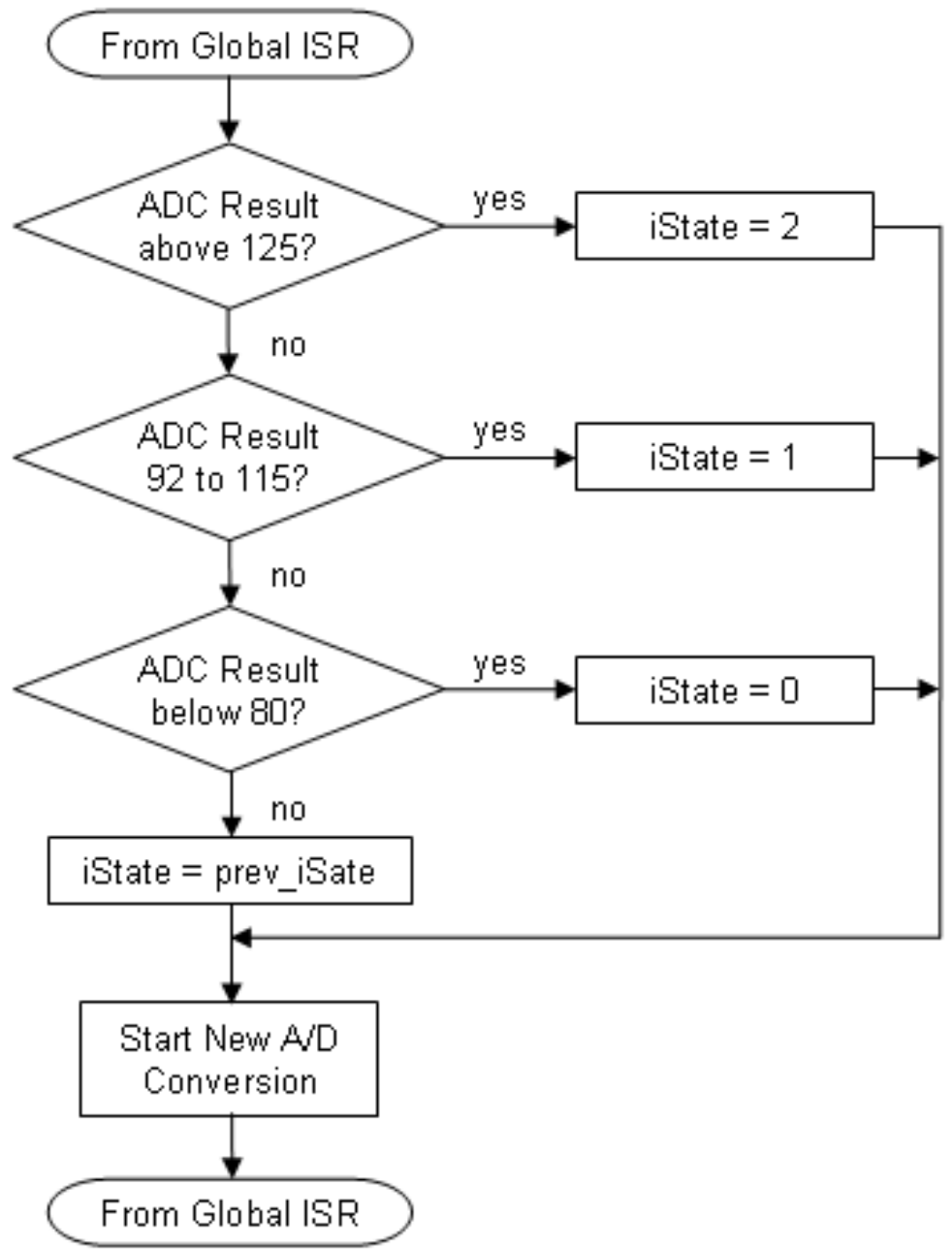

Figure 3.9: PIC Software Analog-to-Digital ISR 
along with PIC-specific calls for enabling/disabling features, reading the ADC, setting timers, and so on [25].

PCW also supports inline assembly code, which was needed to create separate high- and low-priority interrupt handlers. While the compiler is capable of generating ISR functions, it is limited to only one interrupt vector. Therefore, the global interrupt handler was coded in assembly, with individual ISRs coded in C. Assembly was also used in places throughout the program to speed up or optimize certain operations.

A flash memory programmer and header were used to transfer the compiled code from the development workstation to the PIC microcontroller. Once the program was downloaded, the programmer was removed and the circuit was power cycled to begin operation. In-circuit emulation and debugging features were not used.

\subsection{Test Results}

The system was tested with various loads, both with and without the controller; the results are shown in Table 3.2. Selected waveforms from the Yokogawa power analyzer are shown in Figures 3.10 - 3.13. It should be noted that the voltage traces in the scanned printouts were measured across the terminals of the motor and not line-to-neutral; this was done in order to measure the power consumed by the motor, which continues to spin even when the triac is switched off.

It can be readily observed from Table 3.2 that the motor controller successfully reduced the RMS current and total power drawn at all motor load levels. On average, real power consumption was lowered by $23.2 \%$, with a maximum reduction of $56.3 \%(19.24 \mathrm{~W})$ under no load. As expected, the power savings decrease as the motor is more heavily loaded.

To test the controller's ability to detect resistive loads, a $25 \mathrm{~W}$ light bulb was used. When the bulb was the only load, the controller allowed it to run at full duty, as shown by the small differences in values in Table 3.2 and curves in Figure 3.13. However, when the light bulb and motor were used in parallel, some clipping of the input current did occur. This is because the resistance of the light bulb was not high enough to significantly change the phase angle of the load.

While general trends can be discovered in the data, it is important to recognize that some of the measurements do not seem to fit properly. For example, the numbers for the 16oz. load appear inconsistent with the rest of the table, especially the THD figures. As mentioned earlier, 


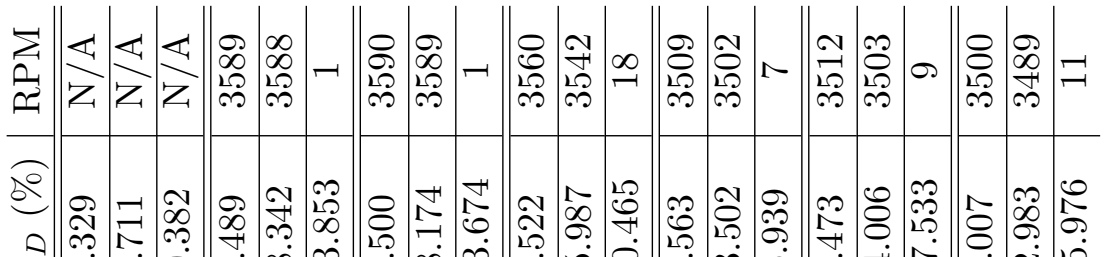

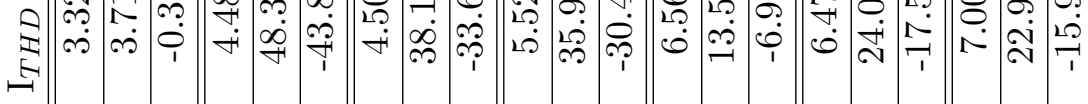

so

a E)

空

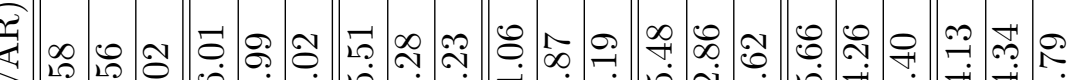
Z

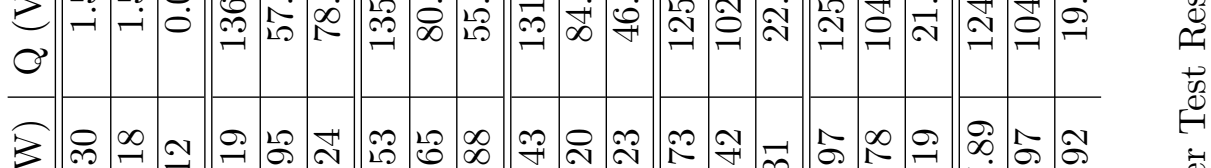

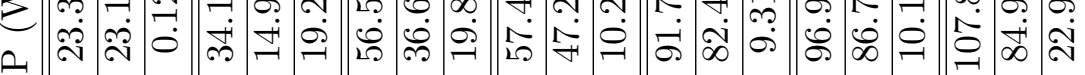

岁的

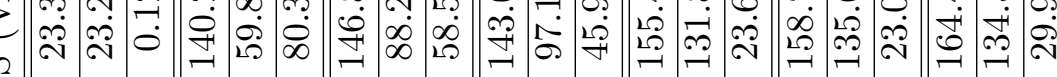

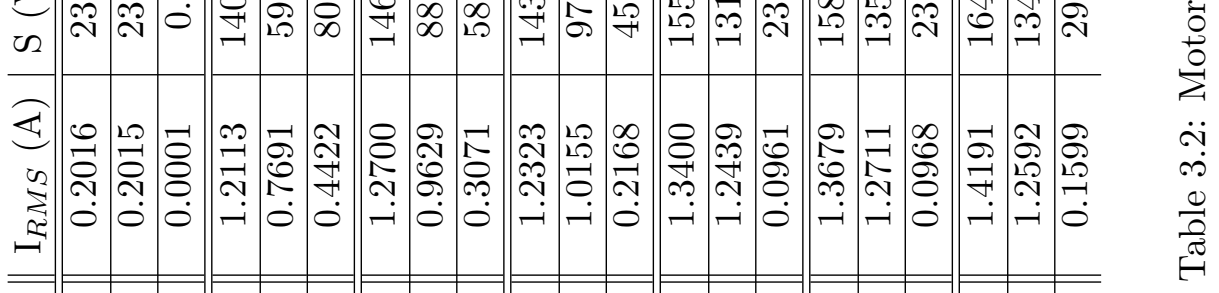

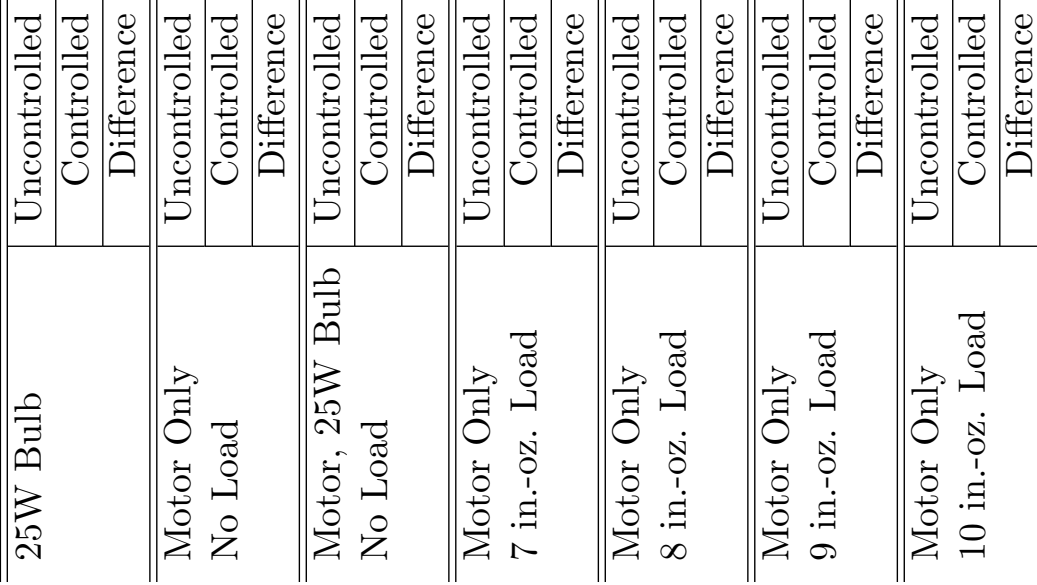




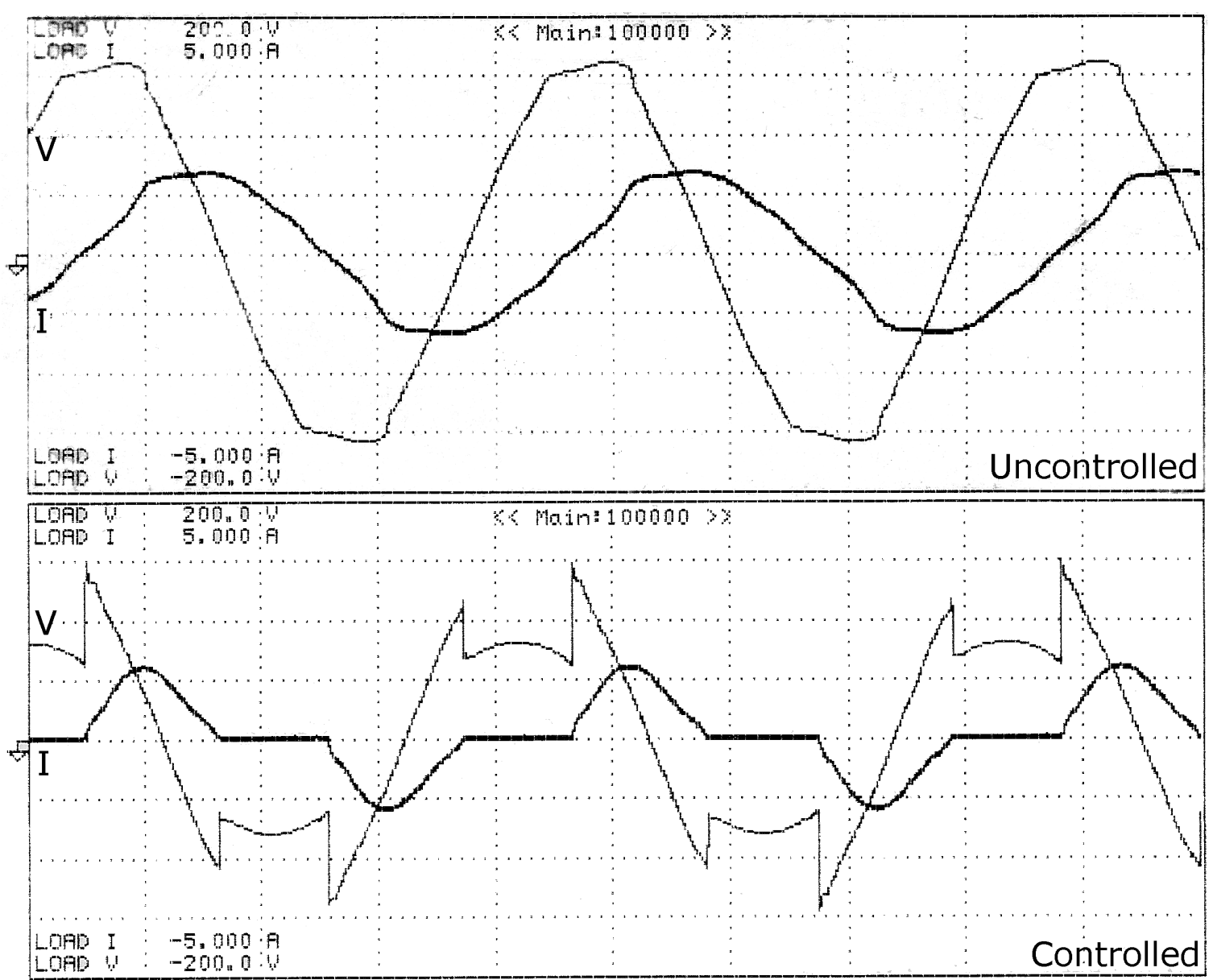

Figure 3.10: Experimental Results, No Load 


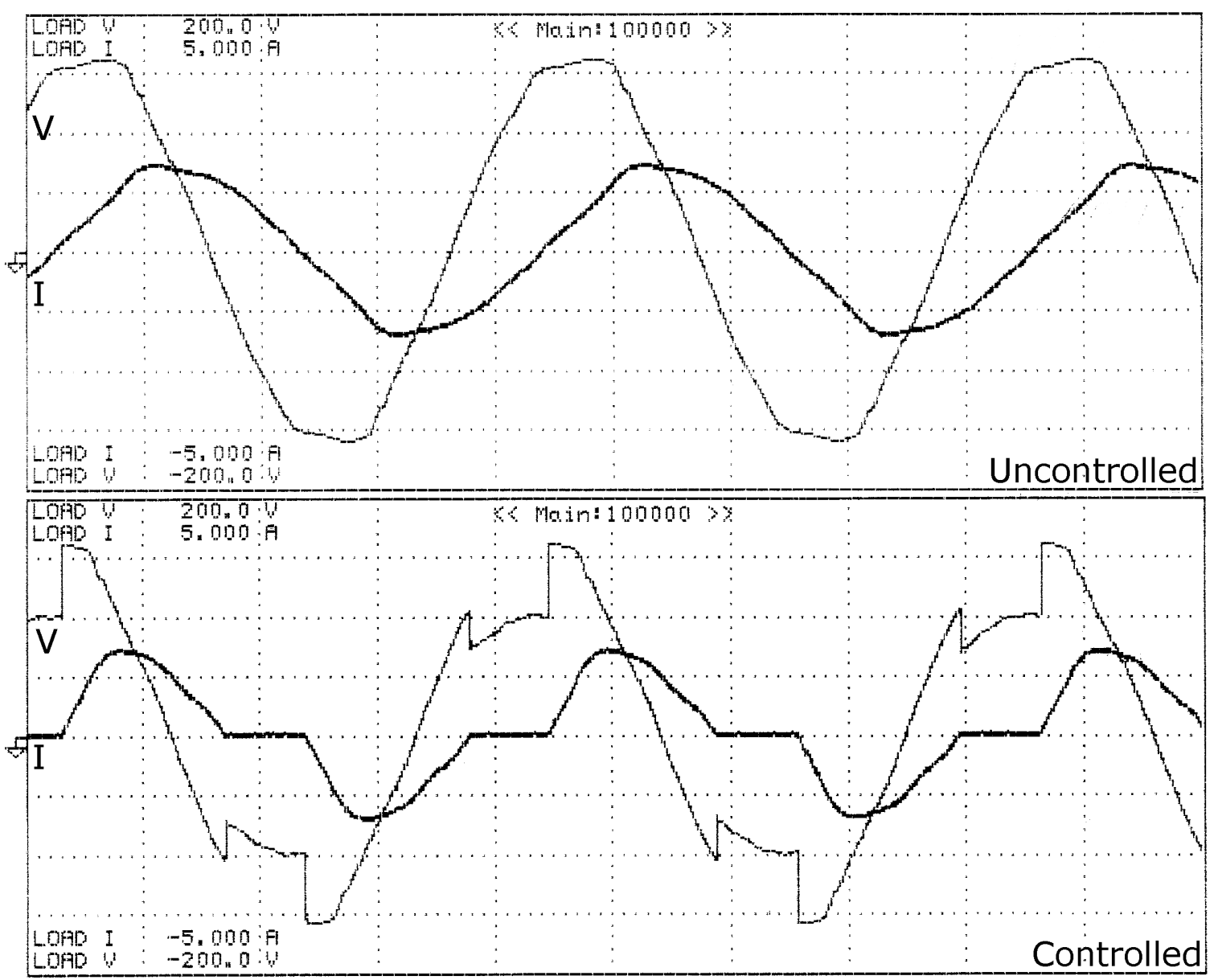

Figure 3.11: Experimental Results, 7 in.-oz. Load 


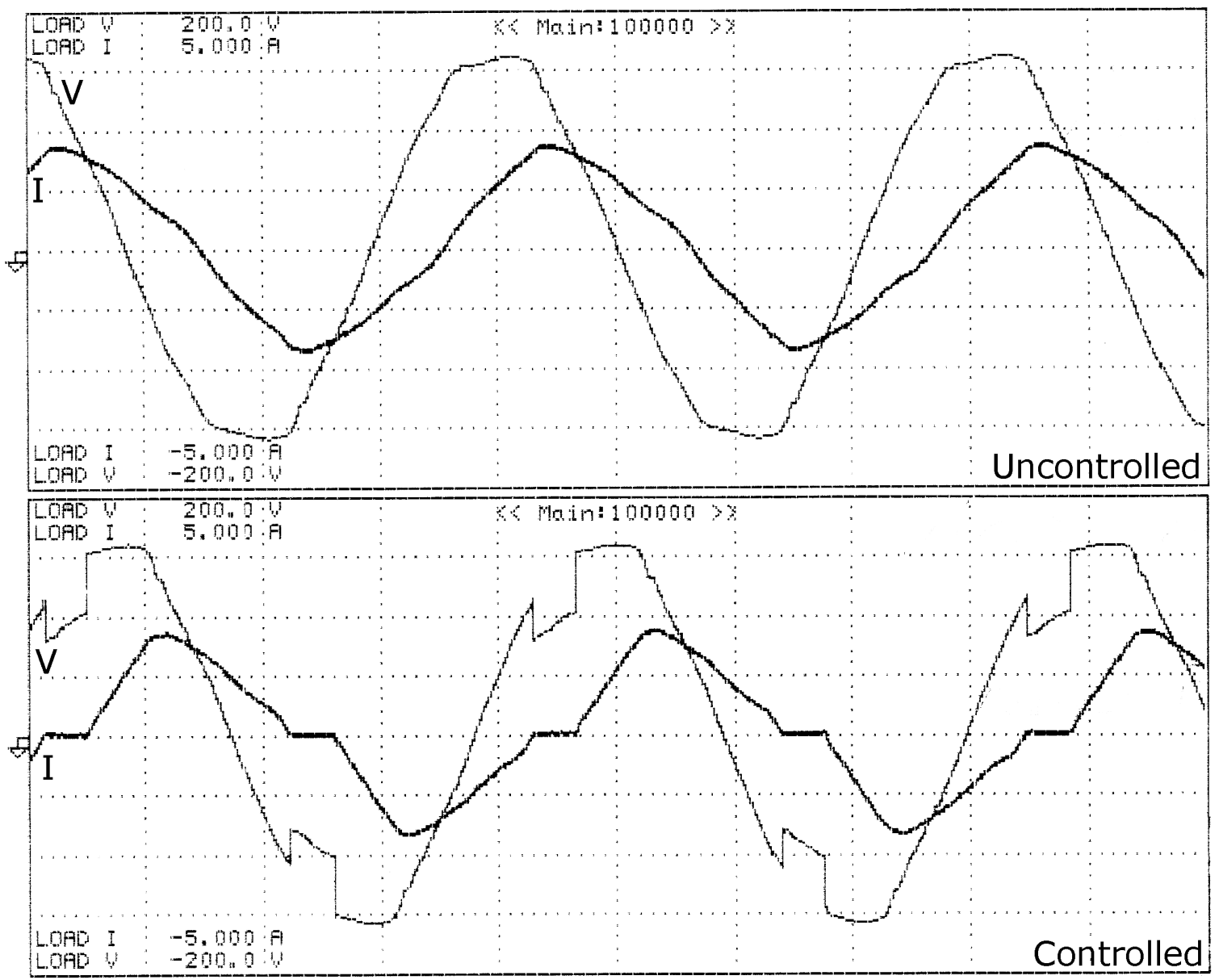

Figure 3.12: Experimental Results, 10 in.-oz. Load 


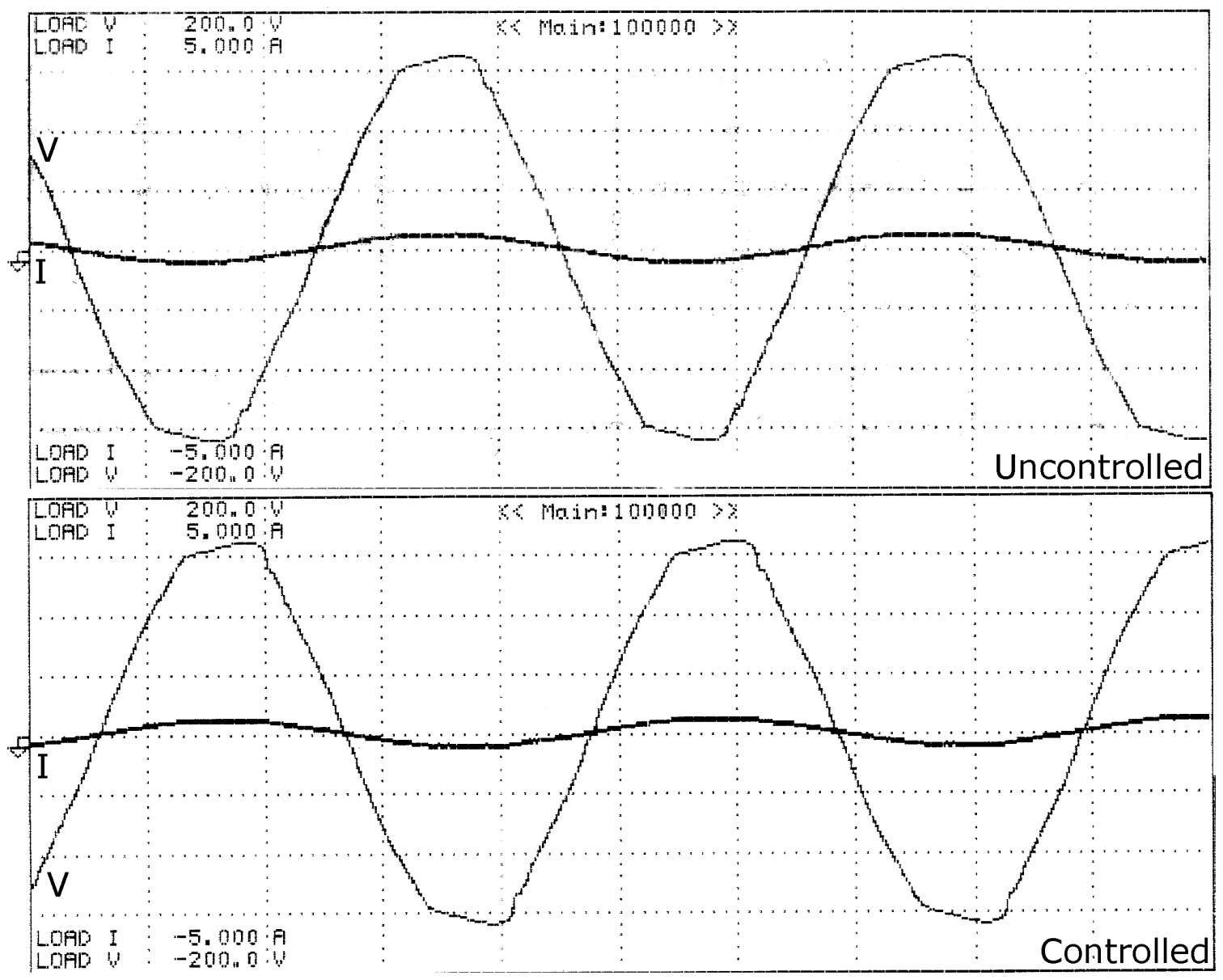

Figure 3.13: Experimental Results, 25W Light Bulb 
the test load setup consisted of tightening a belt around the motor shaft, and this belt was certainly affected by heat and friction. Despite the anomolies, the measurements allow for a good generalization of the motor controller operation and prove that it does in fact function as designed.

The graphs presented effectively illustrate the motor controller's ability to adjust the duty for different load levels. The amount of time the current is at zero between pulses indicates the firing delay on the triac gate, which is inversely proportional to the load level. Comparing Figure 3.10 to Figure 3.12 , it is obvious that the latter shows a much larger current draw, implying a more taxing load condition.

Another important behavior seen in Table 3.2 is the relatively unchanging RPM of the motor, preserving the no-slip property of synchronous motors. It would seem logical that delivering less power to the machine would cause the rotor to slow, but the largest drop in speed was only 18 RPM, a difference of $0.5 \%$. This is especially relevant to applications such as fans that would be less effective at low rotor speeds.

Although reducing the power consumption of an induction motor without significantly affecting its rotation speed is a very beneficial accomplishment, it does not come without a price. The harmonic distortion of the AC line current is greatly increased by the controller, a fact easily observed by comparing the waveforms in Figures 3.10-3.12. Measurements show an average THD increase of $426.6 \%$, and the effect appears to be inversely proportional to the load level.

One result not reflected in the measurements table and voltage/current graphs is the success of the soft start mechanism. During experimentation, it was observed that the motor would power up gradually, run at full for a few seconds, then drop to the appropriate duty. Indicators of the power being used by the motor were audible and verified by watching changes on the power analyzer display.

\subsection{Simulink Model}

In preparation for a full system simulation, a Simulink block diagram of the motor controller was created. The empirical results presented above were used to ensure correctness of the model, as it was impossible to fully recreate the system due to limitations inherent in the simulation environment. However, because the motor control subsystem was tested in hardware and shown to function properly, the model could deviate from the physical implementation. The complete block diagram is shown in Figure 3.14 . 


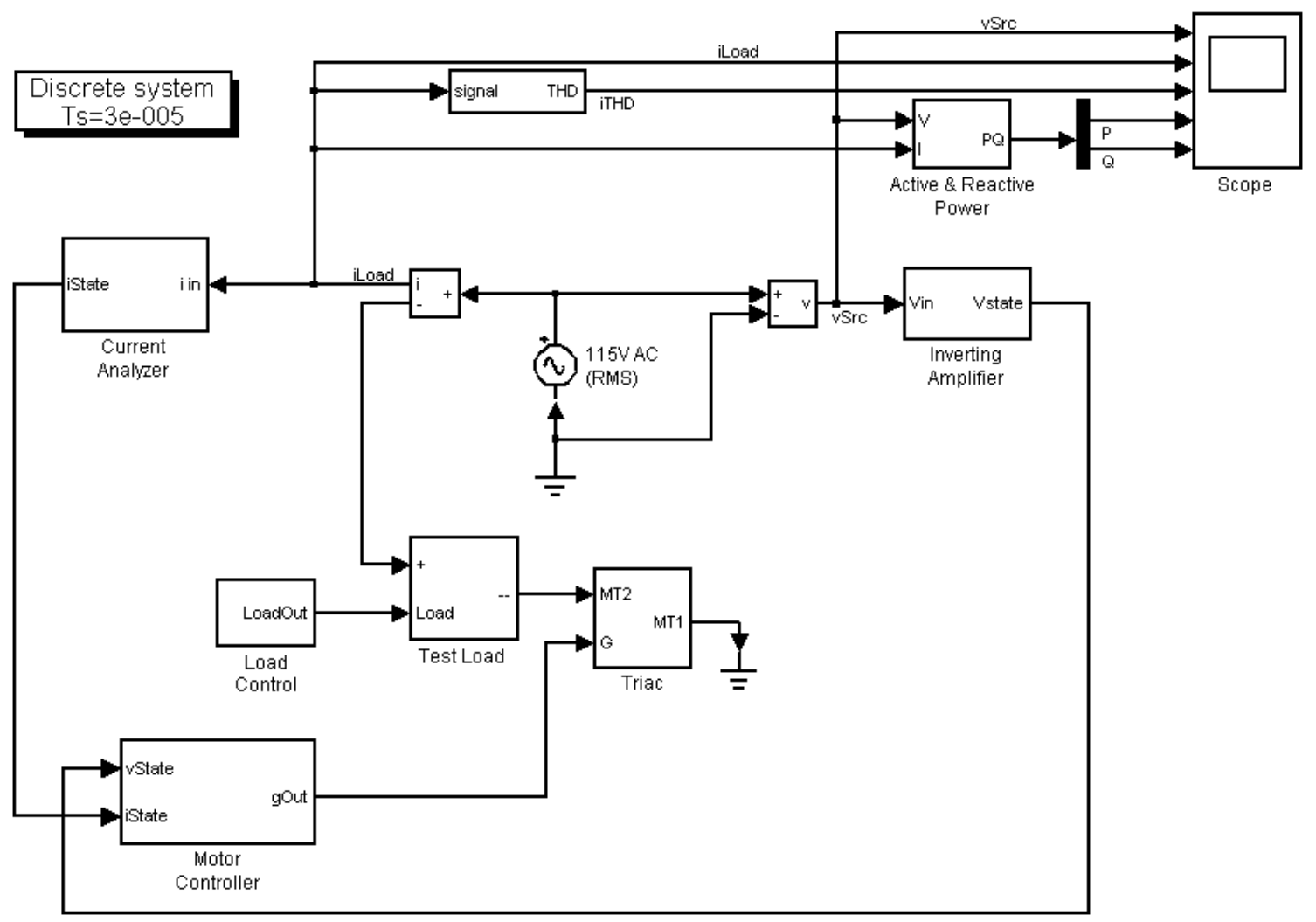

Figure 3.14: Motor Controller Simulink Model

\subsubsection{Triac Model}

One of the aforementioned limitations is the lack of a triac block in the Simulink library. While the wave-chopping functionality could be realized using a simple switch, the logic of the controller would have required alteration. To maintain an open or closed state on a switch block, the control signal must stay constant; this is inconsistent with the pulsing behavior demonstrated in the hardware implementation.

The Power System Blockset for Simulink [26] contains a block for a thyristor, which is a power electronics circuit element that behaves like a gated diode. Because a thyristor only allows current to flow in one direction, it could not be used in place of a triac; however, a triac can be modeled as two opposite-facing thyristors with a common gate [27]. As shown in Figure 3.15, the anode of Thyristor1 and the cathode of Thyristor 2 are tied together to form the MT1 terminal of the triac block. Similarly, the cathode of Thyristor 1 and the anode of Thyristor2 combine to become the MT2 terminal. Both thyristors receive the same gate signal, which may be a pulse 


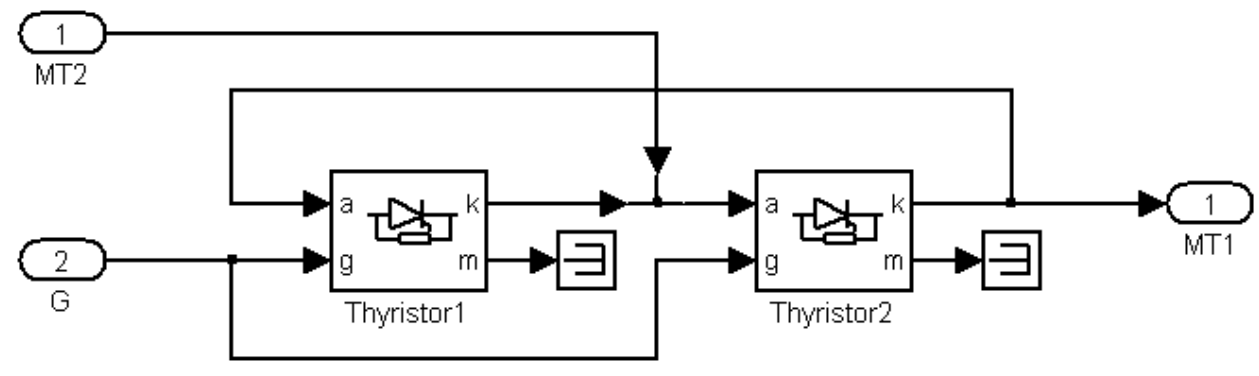

Figure 3.15: Triac Modeled by Thyristors

as was used in the empirical tests.

An additional feature of the thyristor Simulink block is its snubber circuit. The block properties allow input of a resistance and a capacitance to be placed in parallel with the thyristor element. Although a snubber circuit was not used in the hardware implementation, it was discovered that removing the snubbing elements from the Simulink model resulted in much slower simulations. To remedy this, the snubber resistance was set to $10 \mathrm{M} \Omega$ in order to prevent large backward currents. The capacitance was left at the default $4.7 \mu \mathrm{F}$.

While this two-thyristor triac model does function like a real triac, there remains a problem: the gate accepts a logical signal rather than an electrical one. Because the triac gate voltage was used to detect current zero-crossings, an electrical signal would have been more suitable. However, because the zero-crossing detection functionality was successfully demonstrated in hardware, another means for determining when the current waveform crosses zero could be implemented in the simulation.

\subsubsection{Voltage and Current State Detection}

Crucial to the operation of the motor controller is the ability to detect zero-crossings in the voltage and current waveforms. This was accomplished in hardware by using an inverting amplifier to analyze the voltage state and the gate voltage of the triac to determine the current state. The functionality of these two subcircuits was realized in two subsystem blocks created in the Simulink model.

The inverting amplifier block, shown in Figure 3.16, consists of one input, one output, and one simple logic test. If the input voltage (measured and converted to a numeric signal external to this subsystem) is below zero, the output is set to one. During the half-wave when the input voltage is greater than zero, a value of zero is output. This method effectively achieves the same 


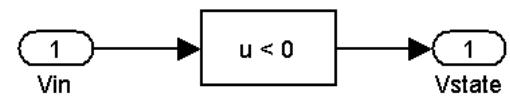

Figure 3.16: Inverting Amplifier Block

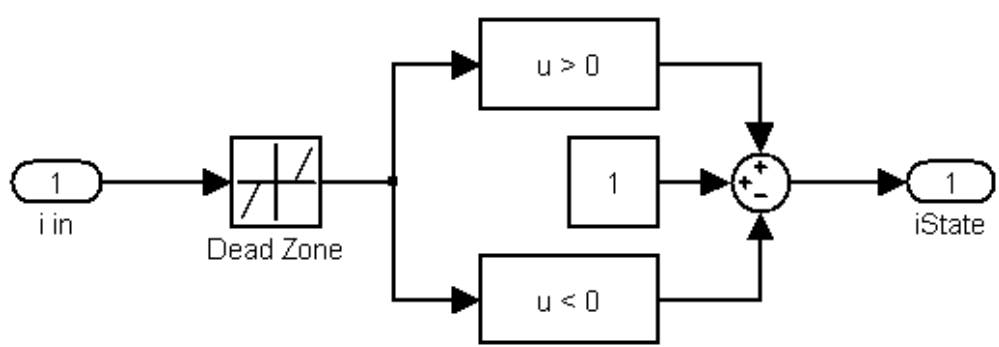

Figure 3.17: Current Analyzer Block

logic levels as the transistor subcircuit in the hardware implementation in a very efficient manner.

Figure 3.17 illustrates the internals of the current analyzer subsystem block. Here, it was desirable to assign a state number corresponding to the instantaneous value of the current waveform. In the physical system, this was done in software running on the PIC microcontroller, but to simulate that would require the additional step of converting the current wave to a triac gate voltage. For simplicity and a more efficient simulation, this step was removed and the controller was passed a state value directly.

As with the inverting amplifier input, the current is measured externally to the analyzer block and converted to a numeric signal. This input is first passed through a dead zone block in order to eliminate noise when the value is near zero. Limits of $-1 * 10^{6}$ and $1 * 10^{6}$ are imposed, and any input value within that range is equated to zero. The output of the dead zone is passed to two logical test blocks to determine if the signal is greater than or less than zero; if the signal is equal to zero, both test blocks also return zero. The summation of the logical tests and a constant one results in an output signal with possible values 0,1 , and 2 that correspond to a current state less than zero, equal to zero, or greater than zero, respectively.

\subsubsection{Controller Model}

The motor controller block is by far the most complicated subsystem in this simulation. Shown in Figure 3.18, the block accepts the voltage and current states as inputs and produces the triac control pulse as an output. 


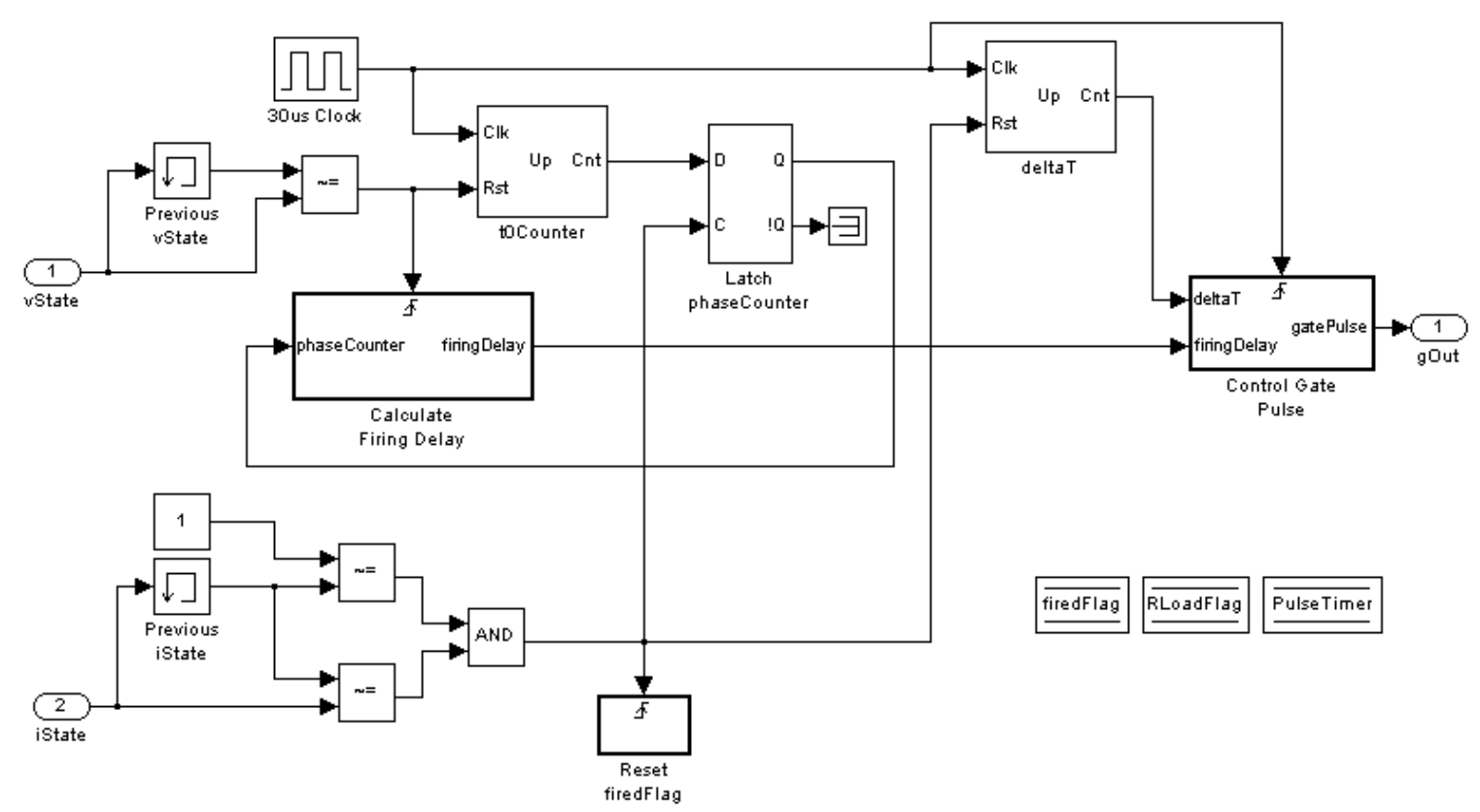

Figure 3.18: Motor Controller Block

Zero-crossings in the voltage waveform are detected by comparing the instantaneous vState input to its last measured value. The block labeled "Previous vState" is a memory unit that outputs the input signal it received one time step previously. Inequality of the current and previous values of the voltage state represent a zero-crossing, which triggers the block labeled "Calculate Firing Delay" and resets a counter.

The "t0 Counter" block represents the variable incremented in each call to the Timer0 interrupt service routine described in Section 3.3.3. The other input to this block is a $30 \mu$ s clock signal; the counter increments its output value on each rising edge of the clock. When a current zero-crossing is detected, the counter output is latched and passed to the firing delay calculation subsystem.

Current zero-crossings are detected using a procedure similar to the one used to detect voltage zero-crossings; however, a change in the iState input over one time step is only half of the condition. As described above, the iState signal is equal to one when the current waveform is equal to zero, and a change from this state to another is caused by a control pulse. The zerocrossing of interest occurs when transitioning to the iState $=1$ condition from another state, so a second inequality comparator is used to isolate these events.

Besides latching the phase counter value, a pulse in the current zero-crossing signal resets the 


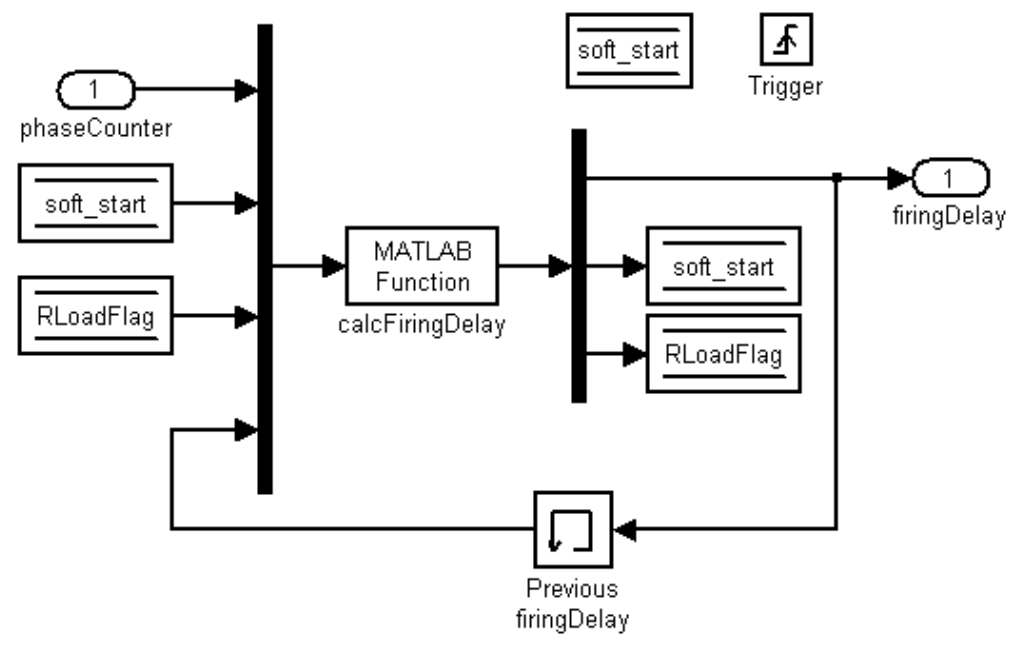

Figure 3.19: Calculation of Firing Delay

firedFlag variable and the deltaT counter. A triggered subsystem block is used to reset the flag; this block contains a data store write to set the flag value to zero. The deltaT counter is controlled by a $30 \mu$ s clock and measures the time passed since a current zero-crossing for comparison with the firing delay value.

Figure 3.19 shows the internals of the "Calculate Firing Delay" triggered subsystem block. The phaseCounter input signal, two data store values, and the previous value of the firing delay are multiplexed and passed to the MATLAB function calcFiringDelay. The complete listing of the source file calcFiringDelay.m can be found in Section A.2.1. A demultiplexer separates the output vector of the function to produce the firingDelay signal and write updated values to the soft_start and RLoadFlag data stores.

The calcFiringDelay function first determines if the system is in soft start mode, and if so the value of firingDelay is checked. If the firing delay is zero (100\% duty), then the value in the soft_start data store is decreased by one; otherwise, the firing delay is decremented. When not in soft start mode, the phase angle is calculated by multiplying phaseCounter by 180 and dividing the result by 277 (the number of $30 \mu$ s intervals in a $60 \mathrm{~Hz}$ half-wave). If the load is not determined to be resistive, then the duty is adjusted as described in Section 3.3 .2 .

Similar to the "Calculate Firing Delay" block, the "Control Gate Pulse" subsystem is shown in Figure 3.20. Triggered every $30 \mu$ s, this block combines the deltaT and firingDelay input signals with three data stores and passes the resulting vector to the gatePulseCtrl MATLAB function, which produces the gatePulse signal to be passed to the triac and updates the firedFlag and 


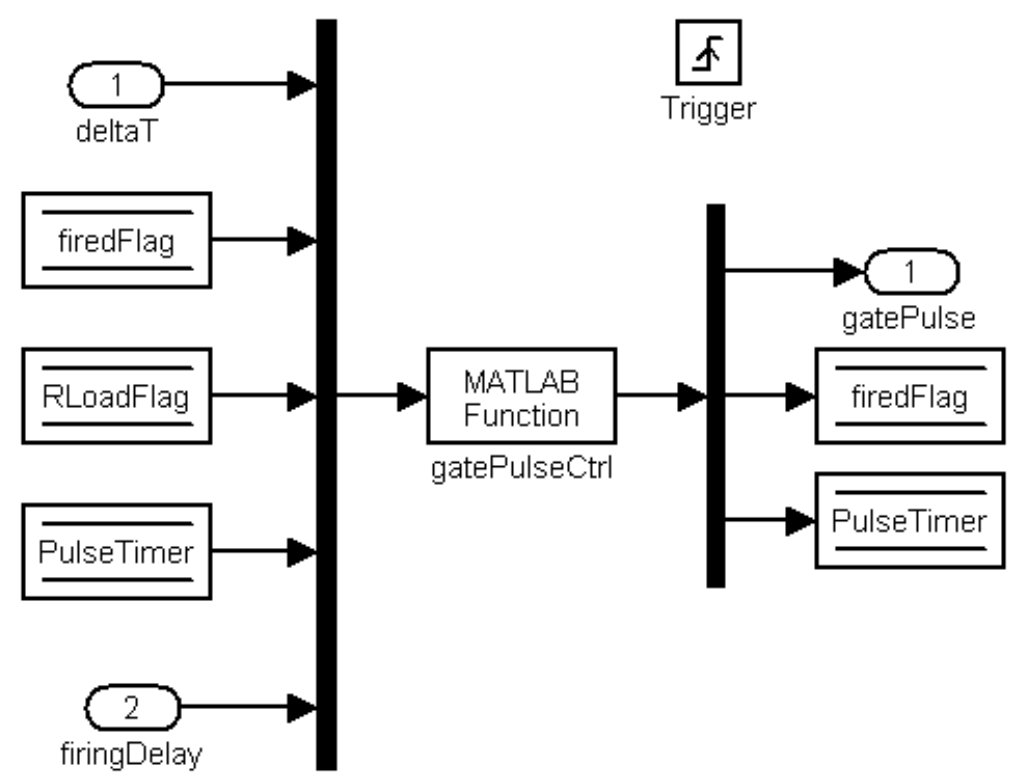

Figure 3.20: Gate Pulse Control Block

PulseTimer data stores. For a complete listing of gatePulseCtrl.m, see Section A.2.2.

The MATLAB code is an adaptation of a portion of the PIC C code used in the Timer0 interrupt service routine. If RLoadFlag is equal to one, the load is resistive and the triac remains in the on state continuously. Otherwise, the action depends on time elapsed and whether or not the triac has been fired. If the value of deltaT is greater than or equal to the firing delay and the triac has not yet been fired, the gate pulse is set to one, the firedFlag variable is set, and the PulseTimer is set to the number of $30 \mu$ s intervals to keep the gate pulse on. Once this has occurred, subsequent calls to the function will decrement PulseTimer by one until it is equal to zero, at which point the triac gate pulse is turned off.

\subsubsection{Test Load}

While the Power System Blockset does provide many useful simulation blocks, its motor models are limited to three-phase and DC implementations. A subsystem based on the singlephase induction motor simulation model in [28] was tested, but the dynamic response of this model was inadequate. Without an induction motor block, it was necessary to produce an alternative test load.

Using emprical data recorded by the Yokogawa power analyzer, the impedance of the mo- 


\begin{tabular}{c|c|c|c|c|c|c} 
Load (in-oz) & $\mathrm{V}_{R M S}(\mathrm{~V})$ & $\mathrm{I}_{R M S}(\mathrm{~A})$ & $\Phi\left(^{\circ}\right)$ & $\mathrm{Z}_{\text {load }}$ & $\mathrm{R}_{\text {load }}(\Omega)$ & $\mathrm{L}_{\text {load }}(\mathrm{mH})$ \\
\hline 0 & 115.783 & 1.2113 & -75.89 & $23.0267+\mathrm{j} 91.6053$ & 23.0267 & 242.991 \\
6 & 116.439 & 1.2258 & -70.09 & $32.3483+\mathrm{j} 89.3125$ & 32.3483 & 236.909 \\
7 & 116.119 & 1.2323 & -66.34 & $37.8151+\mathrm{j} 86.3088$ & 37.8151 & 228.941 \\
8 & 115.995 & 1.3400 & -53.83 & $51.0883+\mathrm{j} 69.8800$ & 51.0883 & 185.362 \\
9 & 116.031 & 1.3679 & -52.34 & $51.8254+\mathrm{j} 67.1511$ & 51.8254 & 178.124 \\
10 & 115.896 & 1.4191 & -49.01 & $53.5687+\mathrm{j} 61.6455$ & 53.5687 & 163.52
\end{tabular}

Table 3.3: Impedance of Motor Load

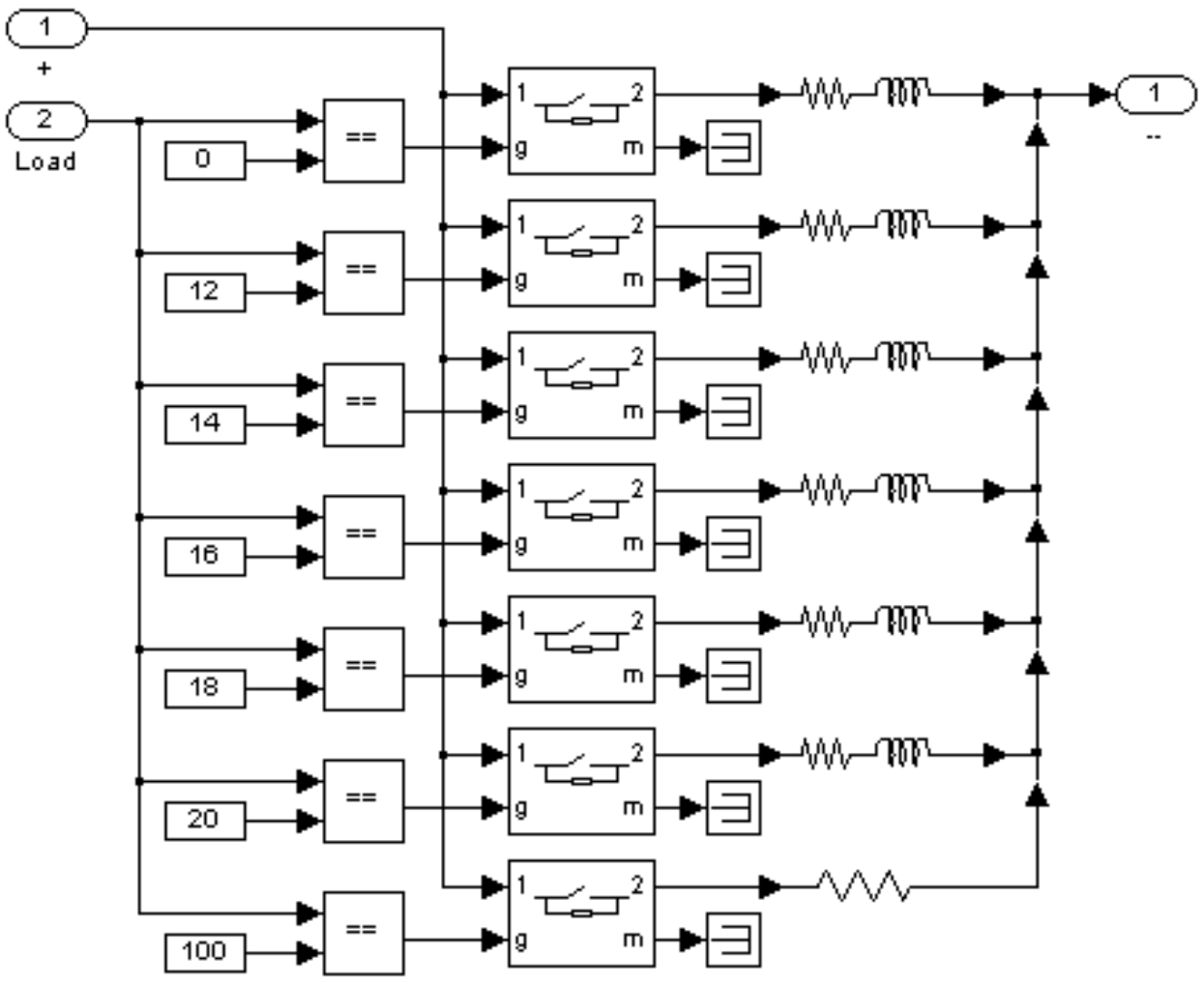

Figure 3.21: Test Load Block

tor was determined at several load levels. The RMS voltage and current levels used for the calculations were measured while the motor was running without being connected to the controller. Table 3.3 shows the impedence, resistance, and inductance for the motor at each load measurement.

The implementation of the test load is shown in Figure 3.21. Positive and negative terminals are provided for connecting the block to the main circuit, and a load selection input activates the 
appropriate RL branch. By using comparator blocks and switches, the load selection signal can be changed externally, allowing simulations that test the adjustment features of the controller.

When the load level input signal is equal to 100 , a $100 \Omega$ resistor is connected to simulate a purely resistive load. The value of the resistor value was chosen arbitrarily and does not match the resistance of the $25 \mathrm{~W}$ light bulb used for hardware testing. It should also be noted that no simulations were run that included both a "motor" and a resistor in the load.

\subsubsection{Test Results}

Table 3.4 and Figures 3.22 - 3.25 show the measurements from the motor controller simulation and selected waveforms. The load levels used for the first three graphs are the same values used in the hardware test. For each load, the simulation was run for five seconds, and the last $50 \mathrm{~ms}$ were used to calculate averages and plot graphs. Voltage and current values are RMS, while THD, P, and $\mathrm{Q}$ are mean values.

The simulation results appear much better than their real-world counterparts, which is to be expected since the simulation is run in an ideal environment. Also, without the nonlinearities of a true motor model, these numbers and graphs will not be completely accurate. However, the results do show that the motor controller logic functions as designed.

Active and reactive power calculations in the Simulink model may differ from those used by the Yokogawa power analyzer. The Power System Blockset documentation states that harmonics are accounted for and that the values of $\mathrm{P}$ and $\mathrm{Q}$ are calculated at the user-input fundamental frequency. A moving window is used to average the instantaneous $\mathrm{V}^{*} \mathrm{I}$ values over one period, though this is not a root-mean-square average [26]. While the empirical and simulated calculations may vary, the $\mathrm{P}$ and $\mathrm{Q}$ determined by Simulink are acceptable since they are affected by harmonics and nonlinear waveforms.

Trends in the simulated data confirm those seen in the empirical results. Percentage reductions in current draw and real power decrease with increasing load, and harmonic distortion due to the controller is worse under lighter loads. The controller successfully detected a resistive load and allowed it to run at full duty.

With a fully-functional motor controller simulation completed, the next step was to address the issue of harmonic distortion. The next chapter details the line conditioner subsystem that was added to the motor controller and the results of the complete system simulation. 


\begin{tabular}{|c|c|c|c|c|c|}
\hline & $\mathrm{I}_{R M S}(\mathrm{~A})$ & $\mathrm{I}_{T H D}(\%)$ & $\mathrm{P}(\mathrm{W})$ & $\mathrm{Q}$ (VAR) \\
\hline \multirow[t]{3}{*}{ No load } & Uncontrolled & 1.2176 & 0.46 & 34.1416 & 135.8551 \\
\hline & Controlled & 0.2297 & 65.83 & 1.3255 & 21.8731 \\
\hline & Difference & 0.9879 & -65.37 & 32.8161 & 113.982 \\
\hline \multirow[t]{3}{*}{6 in.-oz. } & Uncontrolled & 1.2108 & 0.46 & 47.4202 & 130.9964 \\
\hline & Controlled & 0.2541 & 63.03 & 2.1978 & 24.4541 \\
\hline & Difference & 0.9567 & -62.57 & 45.2224 & 106.5423 \\
\hline \multirow[t]{3}{*}{7 in.-oz. } & Uncontrolled & 1.2205 & 0.46 & 56.3312 & 128.6632 \\
\hline & Controlled & 0.2809 & 60.64 & 3.0947 & 27.2118 \\
\hline & Difference & 0.9396 & -60.18 & 53.2365 & 101.4514 \\
\hline \multirow[t]{3}{*}{8 in.-oz. } & Uncontrolled & 1.3286 & 0.46 & 90.1746 & 123.5144 \\
\hline & Controlled & 0.4483 & 50.52 & 10.4018 & 44.5161 \\
\hline & Difference & 0.8803 & -50.06 & 79.7728 & 78.9983 \\
\hline \multirow[t]{3}{*}{9 in.-oz. } & Uncontrolled & 1.3558 & 0.46 & 95.2645 & 123.6184 \\
\hline & Controlled & 0.4924 & 48.31 & 12.7133 & 49.0636 \\
\hline & Difference & 0.8634 & -47.85 & 82.5512 & 74.5548 \\
\hline \multirow[t]{3}{*}{10 in.-oz. } & Uncontrolled & 1.4082 & 0.46 & 106.2242 & 122.4464 \\
\hline & Controlled & 0.5966 & 43.48 & 19.4414 & 59.9067 \\
\hline & Difference & 0.8116 & -43.02 & 86.7828 & 62.5397 \\
\hline \multirow[t]{3}{*}{$100 \Omega$ resistor } & Uncontrolled & 1.1499 & 0.46 & 132.2566 & 0.2717 \\
\hline & Controlled & 1.1427 & 0.48 & 131.4269 & 0.0569 \\
\hline & Difference & 0.0072 & -0.02 & 0.8297 & 0.2148 \\
\hline
\end{tabular}

Table 3.4: Motor Controller Simulation Results 

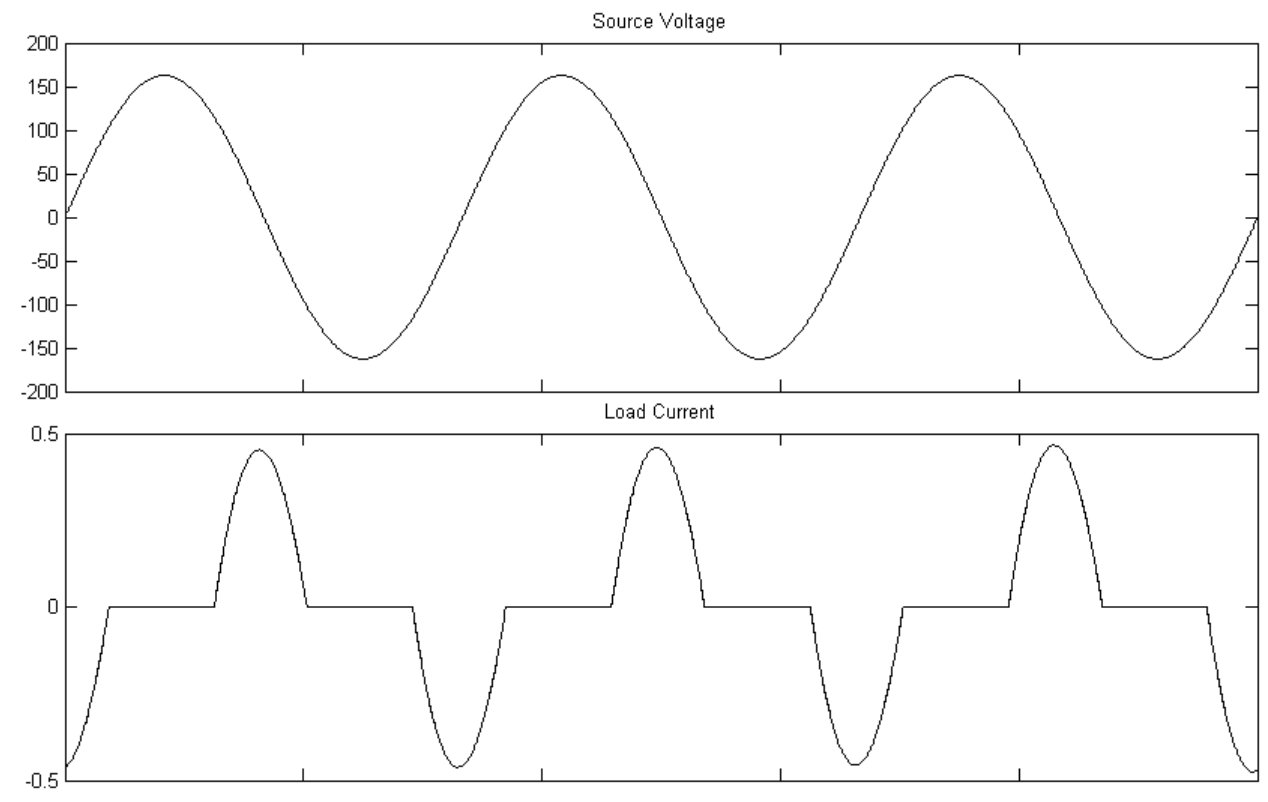

Figure 3.22: Simulated Results, No Load
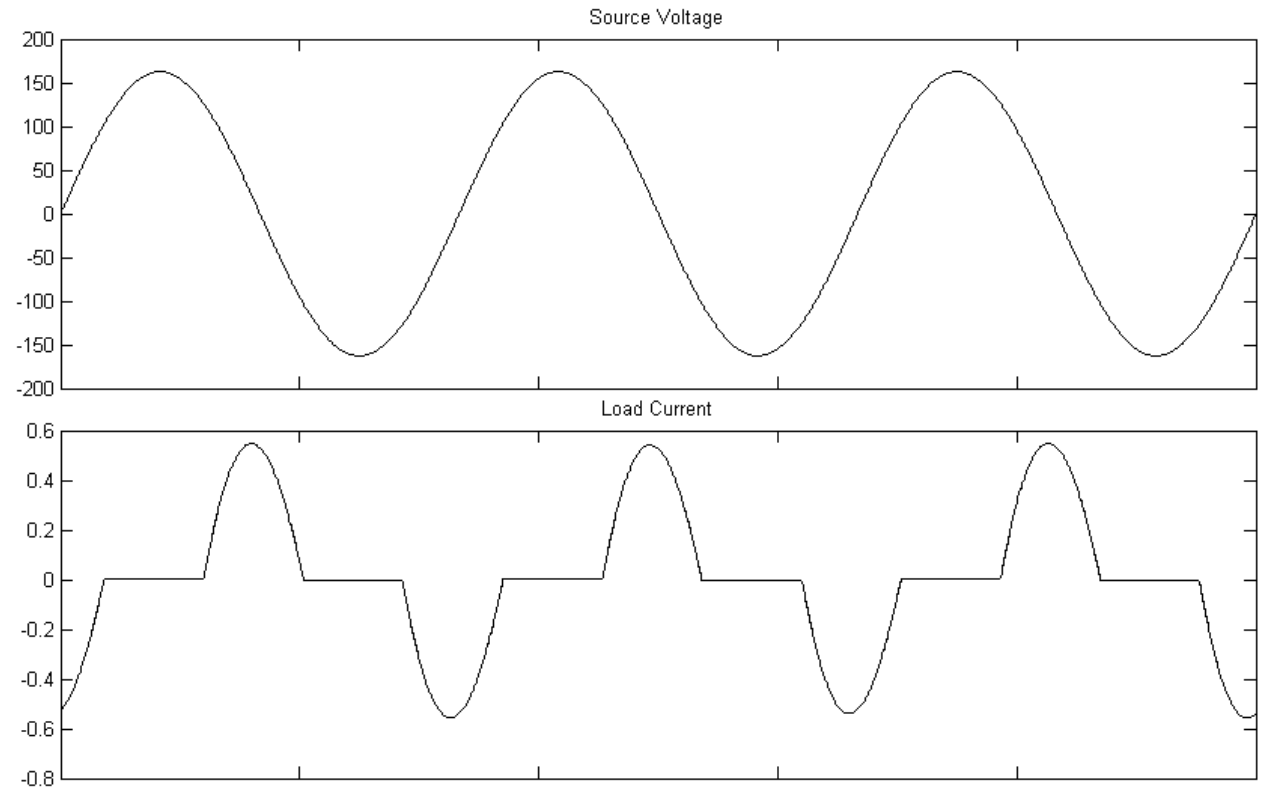

Figure 3.23: Simulated Results, 7 in.-oz. Load 

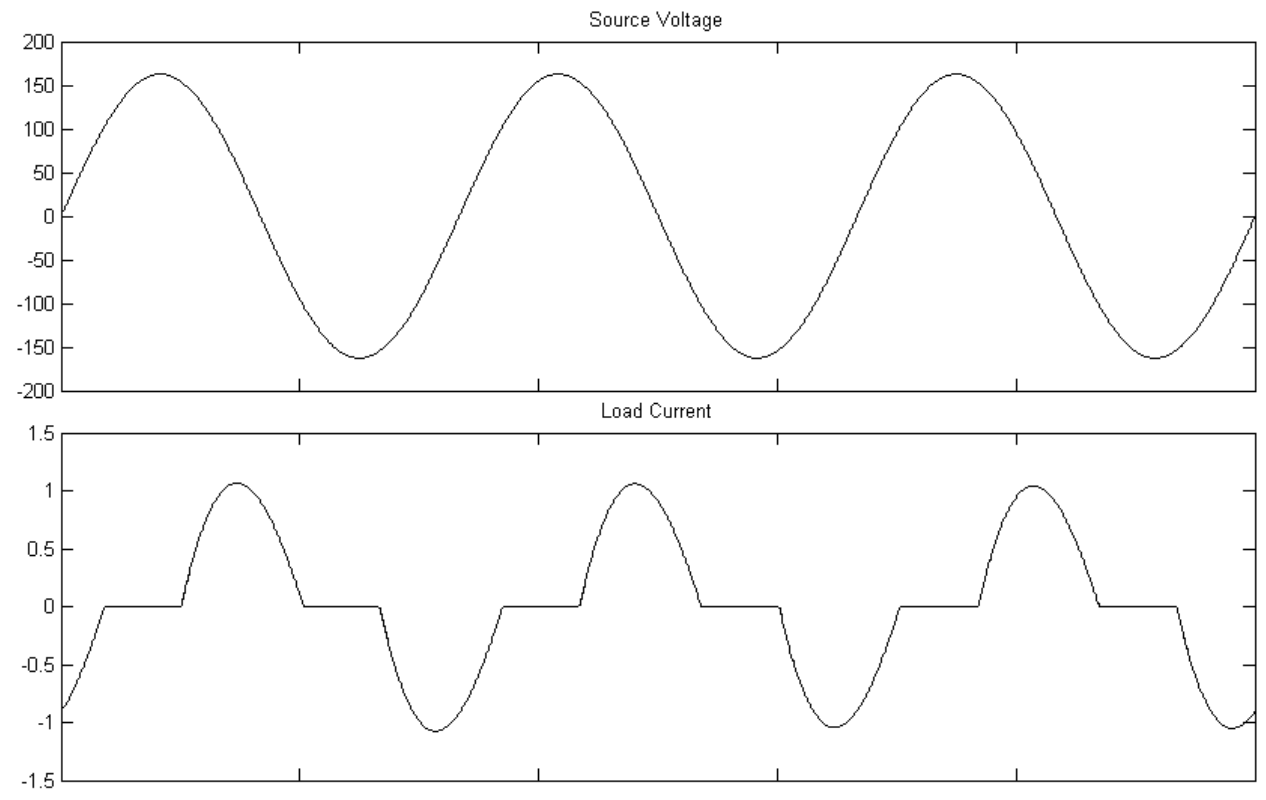

Figure 3.24: Simulated Results, 10 in.-oz. Load
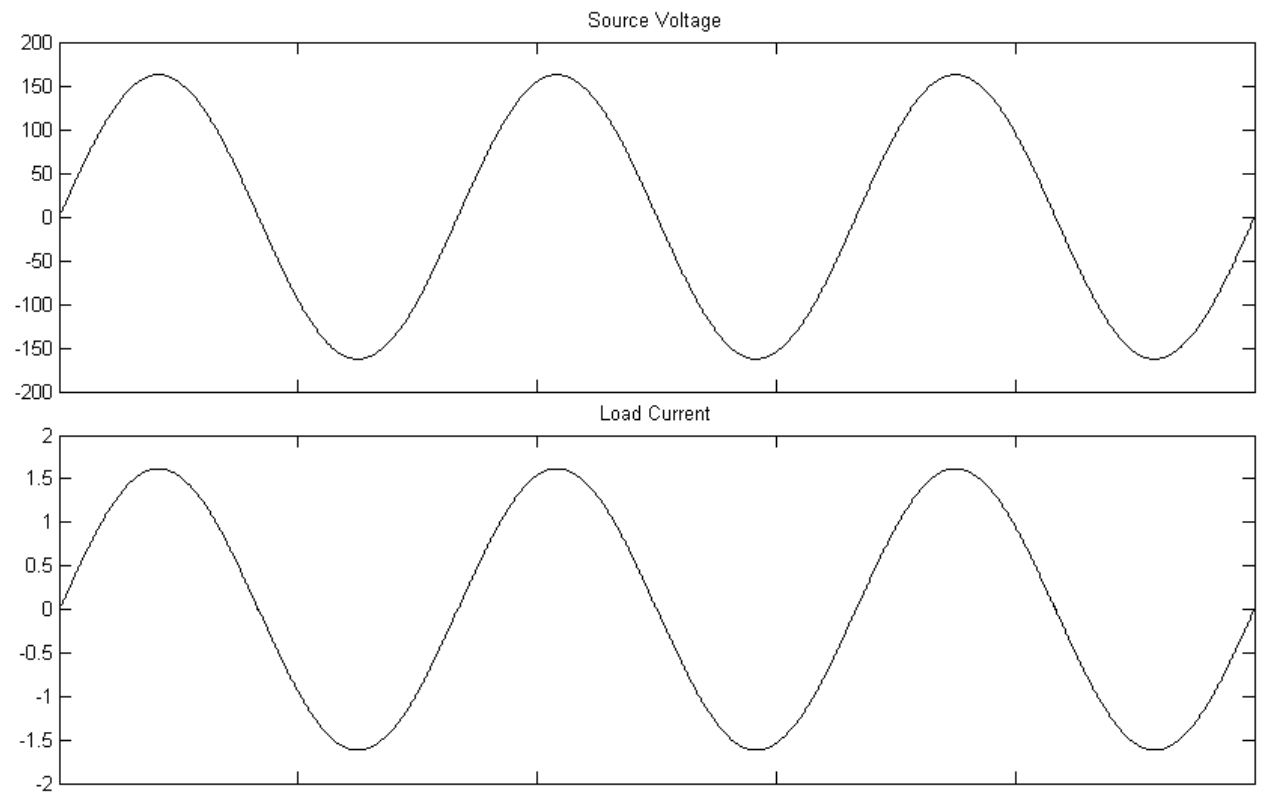

Figure 3.25: Simulated Results, Resistive Load 


\section{Chapter 4}

\section{Line Conditioner}

The results presented in in Sections 3.4 and 3.5 .5 clearly illustrate the need for power quality conditioning. This chapter details the addition of a line conditioner to the motor controller described in the previous chapter. Concepts and an existing design are presented, followed by a discussion of adapting and simulating the subsystem. Finally, a full system simulation model is discussed and results are given.

\subsection{Active Power Quality Conditioner}

Figure 4.1 shows the circuit diagram for the line conditioner presented in [29]. This design uses a DC capacitor to store energy from the AC source and provide compensation to the system when needed. The inductor in series with the source establishes a synchronous link, which is used to control the line conditioner output and isolate the AC line from harmonics [29]. It should be noted that the load is not shown in Figure 4.1; it would be located in parallel with filter capacitor $\mathrm{C}_{f}$, to the left of the inductor $\mathrm{L}$, as shown in Figure 4.2 .

\subsubsection{Description}

The DC voltage on the capacitor is passed through an inverter as a first step toward conversion to an AC signal. Such an inverter is made up of four switching elements, and is typically controlled by pulse-width modulation (PWM). The output of the inverter is fed to a filter, which smoothes out the pulses and creates a sine wave.

In [29], Kumar suggests three methods of controlling the inverter: maintain a constant voltage across the DC capacitor, directly alter the current produced by the inverter, or calculate and 


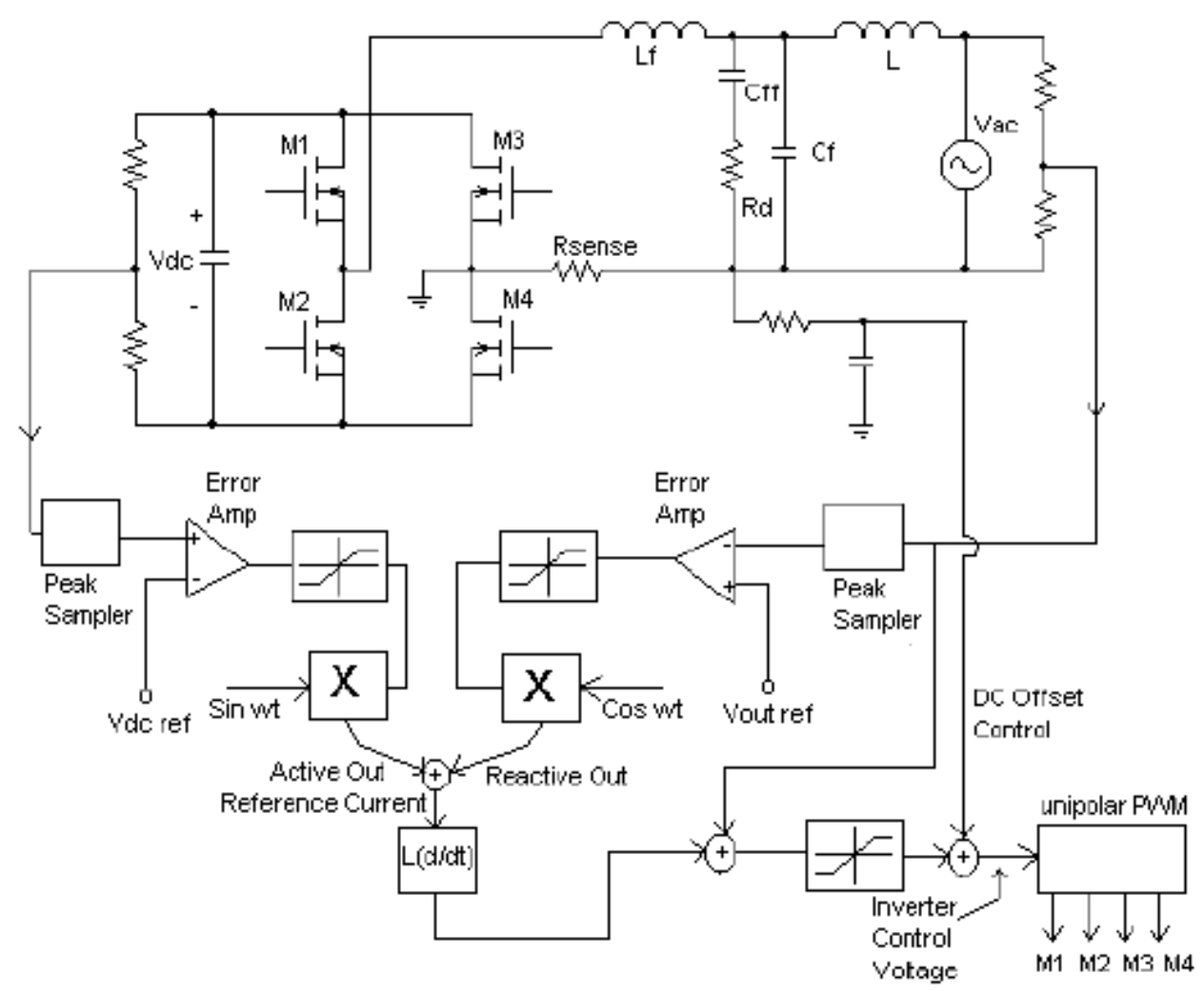

Figure 4.1: Active Power Quality Conditioner Circuit (From [29])

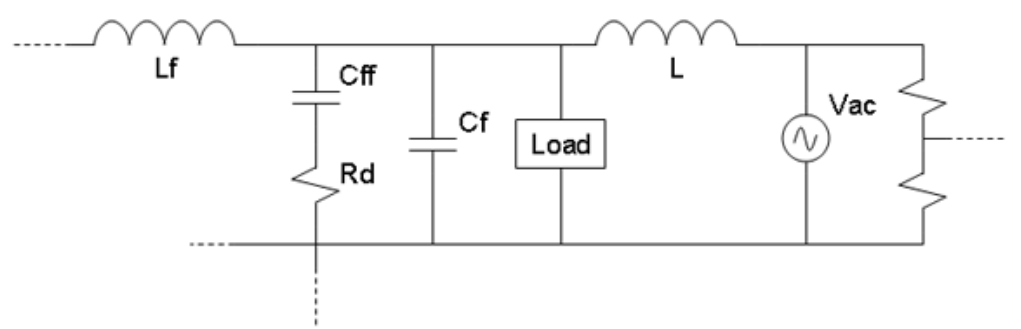

Figure 4.2: Active Power Quality Conditioner Load Detail 


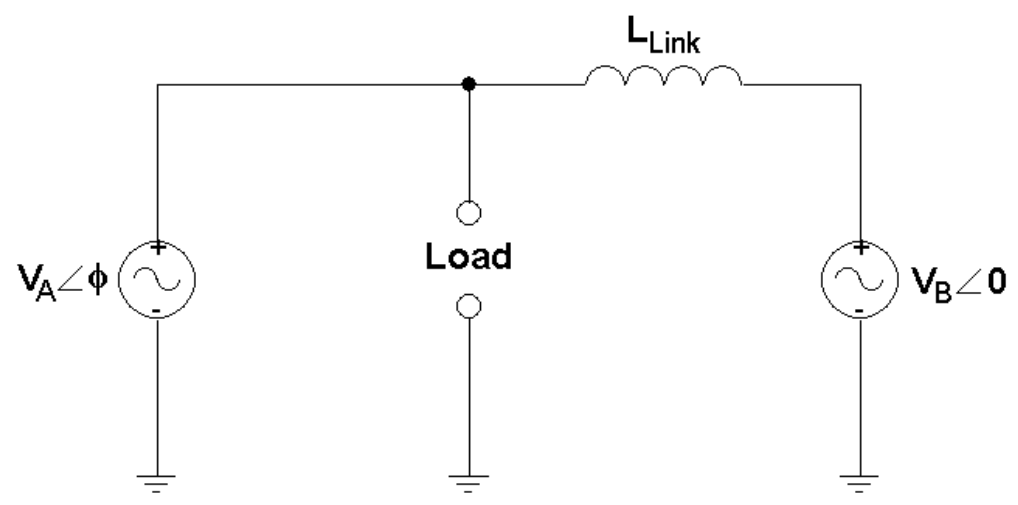

Figure 4.3: Synchronous Link Circuit

produce the required inverter output voltage. The third control scheme is used in Kumar's design, and is achieved by determining the active and reactive currents to be drawn by the inverter, then calculating the voltage drop across the link reactor. Samples of the DC capacitor voltage and inverter output voltage are taken and compared with reference values, then sine and cosine template waveforms are amplitude modulated by the voltage differences. Knowing the current needed and the impedance of the link reactor allows the required inverter output voltage to be calculated as $V_{A C}+L \frac{d i}{d t}[29]$.

A simplification of the synchronous link concept is shown in Figure 4.3, and is comprised of two AC voltage sources that are out of phase by some angle $\phi$. In the active power quality conditioner, $\mathrm{V}_{A}$ represents the $\mathrm{DC}$ capacitor and inverter, whereas $\mathrm{V}_{B}$ is the $\mathrm{AC}$ line voltage. It can be shown that real power flows from leading to lagging source in direct proportion to $\sin (\phi)$, and reactive power flows from high to low voltage, proportional to the voltage difference [29].

\subsection{Simulink Simulation}

Kumar presents block diagrams and results for simulation of the active power quality conditioner using Microsim Design Lab in [29]. As can be seen from Figure 4.1, analog circuit elements are used to control the system. Kumar's line conditioner was replicated in Simulink to ensure proper understanding and operation of the controller, then the control scheme was altered to utilize a microcontroller. The conversion to digital control is covered in Section 4.3 . 


\subsubsection{Simulink Model}

The Simulink implementation of the line conditioner shown in Figure 4.4 uses the same analog techniques as Kumar's model. The model was tested with an RL load switched on and off by a triac, in anticipation of combining the conditioner with the motor controller.

On the right side of the model, a $160 \mathrm{~V}$ (115V RMS) AC voltage source is connected to the link reactor, which has components of $100 \mathrm{mH}$ and $2 \Omega$. The AC source voltage is measured and passed to the controller block as an input. A $330 \mu \mathrm{F}$ capacitor serves as the input voltage for the inverter block, and the voltage across the terminals of the capacitor is also passed to the controller. The neutral node shown was necessary to connect the input port of the capacitor to the input port of the inverter.

The controller block passes a control signal to the inverter, which uses the energy stored in the DC capacitor to create a sinusoidal voltage source. This is then combined with the $160 \mathrm{~V}$ AC signal and passed to the load. A switch is used to keep the load disconnected until 100ms into the simulation, allowing the DC capacitor time to reach its nominal charge of $250 \mathrm{~V}$. The triac block uses the two-thyristor model described in Section 3.5.1 and is controlled by a pulse generator with a period of $1 / 120 \mathrm{~s}$, a phase delay of $6 \mathrm{~ms}$, and a pulse width of $1 \%$.

Figure 4.5 shows the "Controller" subsystem in detail. The DC capacitor voltage is compared to its nominal value of $250 \mathrm{~V}$, and the difference is saturated to be no more than $50 \mathrm{~V}$ in either direction. This signal represents $\mathrm{dV}$ in the capacitor current equation, $I=C * \frac{d V}{d t}$, and a negative value indicates that the capacitor requires more energy from the $\mathrm{AC}$ voltage source. While the sign may seem counterintuitive, the controller calculates the current output of the line conditioner, so a negative value results in a current that flows inward and charges the capacitor.

Next, the voltage difference is multiplied by the capacitor value and divided by the sample time. The result is the amount of current required to pass through the capacitor, which is latched at $240 \mathrm{~Hz}$ using a D-latch block. The next multiplier combines the capacitor current value with a sine wave template, converting the signal from a DC current to an AC current. The sine wave template has unity amplitude and a $60 \mathrm{~Hz}$ frequency; it is designed to be exactly in phase with the AC source voltage signal. The output of the multiplier represents the required active current to be sourced from the line conditioner.

The output current must be converted to a voltage so that the controller can provide proper signals to the DC-to-AC inverter. To do so, the output current value is passed through a numerical derivative block, then multiplied by the value of the link reactor inductance. The small 


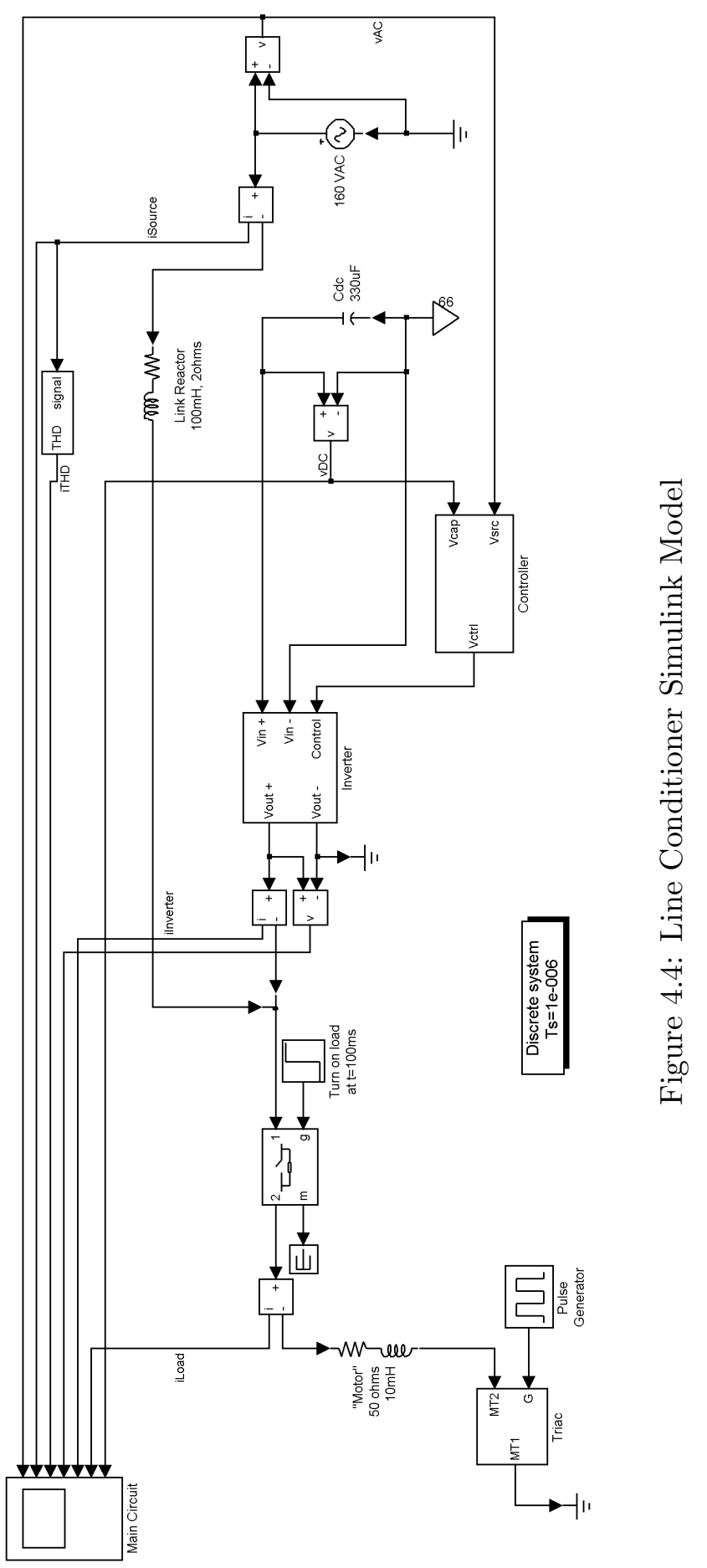




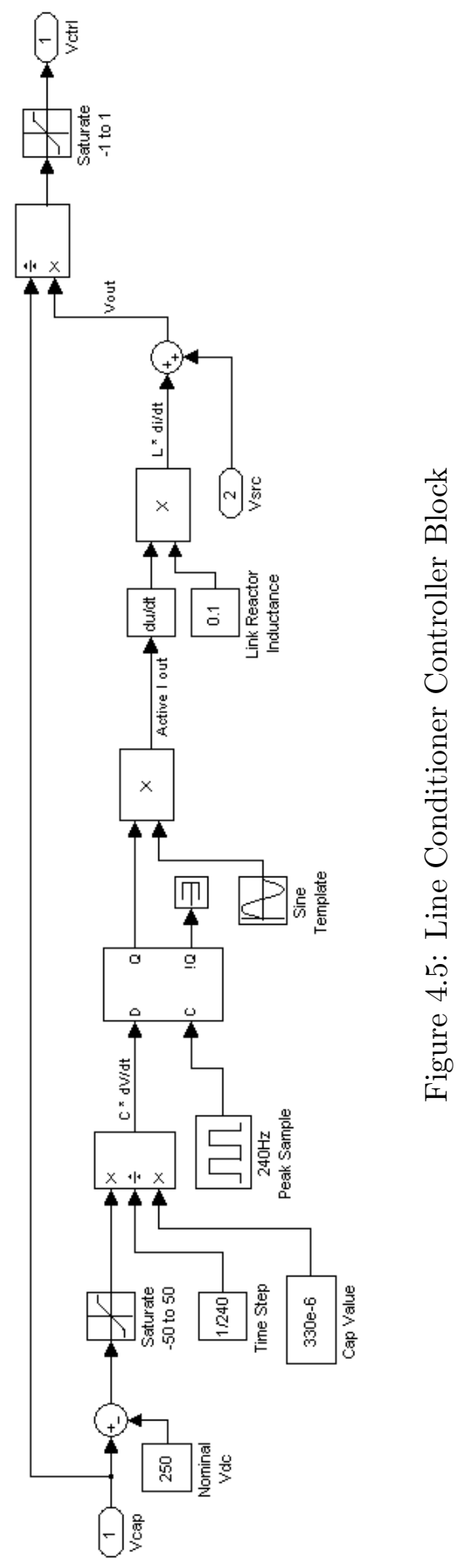




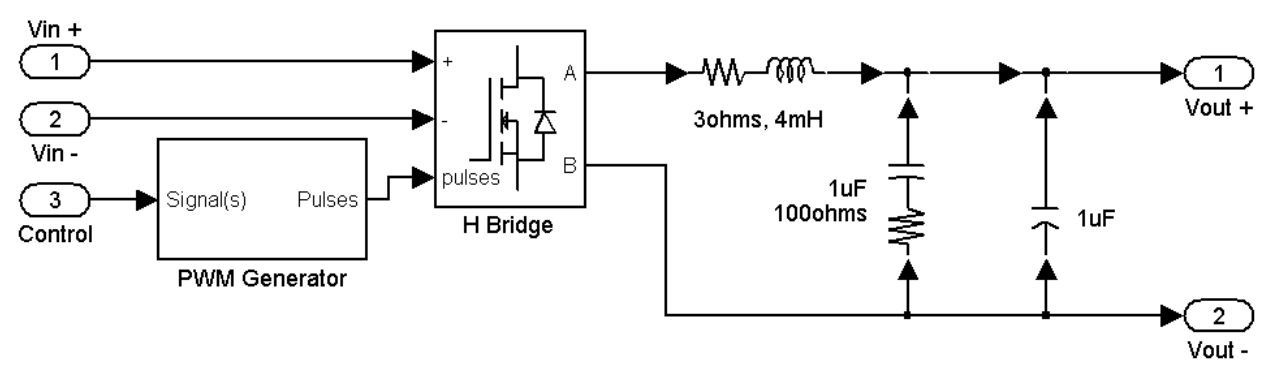

Figure 4.6: Line Conditioner Inverter Block

resistive component of the link reactor is not used in the caluclation because the inductive element dominates the impedance. This multiplier block provides as an output the voltage across the link reactor per the formula $V=L * \frac{d I}{d t}$.

Summing the link reactor voltage and the AC source voltage results in the required output voltage of the line conditioner. The final step to producing the required control signal is to normalize the output voltage by dividing by the voltage across the DC capacitor. A saturation block is used to keep the result of the division between -1 and 1 , and the signal is passed to the output port of the controller block.

The output of the line conditioner controller is fed to the inverter block, shown in detail in Figure 4.6. The control signal is passed to a PWM generator, which compares its internal carrier wave to the input and produces the appropriate pulses to drive a bridge. For this simulation, the carrier wave frequency was set to $5 \mathrm{kHz}$, and the generator was set up to produce four pulses to drive a two-arm bridge.

Pulses from the PWM generator are fed to the H-bridge block. The Power System Blockset provides a generic bridge block that can be set up in several configurations. For this system, the bridge is composed of MOSFETs, and each of the four MOSFET elements has a protective diode and RC snubber connected in parallel. This block effectively converts the DC capacitor voltage to a series of pulses that has a variation in width corresponding to the desired value of a sinusoidal voltage signal.

To convert the pulse output of the H-bridge to an AC voltage, a passive RLC filter is used. The values of the circuit elements were taken directly from [29], as can be seen in Figure 4.1. 


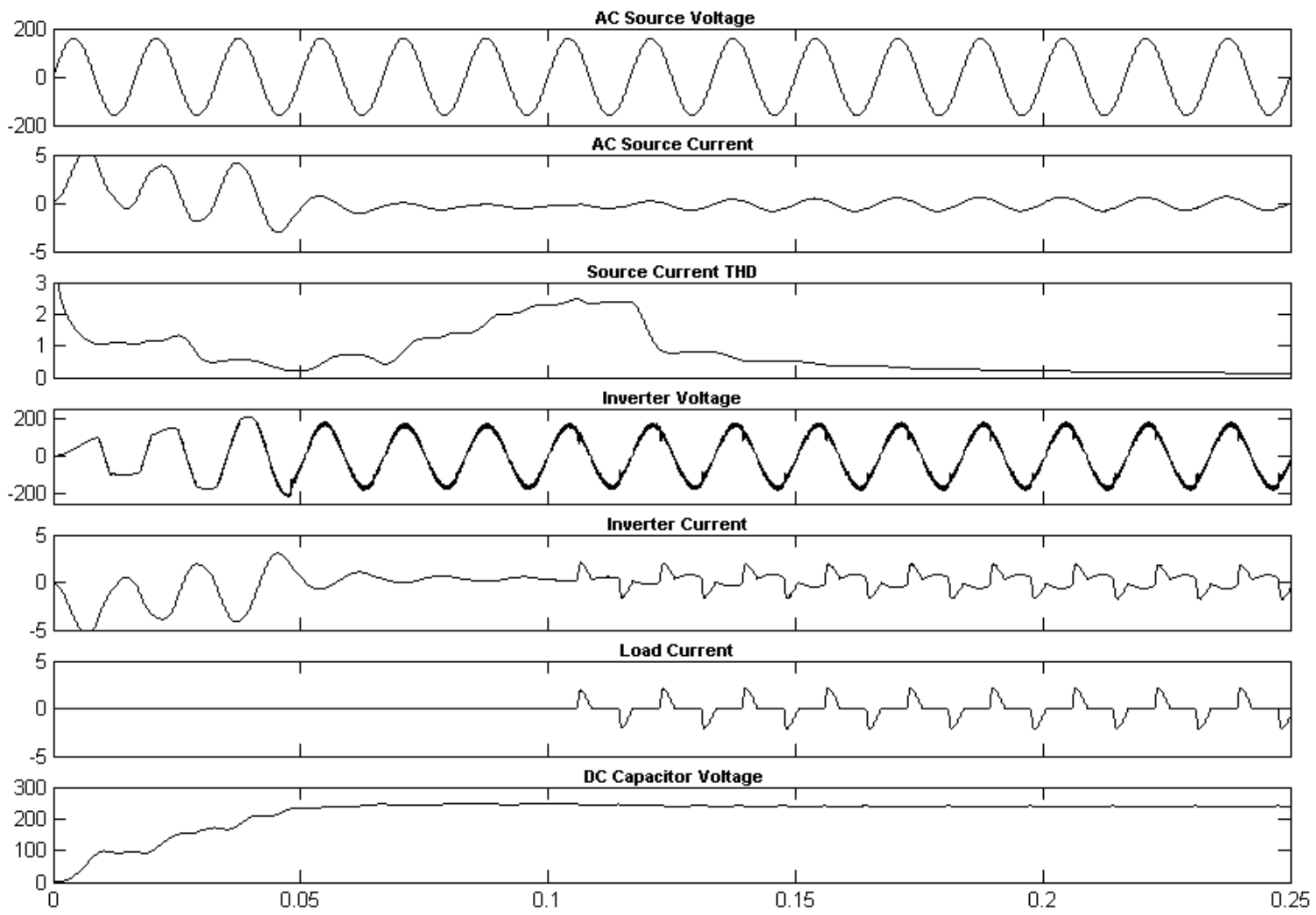

Figure 4.7: Analog Line Conditioner Scope Traces

\subsubsection{Results}

The simulation was run for 0.25 seconds, with the load disconnected for the first $100 \mathrm{~ms}$. This was done to allow the DC capacitor to charge and produce a steady sinusoidal voltage output from the inverter. Resulting waveforms are shown in Figure 4.7, which may be compared to the current and THD waveforms of the unconditioned load presented in Figure 4.8. Note that THD is expressed in Simulink as the RMS value of the harmonic components divided by the RMS value of the fundamental component [26]: multiplying by 100 will give the THD as a percentage.

Near the start of the simulation, the AC source current waveform is offset and somewhat deformed, as is further evidenced by the harmonic distortion curve. Once the load is switched on, however, the THD of the current sharply decreases, then continues to steadily drop as the system stabilizes. The average THD between $0.15 \mathrm{~s}$ and $0.2 \mathrm{~s}$ is $31.11 \%$, and the average improves to $16.89 \%$ between $0.2 \mathrm{~s}$ and $0.25 \mathrm{~s}$.

The load current appears as expected, and the switching nature of the triac is compensated 

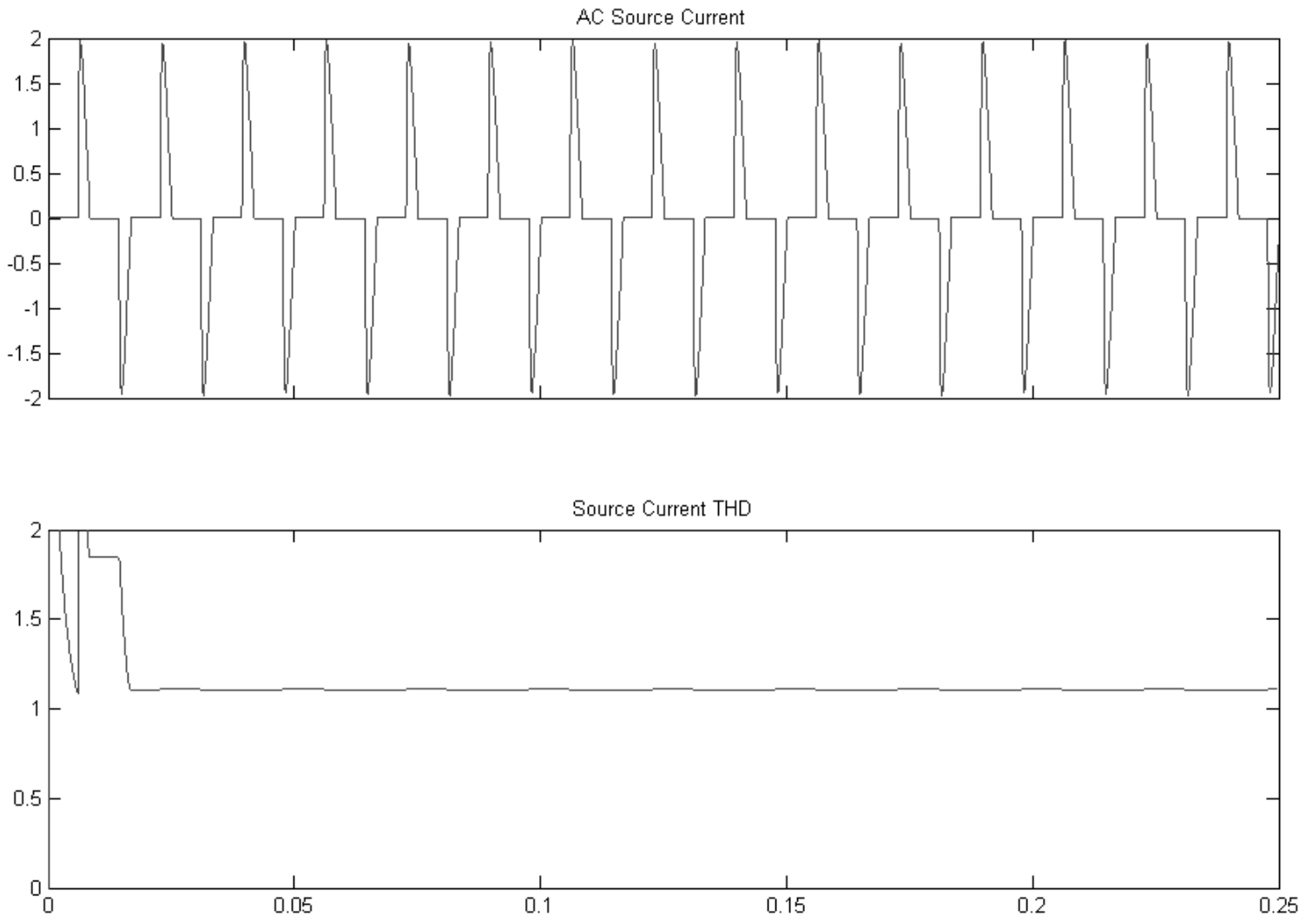

Figure 4.8: Load Without Line Conditioner

for in the inverter current. Pulses in the output current of the line conditioner correspond with the load pulses, showing that the synchronous link is functioning by directing harmonic currents to the inverter. Furthermore, the DC capacitor voltage remains relatively stable despite the periodic surges on the line conditioner output current.

These results are similar to those presented in [29], indicating a successful reproduction of the active power quality conditioner. In addition, the simulation model was simplified somewhat from Kumar's model, eliminating the reactive current monitor and DC current feedback loop.

\subsection{Digital Control}

Once the analog simulation was working properly, the next step was to modify the controller and make it a digital system. The goal was to create a control system that could be implemented on the same PIC microcontroller as the motor controller, eliminating the need for additional 


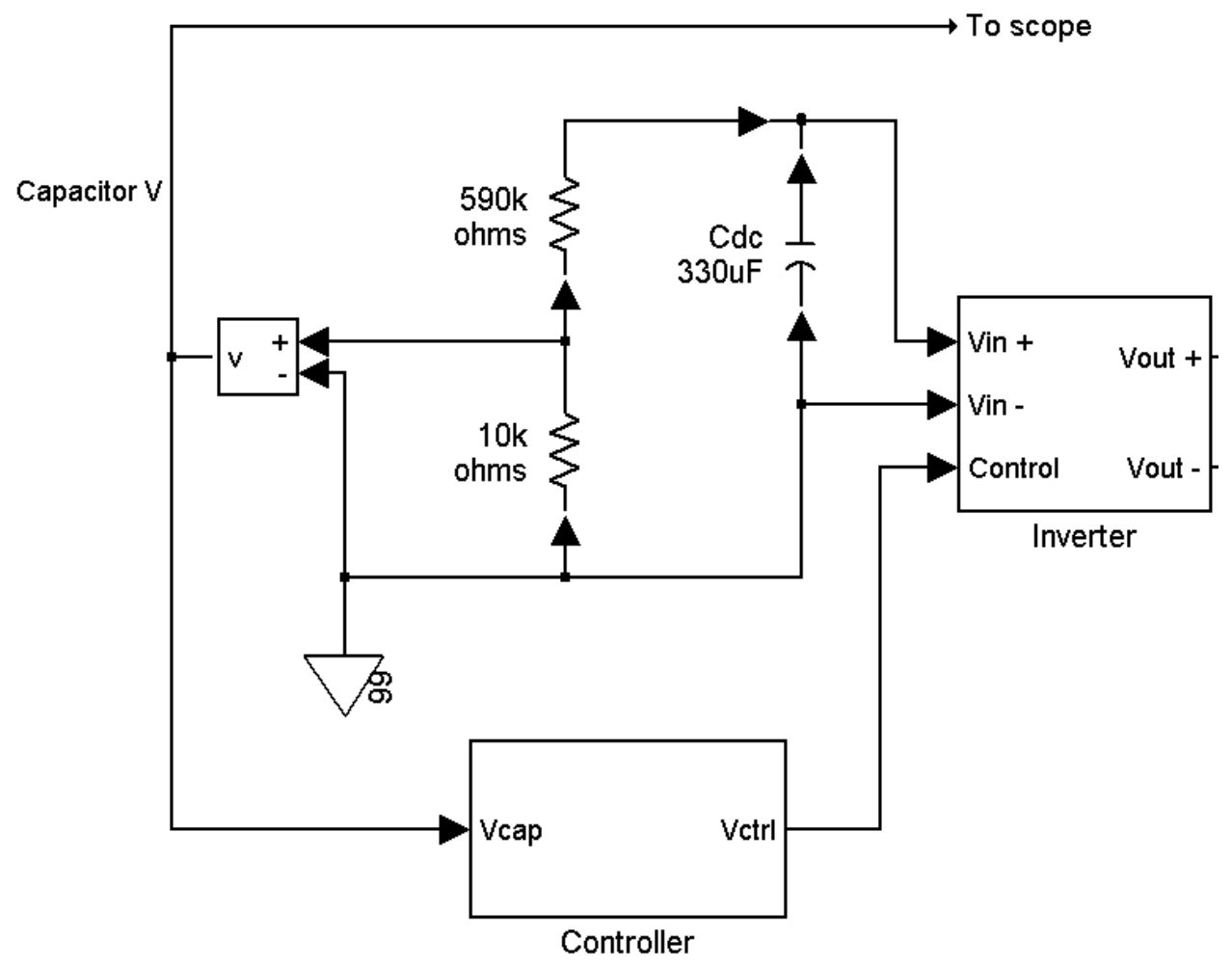

Figure 4.9: Voltage Divider for Digital Controller

analog control hardware.

The top-level block diagram of the digital system simulation model differs very little from its analog counterpart; in fact, the most significant change was the addition of a voltage divider across the DC capacitor. This alteration, shown in Figure 4.9, was made to account for the ADC module found in the PIC microcontroller, which performs conversions in the range $0 \mathrm{~V}$ to $5 \mathrm{~V}$ unless supplied with additional reference voltages. The resistance values shown will provide an output range from $0 \mathrm{~V}$ to $5 \mathrm{~V}$ corresponding with a $\mathrm{DC}$ capacitor voltage of $0 \mathrm{~V}$ to $300 \mathrm{~V}$.

All other changes to the model are internal to the controller and inverter blocks. The new controller is shown in Figure 4.10, and this illustration also provides insight into the change made to the inverter. It can be readily observed that the controller now contains the PWM components, which have been removed from the inverter block; no other alterations were made to the inverter. Another significant change to be noted in the controller block is the lack of an input port for the AC line voltage.

A saturation block connected to the input of the controller ensures that the voltage signal is 


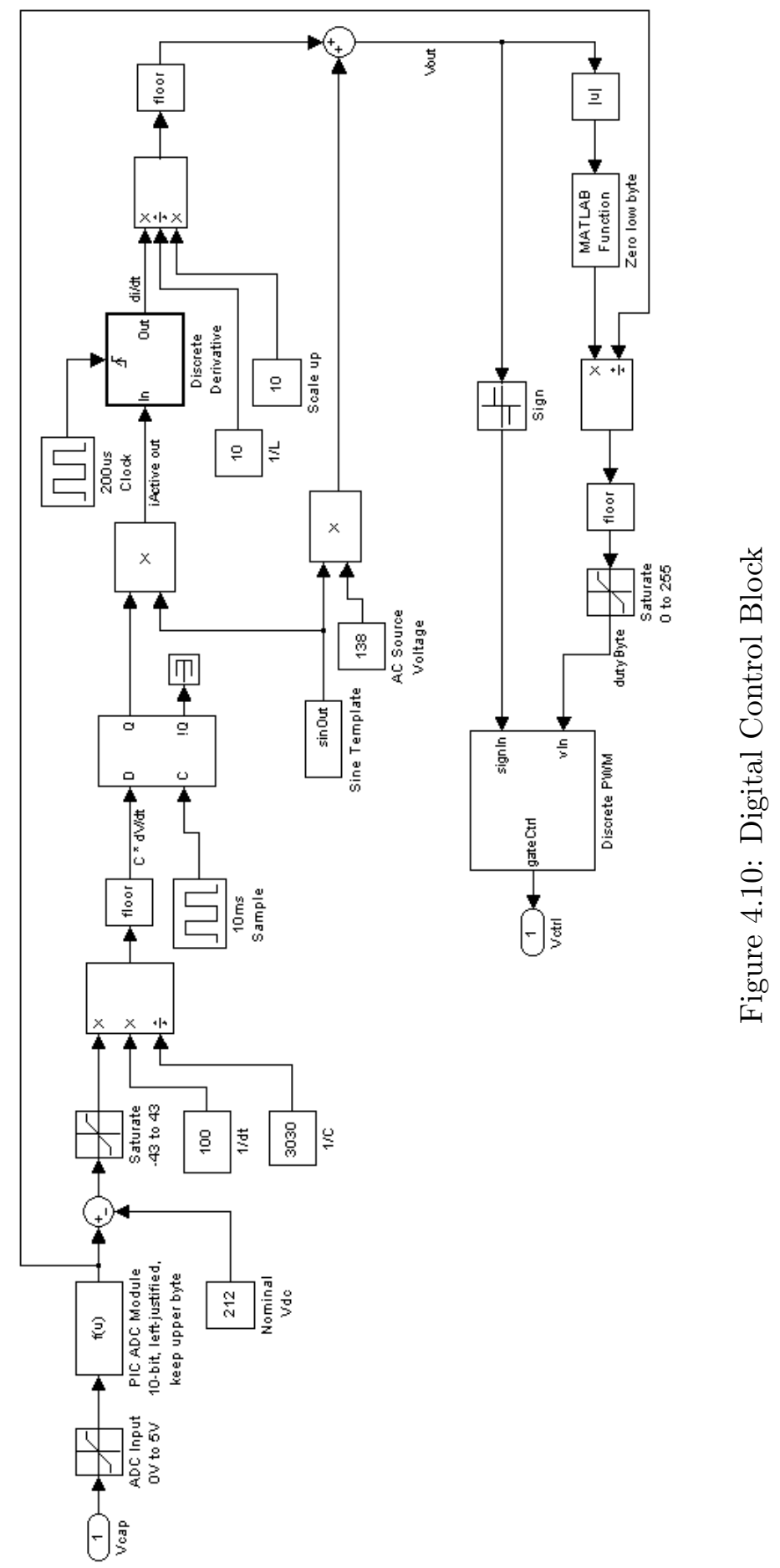




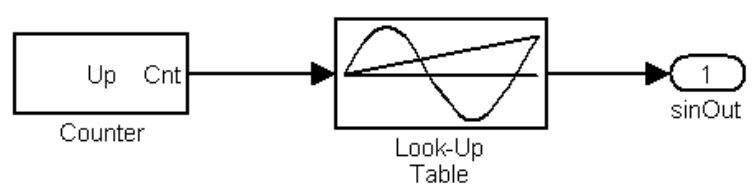

Figure 4.11: Lookup-Based Sine Wave Template

between $0 \mathrm{~V}$ and $5 \mathrm{~V}$. This value is then passed to a function block that emulates the PIC ADC module by converting the voltage signal to a number. The PIC is capable of producing a digital value with 10-bit resolution that can be either left- or right-justified in a two-byte memory block. For this application, the value was left justified and the two least significant bits were dropped to produce a single-byte value. The formula used to perform this operation is floor (floor (u/5 * 1023) / 4), where u represents the input signal.

With the capacitor voltage captured, it is compared to its nominal value of $250 \mathrm{~V}$. The constant representing the nominal voltage and the limits of the saturation block that follows the comparison have been changed to reflect the byte representations of their values. To calculate the DC capacitor current, values for $\frac{1}{d t}$ and $\frac{1}{C}$ are used in conjunction with the appropriately altered multiply/divide block. This approach was taken to avoid the use of floating-point numbers in the calculation, as integer math is much more efficient on the class of microcontrollers this application is targeted for. The result of the $C * \frac{d V}{d t}$ calculation is passed through a floor block before reaching the D-latch to ensure an integer value. It should also be noted that the sampling frequency in the digital controller is $100 \mathrm{~Hz}$ as opposed to $240 \mathrm{~Hz}$ in the analog version.

Figure 4.11 shows the internals of the sine wave template block, the output of which is multiplied by the latched capacitor current. A free-running counter is used to reference a value in a lookup table that contains integer values for a sine wave with 255 magnitude. There are 500 values in the lookup table, which covers a time span of $100 \mathrm{~ms}$. Choosing $100 \mathrm{~ms}$ as the time span of the table helps reduce timing errors, as that period contains six full cycles of a $60 \mathrm{~Hz}$ signal. The values were generated by multiplying the output of a custom-written MATLAB function, genSineWaveTable, by 255 and rounding to the nearest integer. The full source of genSineWaveTable.m can be found in Section A.2.3.

The result of multiplying the latched DC capacitor current with the sine template is passed to the triggered subsystem detailed in Figure 4.12. This block is triggered every $200 \mu$ s, which corresponds with the amount of time between values in the sine wave template lookup table. A memory block stores the last input state, which is subtracted from the current input state and 


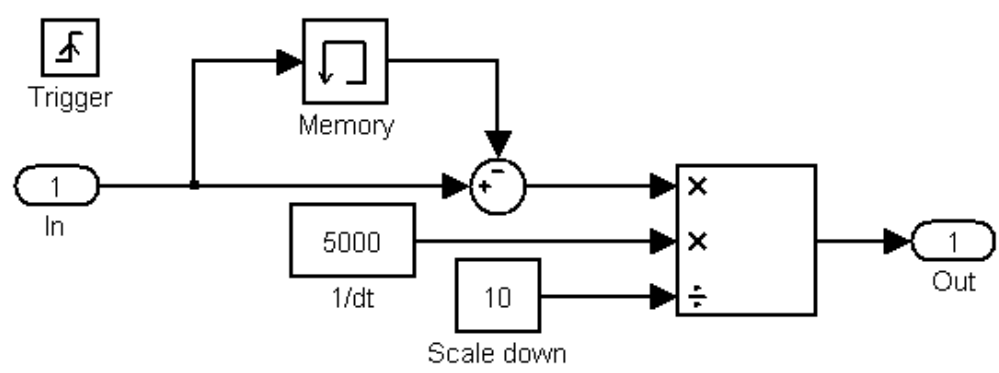

Figure 4.12: Discrete Derivative Subsystem

passed to a multiplier block. The other inputs to the multiplier are the multiplicative inverse of the time step and a scale factor. The scale factor was used to ensure that the result could be represented by two bytes.

Using the output of the discrete derivative, the inverter output voltage was calculated. The signal is divided by the inverse of the link reactor inductance to produce the voltage across the link reactor. This value is then multiplied by the scale factor to counteract the scaling operation performed in the discrete derivative block. A floor block maintains an integer value, then a sum block combines the link reactor voltage with the AC source voltage. However, the AC line voltage is simulated rather than measured. This is achieved by multiplying the sine wave template by a constant that represents the magnitude of the AC voltage.

A sign block passes the sign of the desired output voltage to the PWM module. The voltage signal is also passed through an absolute value block, then has its lower byte zeroed via a bitwise AND operation with the hexadecimal value 0xFF00. The zeroing operation is performed using the built-in MATLAB function bitand, which must be contained in a MATLAB function block. Removing the lower byte causes the following division by the capacitor voltage to result in a single-byte value, although floor and saturation blocks are included as a precaution, and the output is passed to the discrete PWM block.

The standard Simulink PWM generator block was removed and replaced with the block detailed in Figure 4.13. The first input is the sign of the desired voltage that, because of the implementation of the sign block, is -1 for negative numbers, 1 for positive numbers, and 0 for zero. Before applying the gate logic, this signal needed to be changed to a bit value. The "Convert to bit" block produces 0 for negative and 1 for positive.

Before generating any pulses, it is necessary to perform a conversion on the input voltage value, shown as the dutyByte signal in Figure 4.10 . This signal value ranges from 0 to 255, 


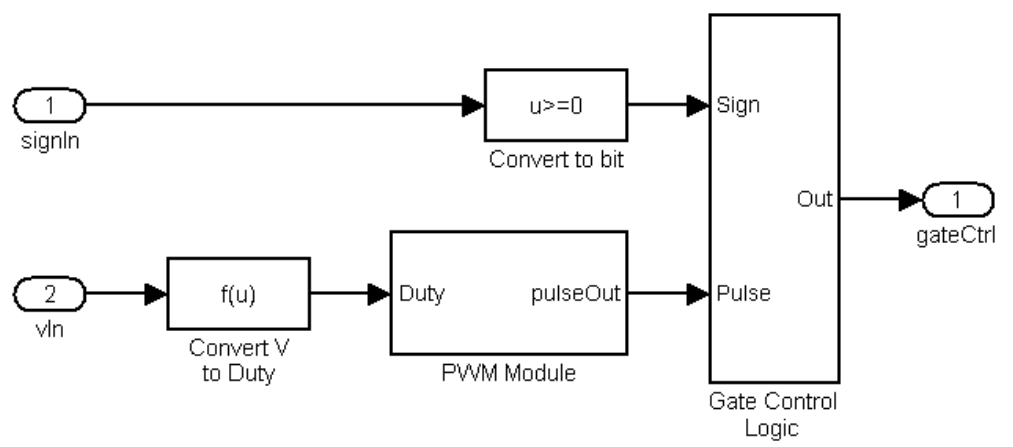

Figure 4.13: Discrete PWM Block

and represents the voltage to be output by the inverter. The PWM module on the PIC18F452 microcontroller uses a 10-bit duty cycle, so this byte value must be scaled up to produce the appropriate magnitude. While a maximum duty cycle value of 800 was possible in this case, it was found that the range 0 to 372 provided a more stable inverter output.

Normally, this conversion would be performed by Equation 4.1 however, the PIC microcontroller does not have a built-in floating-point processor and integer division would result in a great loss of accuracy. To be implemented more efficiently on the PIC, Equation 4.2 was derived as shown below. This expression can be programmed using only simple addition and bit-shift operations, which are performed quickly by the arithmetic unit on the PIC. The resulting duty value is passed to the PWM module block for pulse generation.

$$
\begin{aligned}
\text { duty } & =\text { voltage } \times \frac{372}{256} \\
& =\text { voltage } \times\left(1+\frac{116}{256}\right) \\
& =\text { voltage } \times\left(1+\frac{128}{256}-\frac{8}{256}-\frac{4}{256}\right) \\
& =\text { voltage } \times\left(1+\frac{1}{2}-\frac{1}{32}-\frac{1}{64}\right) \\
\text { duty } & \approx \text { voltage }+\frac{\text { voltage }}{2}-\frac{\text { voltage }}{16}
\end{aligned}
$$

Figure 4.14 shows the internals of the PWM module block. The calculated duty cycle is compared to the output of a repeating sequence block. The sequence is defined by the points $(0$, 1) and $\left(199.75^{*} 10^{6}, 800\right)$, which represents a counter that increments every $250 \mathrm{~ns}$ and resets every $200 \mu \mathrm{s}$. The use of a counter block would have forced a simulation time step every 250ns, slowing 


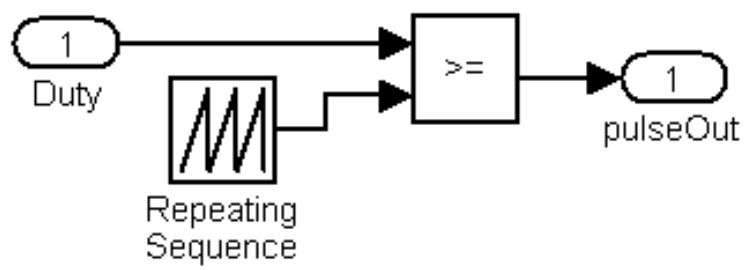

Figure 4.14: Discrete PWM Pulse Generator

\begin{tabular}{c|c||c|c|c|c} 
Pulse & Sign & G1 & G2 & G3 & G4 \\
\hline 1 & 0 & 0 & 1 & 1 & 0 \\
1 & 1 & 1 & 0 & 0 & 1 \\
0 & - & 1 & 0 & 1 & 0
\end{tabular}

Table 4.1: PWM Gate Logic Truth Table

the simulation to an unreasonable level, so the repeating sequence was used as a replacement. When the duty value at the input port is greater than or equal to the value of the counting sequence, a pulse is generated.

The pulse and sign are passed to a combinational logic block governed by the truth table listed in Table 4.1. The outputs G1 - G4 are produced by Equations 4.3-4.6. These outputs are multiplexed and sent to the MOSFET bridge, where they are used as gate signals.

$$
\begin{aligned}
G 1 & =\text { Sign }+\overline{\text { Pulse }} \\
G 2 & =\overline{\text { Sign } \bullet \text { Pulse }} \\
G 3 & =\overline{\text { Sign }}+\overline{\text { Pulse }} \\
G 4 & =\text { Sign } \bullet \text { Pulse }
\end{aligned}
$$

\subsubsection{Results}

Scope traces from the digital line conditioner simulation are shown in Figure 4.15, and are comparable to the analog results previously presented. This simulation was run with the same load as its analog counterpart, but for a slightly longer time interval. See Section 4.2 .2 for more details about the analog line conditioner simulation results.

A key difference between the analog and digital results is the longer amount of time needed to charge the DC capacitor under digital control. When the load is switched on at $100 \mathrm{~ms}$, the 


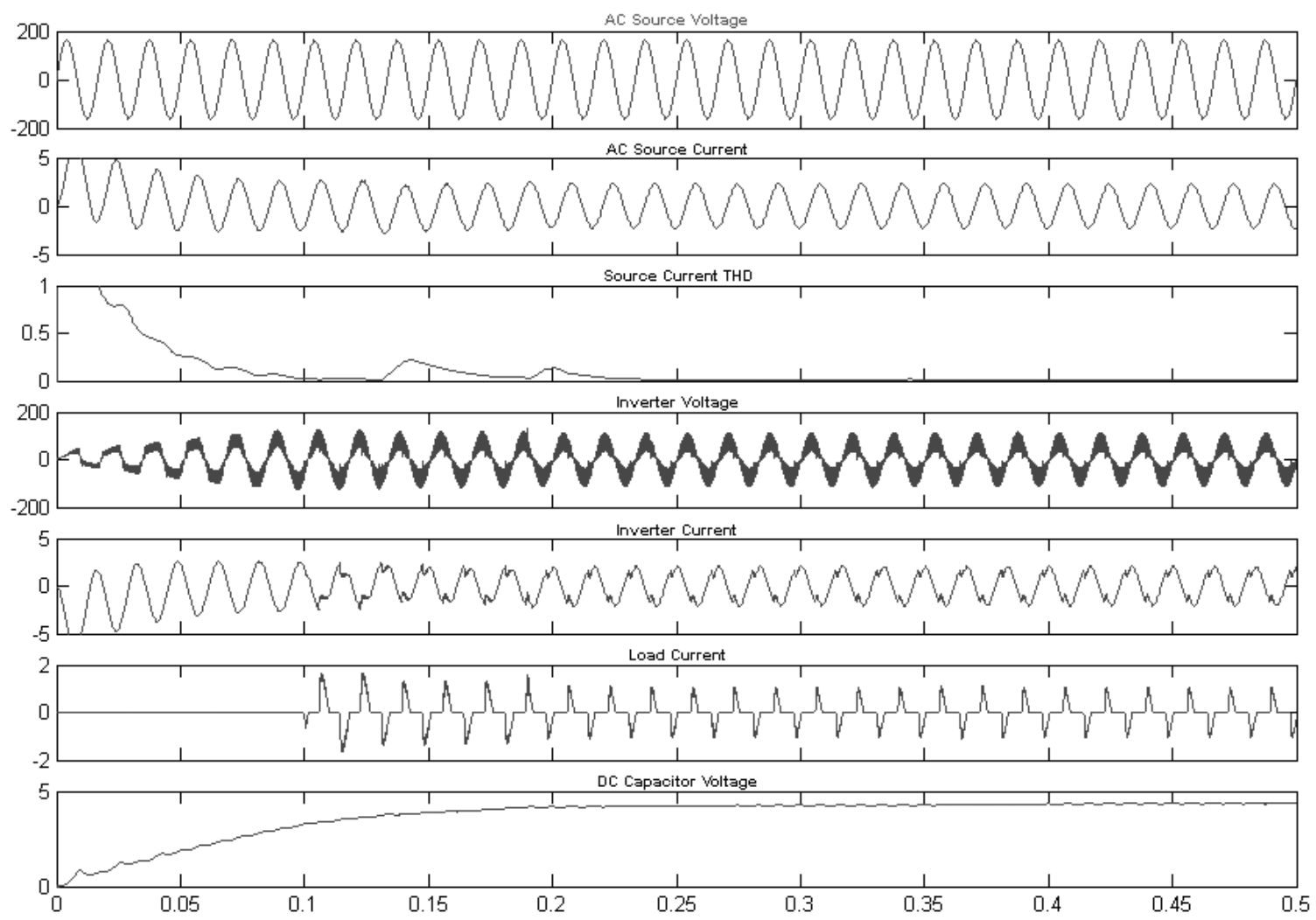

Figure 4.15: Digital Control Simulation Results

capacitor has not yet reached its nominal voltage, and the load current waveform has a much higher amplitude than was shown in Figure 4.7. The current drawn from the AC source is also higher as a result.

Figure 4.15 shows that the line conditioner does continue to charge the capacitor even while the load is active. The RMS value of the line current is $1.68 \mathrm{~A}$ between $0.15 \mathrm{~s}$ and $0.2 \mathrm{~s}$, and $1.62 \mathrm{~A}$ between $0.2 \mathrm{~s}$ and $0.25 \mathrm{~s}$. Likewise, the RMS value of the load current drops from $518 \mathrm{~mA}$ to $406 \mathrm{~mA}$ over the same intervals. From $0.25 \mathrm{~s}$ to $0.5 \mathrm{~s}$, the line current stabilizes at $1.61 \mathrm{~A}$ RMS and the load current is further reduced to $393 \mathrm{~mA}$.

The harmonic distortion curve is another indicator that the system does not reach a steady state in the first $0.25 \mathrm{~s}$ of the simulation. Two pulses can be observed after $100 \mathrm{~ms}$ in the graph in Figure 4.15, though the second is considerably smaller than the first. Even with these instabilities, the digital controller manages to outperform the analog controller, achieving RMS THD values of $8.82 \%$ between $0.15 \mathrm{~s}$ and $0.2 \mathrm{~s}$ and $5.37 \%$ between $0.2 \mathrm{~s}$ and $0.25 \mathrm{~s}$. Beyond $0.25 \mathrm{~s}$, the harmonic 
disortion in the line current further drops to $1.10 \%$ RMS.

\subsection{Complete System Simulation}

With the successful development of a microcontroller-based line conditioner, the motor controller model was added and the complete system simulated. The block diagram is shown in Figure 4.16. Though the line conditioner controller and motor controller are combined into one block, no changes were made to the components; they were simply grouped together. Current analyzer and inverting amplifier blocks were necessarily added as part of the motor controller, and are described in detail in Section 3.5.2.

\subsubsection{Results}

The simulation was run with the same loads and time interval used in the motor controller subsystem simulation (see Section 3.5.4). Table 4.2 shows a comparison of the complete system results and the uncontrolled load measurements. Note that the resistive load was excluded from this test: the primary goal of adding the line conditioner was to reduce harmonics induced by the motor controller, and it can be seen in Section 3.5.5 that no additional THD was created with the $100 \Omega$ load. Differences were calculated by subtracting the conditioned measurements from the uncontrolled values, so a negative number indicates an increase. Selected waveforms from the simulation are presented in Figures 4.17-4.19.

The table shows a slight increase in harmonic distortion at all load levels, but the average rise was only $3.27 \%$. Comparing the highest level of harmonic distortion with conditioning, at 5.56\%, to the lowest THD value recorded with the motor controller alone, 22.97\% (see Table 3.4), shows that harmonics generated by switching the load triac are greatly reduced by the line conditioner. The dramatic decrease in harmonic distortion is illustrated in Figure 4.20

Aside from a slight anomoly under the 6 inch-ounce load, the real power consumed by the system with power quality conditioning remains relatively constant regardless of the load, as does the reactive power. It would appear that the line conditioner dominates the load in terms of power requirements.

Reductions in real power consumption between the uncontrolled and conditioned systems can be observed at all load levels; however, slight increases in current draw and significant increases in reactive power are also shown in Table 4.2. Figure 4.21 contains plots of both the real and 


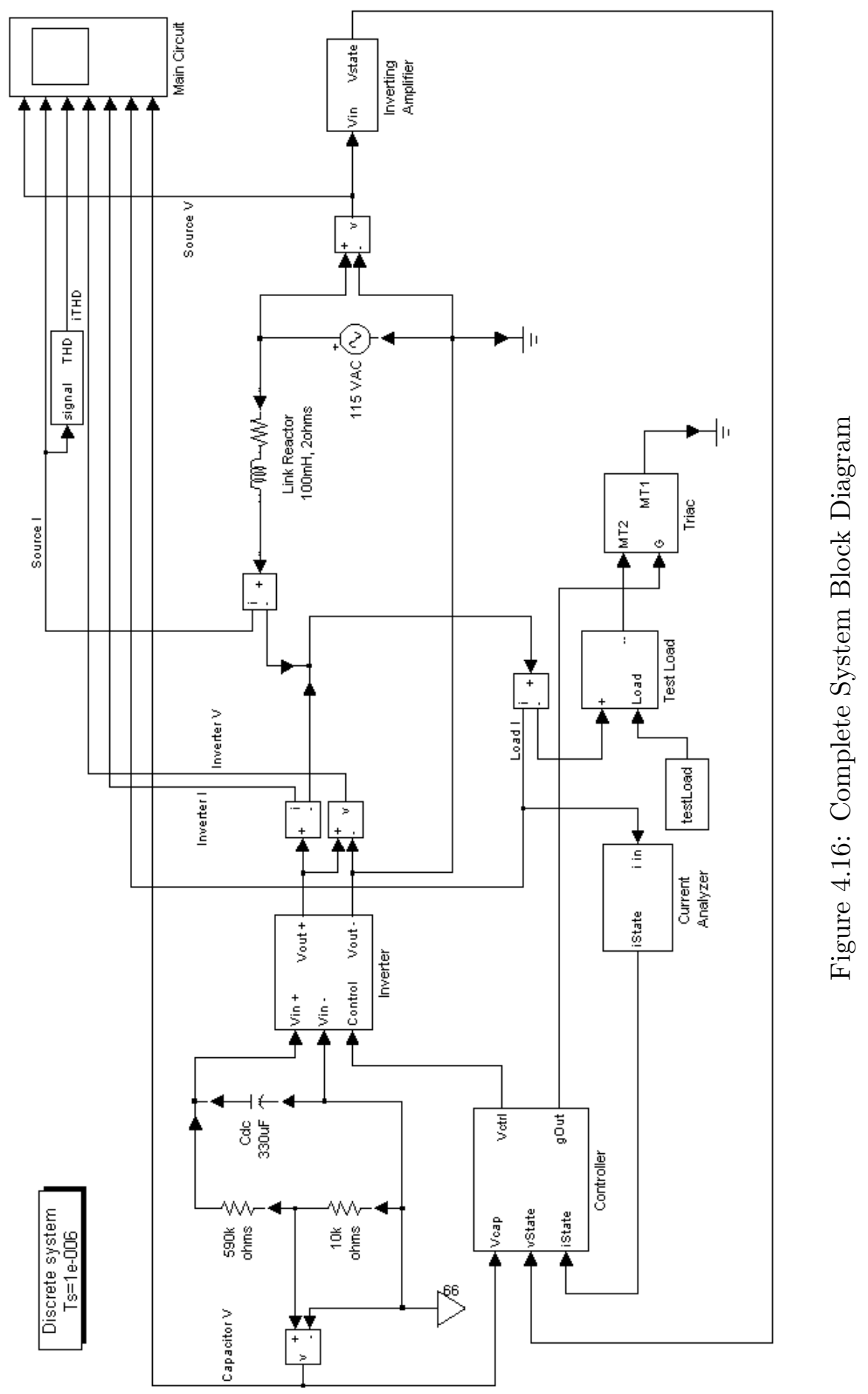




\begin{tabular}{c|r||c|c|c|c}
\multicolumn{2}{c|}{} & $\mathrm{I}_{R M S}(\mathrm{~A})$ & $\mathrm{I}_{T H D}(\%)$ & $\mathrm{P}(\mathrm{W})$ & $\mathrm{Q}(\mathrm{VAR})$ \\
\hline \hline No load & Uncontrolled & 1.2176 & 0.46 & 34.1416 & 135.8551 \\
\cline { 2 - 6 } & Conditioned & 1.6258 & 2.54 & 22.5845 & 185.3546 \\
\cline { 2 - 6 } & Difference & -0.4082 & -2.08 & 11.5571 & -49.4995 \\
\hline \hline 6 in.-oz. & Uncontrolled & 1.2108 & 0.46 & 47.4202 & 130.9964 \\
\cline { 2 - 6 } & Conditioned & 1.6147 & 5.56 & 19.3923 & 183.6851 \\
\cline { 2 - 6 } & Difference & -0.4039 & -5.1 & 28.0279 & -52.6887 \\
\hline \hline 7 in.-oz. & Uncontrolled & 1.2205 & 0.46 & 56.3312 & 128.6632 \\
\cline { 2 - 6 } & Conditioned & 1.6229 & 0.81 & 25.3928 & 184.6621 \\
\cline { 2 - 6 } & Difference & -0.4024 & -0.35 & 30.9384 & -55.9989 \\
\hline \hline 8 in.-oz. & Uncontrolled & 1.3286 & 0.46 & 90.1746 & 123.5144 \\
\cline { 2 - 6 } & Conditioned & 1.6273 & 0.97 & 25.7228 & 185.1328 \\
\cline { 2 - 6 } & Difference & -0.2987 & -0.51 & 64.4518 & -61.6184 \\
\hline \hline 9 in.-oz. & Uncontrolled & 1.3558 & 0.46 & 95.2645 & 123.6184 \\
\cline { 2 - 6 } & Conditioned & 1.6132 & 0.95 & 26.3338 & 183.3946 \\
\cline { 2 - 6 } & Difference & -0.2574 & -0.49 & 68.9307 & -59.7762 \\
\hline \hline 10 in.-oz. & Uncontrolled & 1.4082 & 0.46 & 106.2242 & 122.4464 \\
\cline { 2 - 6 } & Conditioned & 1.6251 & 0.95 & 25.7319 & 185.015 \\
\cline { 2 - 6 } & Difference & -0.2169 & -0.49 & 80.4923 & -62.5686 \\
\hline \hline
\end{tabular}

Table 4.2: Complete System Simulation Results 

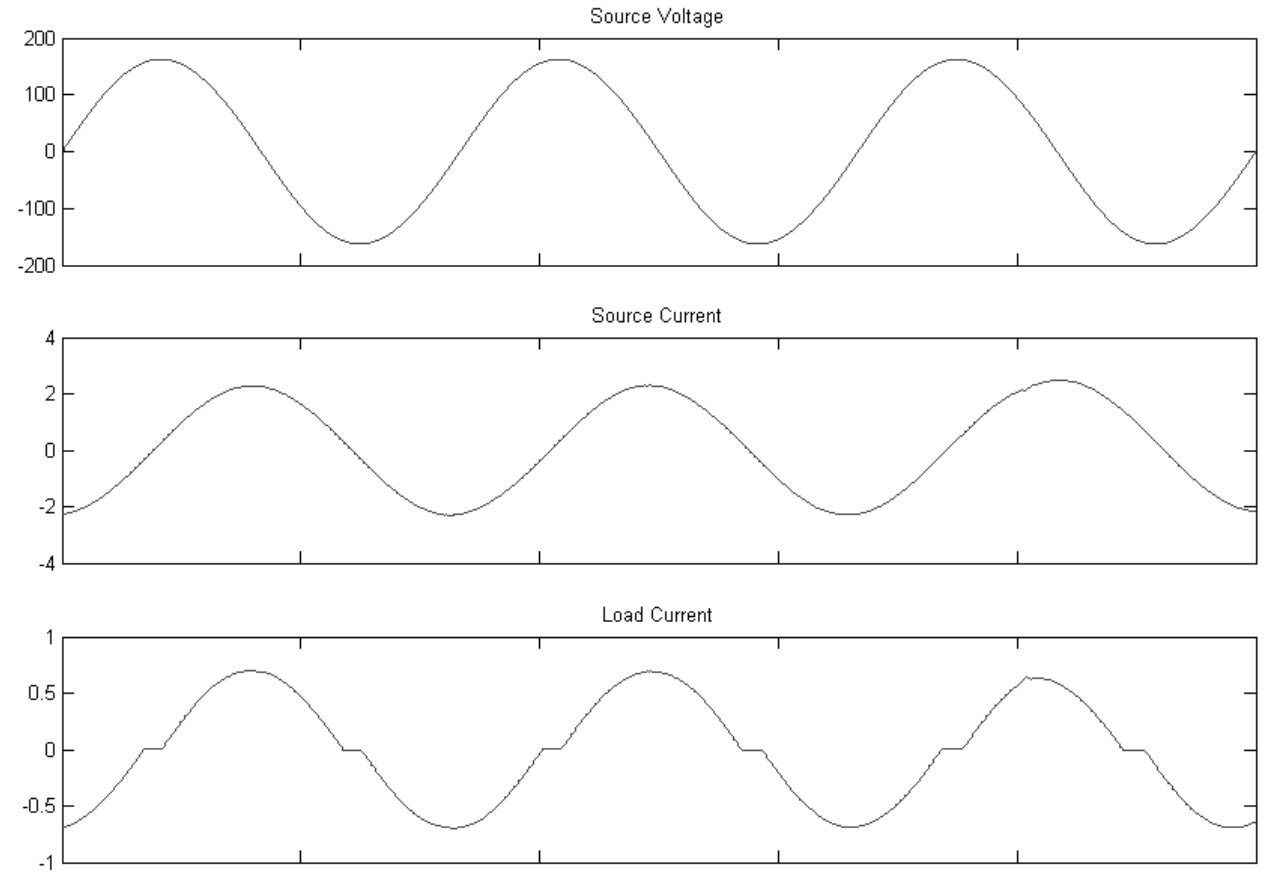

Figure 4.17: Complete System Results, No Load
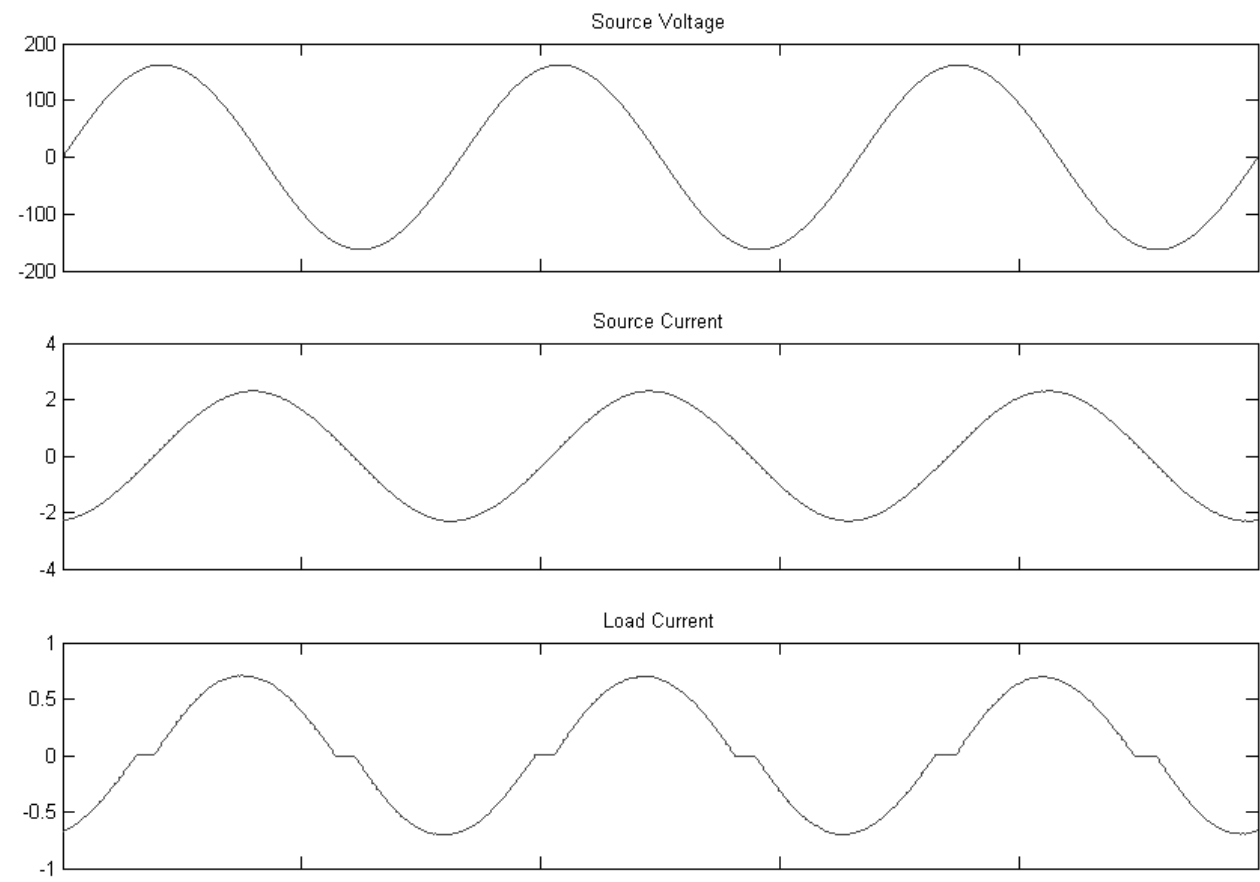

Figure 4.18: Complete System Results, 7 in.-oz. Load 

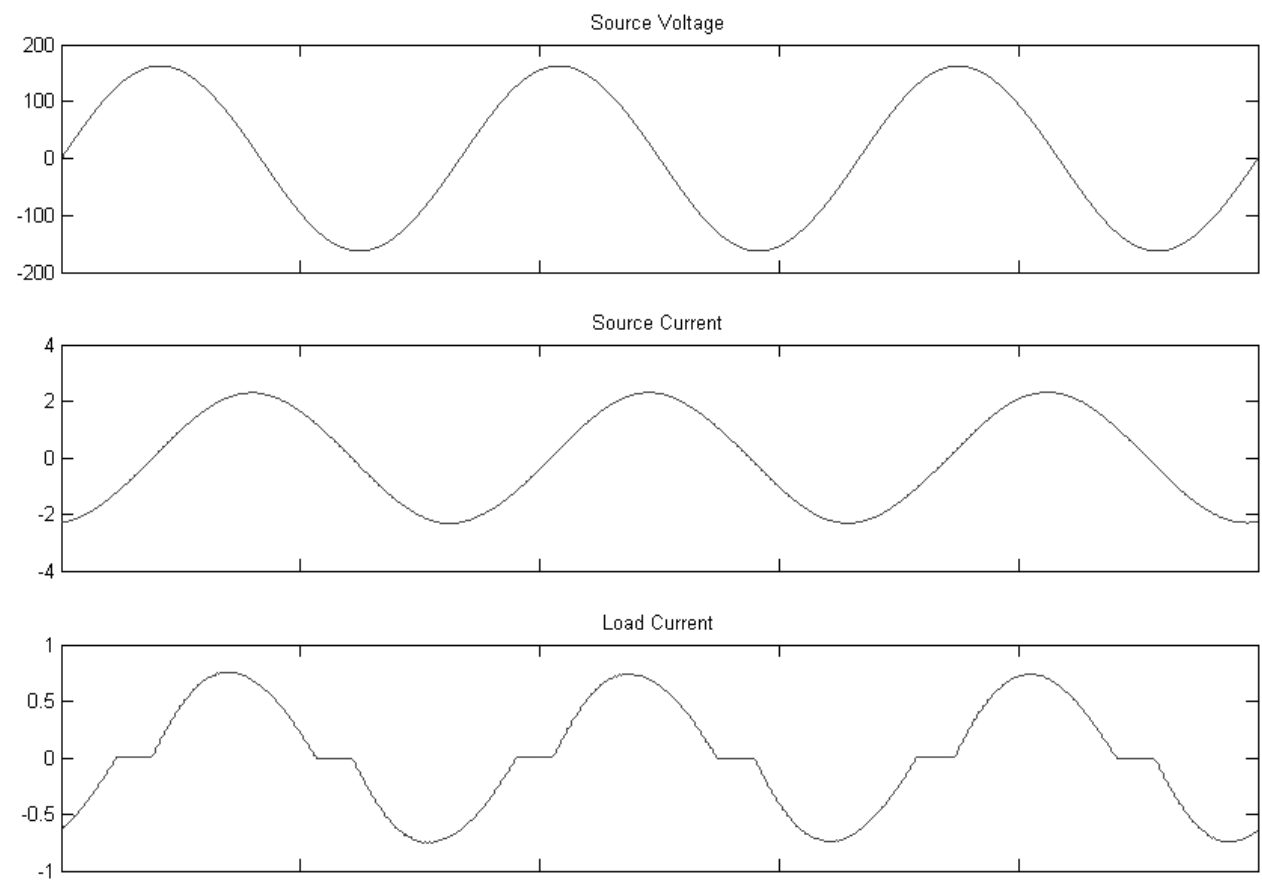

Figure 4.19: Complete System Results, 10 in.-oz. Load

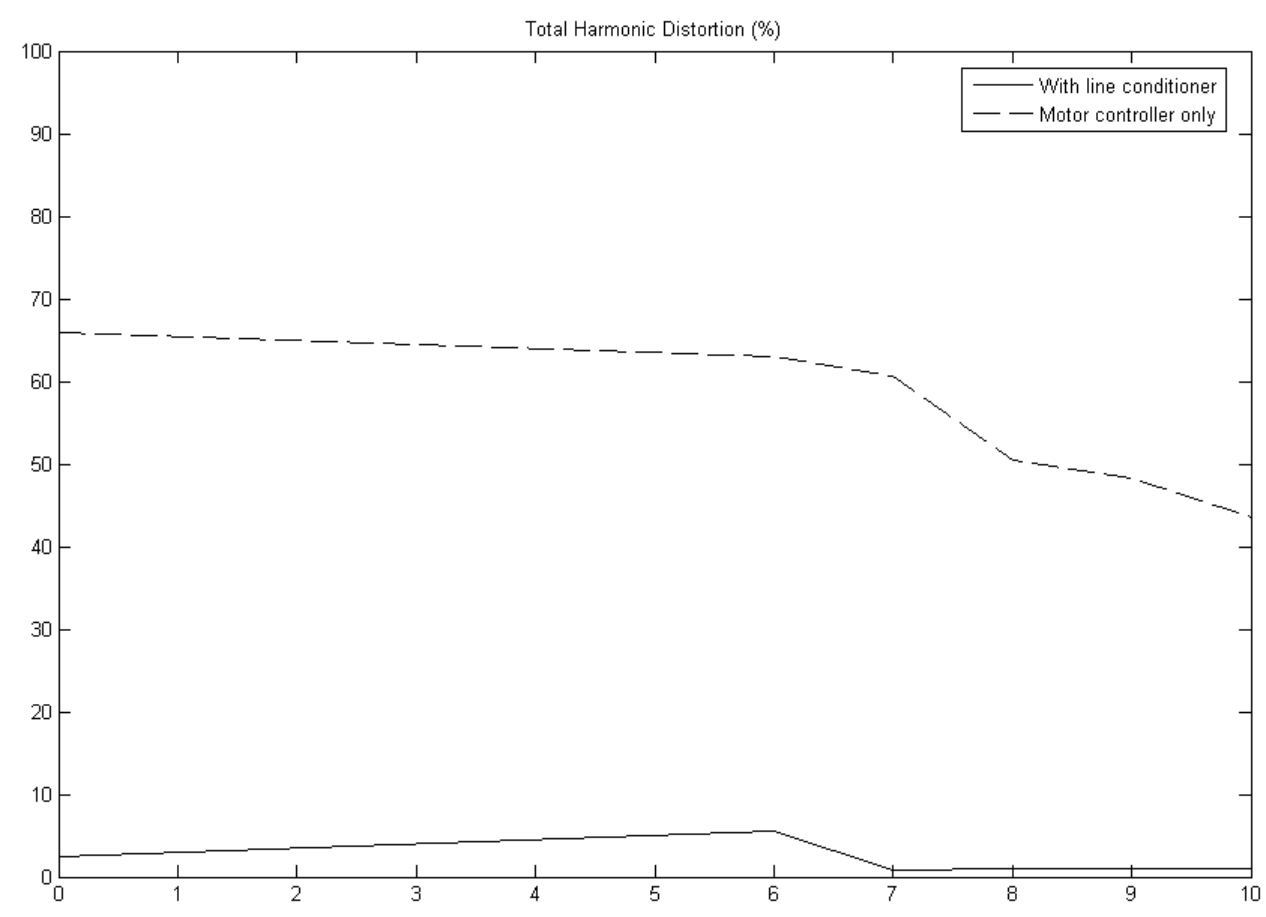

Figure 4.20: Total Harmonic Distortion Versus Load 

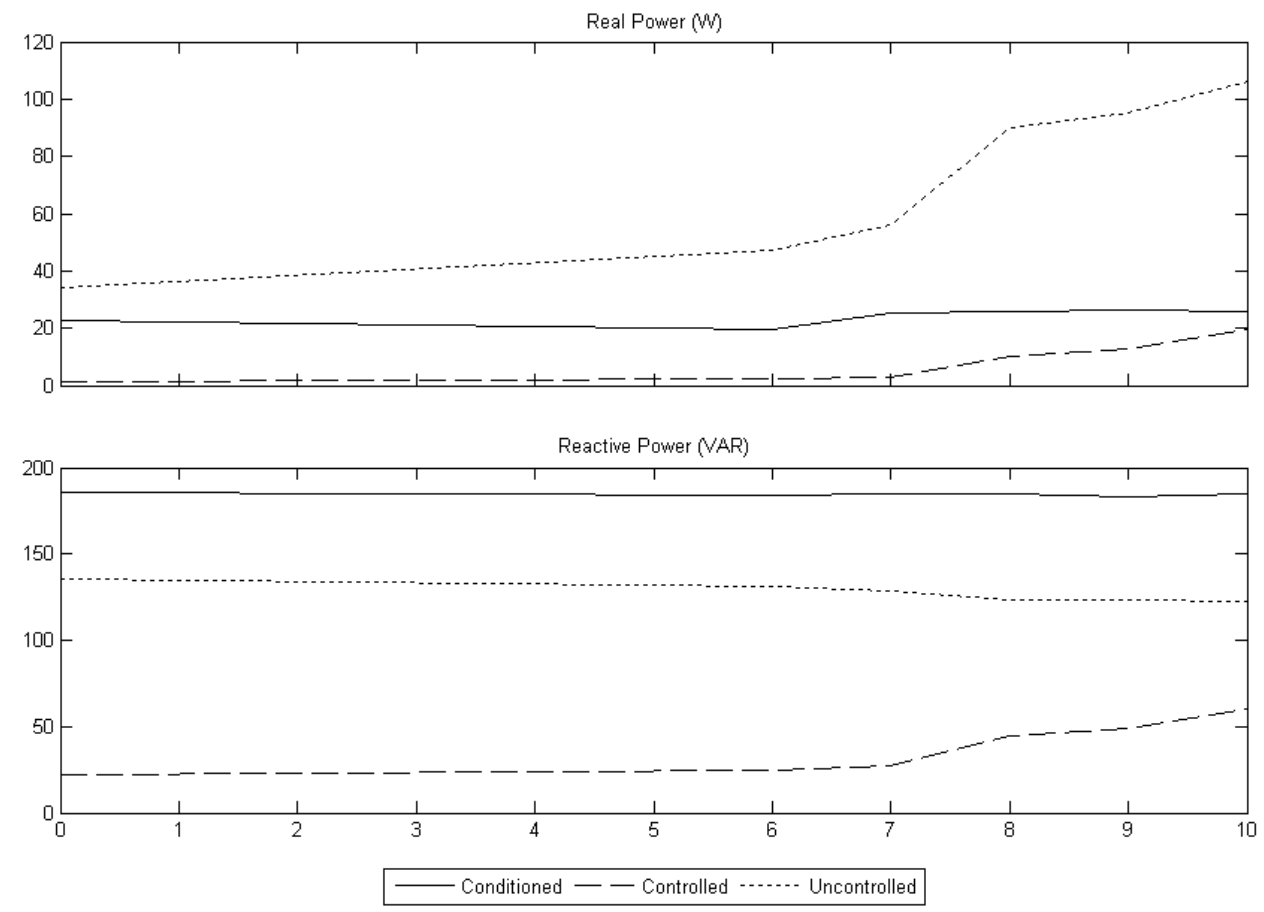

Figure 4.21: Real and Reactive Power Versus Load

reactive power consumed by the system with no controller (uncontrolled), the motor controller only (controlled), and the motor controller with line conditioner (conditioned). While the conditioned system does consume more real power than the controlled system, a savings over the uncontrolled load is still achieved. The reactive power curves confirm that the line conditioner system actually consumes more VARs than the motor alone.

In order to better compare the data, the total power $S=P+j Q$ was converted to polar form for each load. Results of these calculations are shown in Table 4.3 , where it is shown that the line conditioner actually increases the amount of total power drawn.

The increase in consumed VARs can be attributed to the link reactor, which introduces a relatively large inductance into the system. This is evidenced by the high phase angles recorded in Table 4.3, which are all greater than 80 degrees. Again, the total power measures remain relatively constant for every load level, indicating that the line conditioner provides the bulk of the impedance seen by the source. A larger load, then, may produce different results.

Despite the increase in reactive power, the combined system does result in a significant decrease in real power consumption. In addition, the total harmonic distortion of the $\mathrm{AC}$ line is 


\begin{tabular}{c||c|c} 
Load (in.-oz.) & Uncontrolled & Conditioned \\
\hline \hline 0 & $140.08 \angle 75.89$ & $186.73 \angle 83.05$ \\
\hline 6 & $139.32 \angle 70.10$ & $184.71 \angle 83.97$ \\
\hline 7 & $140.45 \angle 66.36$ & $186.40 \angle 82.17$ \\
\hline 8 & $152.93 \angle 53.87$ & $186.91 \angle 82.09$ \\
\hline 9 & $156.07 \angle 52.38$ & $185.28 \angle 81.83$ \\
\hline 10 & $162.10 \angle 49.06$ & $186.80 \angle 82.08$
\end{tabular}

Table 4.3: Total Power Consumption

not significantly affected. Therefore, it can be concluded that the simulated implementation of the motor controller with line conditioning on a microcontroller was successful. 


\section{Chapter 5}

\section{Conclusion}

\subsection{Summary}

This thesis presented a system for reducing the power consumed by an induction motor while addressing the issue of harmonic distortion. Existing designs were researched, duplicated, modified as needed, and combined to be controlled by a single-chip microcontroller.

The motor controller uses a triac to chop the input voltage and current to the load, effectively reducing its power consumption. A microcontroller accepts the sign of the AC line voltage and the triac gate voltage as inputs, and determines the phase angle of the load based on the time between voltage and current zero-crossings. Based on this calculation, pulses are sent to the triac, switching it to a conductive state and providing power to the motor as needed.

Line conditioning is achieved through the use of a synchronous link. A DC capacitor stores energy supplied by the AC line, which is inverted to produce a secondary sinusoidal voltage source. A link reactor is inserted between the AC line and the load that allows the inverter to provide any harmonic components of the load current. The controller monitors the DC voltage, calculates the required output voltage, and provides pulse-width modulated signals to the inverter.

A hardware prototype of the triac-based motor controller was built using a PIC18F452 microcontoller. Test results showed an average decrease of $23.2 \%$ in real power consumption, with a maximum reduction of $56.3 \%$. This maximum occurred when the motor was not loaded, and resulted in a savings of 19.24 Watts. However, the chopping of the load current waveform induced by switching the triac caused an average THD increase of $426.6 \%$.

The motor controller was then modeled using Simulink, and a test load was created using data from the hardware test. Data from the simulation showed the same trends as were observed 
in the empirical results: reductions in current draw and real power consumption are greater under light load conditions, as is the harmonic distortion.

The line conditioner was simulated based on an existing design, and after verifying that it functioned correctly with analog control elements, the subsystem was modified to model implementation on a microcontroller. Steps taken to accomodate digital functionality included changing mathematical operations from floating-point to integer expressions, scaling to one- or two-byte values when possible, and using a lookup table to generate values for a sine wave reference. Simulation results showed THD values as low as $1.1 \%$ for a switching load similar to the one used for the motor controller simulation.

After showing that the digital line conditioner effectively reduced THD, it was combined with the motor controller model and the entire system was successfully tested. The maximum recorded harmonic distortion from the simulation was only $5.56 \%$, proving that the conditioner effectively compensates for triac-induced chopping of the current waveform. However, a large increase in reactive power resulted in the total power consumption of the system to increase. Despite this, real power consumption is decreased when compared to the uncontrolled load and THD is kept under control, so the project was considered a success.

Overall, the goals of the research were met. Tests of a triac-based motor controller verified both the power-saving characteristics and harmonic distortion problem of such a system. The addition of a line conditioner improved the power quality, and the complete system consumed less active power than the uncontrolled motor load. It was shown that it is feasible to implement all control logic for the system on a low-cost single-chip microcontroller, resulting in a simplified circuit. Though an increase in total power was observed, the successful reduction of harmonic distortion and active power consumption is an important result that shows the usefulness of this research.

\subsection{Practicality}

Results of the complete system simulation show that although real power consumed by the load is decreased, the line conditioner increases the total power draw from the AC line. This raises the question of how practical an implementation of the system would be.

The link reactor used by the line conditioner was the primary cause of the total power increase, requiring a large amount of reactive VARs to be consumed by the system. Additional power quality conditioning, such as a VAR compensator, could balance out the reactive power draw. 
However, the motor used for hardware testing, which also served as the basis of the simulated load, was representative of what could be found in household appliances. As residential power customers do not usually employ VAR compensators or capacitor banks, the system in its current state would probably not be suitable for this market.

Larger loads could potentially benefit from the system, as the line conditioner would be less likely to be the dominant impedance. In addition, heavy industrial loads may also benefit from the use of reactive compensators, balancing the power factor. As many of these loads use three-phase power, additional research and design efforts would be needed to adapt the microcontroller-based motor controller with line conditioning to the industrial market.

\subsection{Future Work}

Though the simulation of the motor controller with line conditioning presented in this work was designed to account for the limitations of a low-power microcontroller, a better way to ensure an accurate simulation would be to use an emulator. Emulators provide hardware functionality in software, so PIC C code could be written and used for the controller in simulation. Taking this one step further, a hardware-in-the-loop simulation could run code on a real microcontroller, exchanging input and output signals with the simulation. Of course, both emulation and hardware-in-the-loop would require an interface to be created in Simulink (or any other simulation environment).

The proposed extensions to the simulation would lead naturally to the construction of a hardware prototype. Extensive research on components would need to be performed to ensure that power ratings are not exceeded. Besides testing the overall effectiveness of the motor controller and line conditioner system, a side-by-side comparison with other devices, such as the Power Planner, could be undertaken. An additional study into the electromagnetic interference generated by the hardware would also be of interest.

Combining the system presented in this thesis with another device such as the personal static var compensator described in [17] could result in a multi-functional power regulation device. While it is doubtful that there is a "miracle drug" solution to solve all possible power quality issues, the power of microcontrollers and digital signal processing chips could warrant the creation of highly complex devices controlled by a single chip. 


\subsection{Lessons Learned}

During hardware testing of the motor controller, some problems arose when implementing the circuit at $115 \mathrm{~V}$. A low-power prototype had been constructed and tested on a single development board, and in this environment the AC and DC elements did not interfere. However, at high power some odd behavior was observed until the triac and inverting amplifier were separated from the breadboards where the PIC resided. Extensive hardware debugging and thorough measurements were needed to get the system working properly.

Another challenge to overcome was the slow execution of Simulink models. In order to be as authentic as possible, the simulations included some high-frequency timer and counter blocks. At one stage in development, a 250ns $(4 \mathrm{MHz})$ timer was incorporated in the digital PWM model, causing the full system simulation to run for over eight hours without finishing. Although some speed increases were made, specialized scripts had to be written so that multiple consecutive test cases could be run unattended. 


\section{References}

[1] "Harmonics Pollution"

http://www.metalect.co.nz/pfc/about_harmonics.htm (7 July 2004)

[2] "Motors: AC Induction Motors"

http://www.fpl.com/savings/energy_advisor/PA_35.html

[3] Nilsson, J. and S. Riedel. Electric Circuits, Fifth Ed. Addison-Wesley, 1996.

[4] "Induction Motor Control", L. M. Photonics Ltd.

http://www.lmphotonics.com/m_control.htm

[5] "Niagara Mohawk - Fundamentals"

http://www.niagaramohawk.com/busind/pwrqual/basics/basics.html (7 July 2004)

[6] DeDad, J. "When Does Poor PQ Cause Electronic Failures?" ECEM Online, 1 October 1999.

http://www.ecmweb.com/ar/electric_poor_pq_cause/

[7] "Harmonic Distortion"

http://cipco.apogee.net/pq/qcifhds.asp

[8] Energy Smart Company Website

http://www.energysmart.com

[9] "Technical Discussion", Power Saver Corporation

http://www.powersavercorp.com/faqs.html

[10] "Power Conditioner Also Provides Power Factor Correction" http://www.pnl.gov/TechFocus/pdfs/PowerCondTF.pdf (September 1998)

[11] Demidenko, S., J. Kells, K. Mercer, and D. Bailey. "Induction Motor Control Using Load Profiling Measurement and Processing." IEEE Instrumentation and Measurement Technology Conference Budapest, Hungary, May 21-23, 2001.

[12] dsPIC30F Digital Signal Controllers Product Family Brochure. http://ww1.microchip.com/downloads/en/DeviceDoc/dspbrochure_70095G.pdf

[13] Lathrop, O. "Digitally Control Power Factor Correction." Circuit Cellar Issue 174, January 2005 . 
[14] "High Performance Active Harmonic Filter."

http://www.activeharmonicfilters.com/High_Performance_Active_Harmon/

body_high_performance_active_harmon.html

[15] Hingorani, N. and L. Gyugyi. Understanding FACTS : Concepts and Technology of Flexible AC Transmission Systems. Wiley-IEEE Press, 1999.

[16] Habur, K. and D. O'Leary. "FACTS for Cost Effective and Reliable Transmission of Electrical Energy."

http://www.worldbank.org/html/fpd/em/transmission/facts_siemens.pdf

[17] Zemerick, S. Design of a Prototype Personal Static VAR Compensator. Master's Thesis, West Virginia University, 2002.

[18] Bach, D. United States Patent No. 5,592,062. United States Patent and Trademark Office, Washington, D.C. January 7, 1997.

[19] McConnell, R. Digital Electronics. Copyright 1993-1999.

[20] "Fundamental Characteristics of Thyristors." Teccor Electronics, Inc. http://www.web-ee.com/primers/files/an1001.pdf

[21] PIC18F452 Microcontroller Data Sheet, Microchip Technology, Inc. http://ww1.microchip.com/downloads/en/DeviceDoc/39564b.pdf

[22] MOC3051 Triac Driver Datasheet, Motorola, Inc. Accessed from DatasheetCatalog.com. http://www.ortodoxism.ro/datasheets/motorola/MOC3051.pdf

[23] BCR10CM Triac Datasheet, Mitsubishi Electric Corporation. Accessed from DatasheetCatalog.com.

http://www.datasheetcatalog.com/datasheets_pdf/B/C/R/1/BCR10CM.shtml

[24] Yokogawa PZ4000 Power Analyzer Product Website, Yokogawa Electric Corporation. http://www.yokogawa.com/tm/wtpz/pz4000/tm-pz4000_01.htm

[25] PIC C Compiler Manual, from CCSInfo.

Available at http://www.ccsinfo.com/ccscmanual.zip

[26] Power System Blockset for Simulink (now called SimPowerSystems) from The MathWorks. http://www.mathworks.com/access/helpdesk/help/toolbox/physmod/powersys

[27] "The TRIAC." All About Circuits, Vol. III, Ch. 7

http://www.allaboutcircuits.com/vol_3/chpt_7/6.html

[28] Ong, Chee-Mun. Dynamic Simulation of Electric Machinery Using MATLAB / Simulink. Prentice Hall, 1998.

[29] Kumar, S. "Simulation of a Single-Phase Active Power Line Conditioner Using Microsim Design Lab 8.0"

http://sureshks.netfirms.com/article/aplc/aplc.htm 


\section{Appendix A}

\section{Source Code}

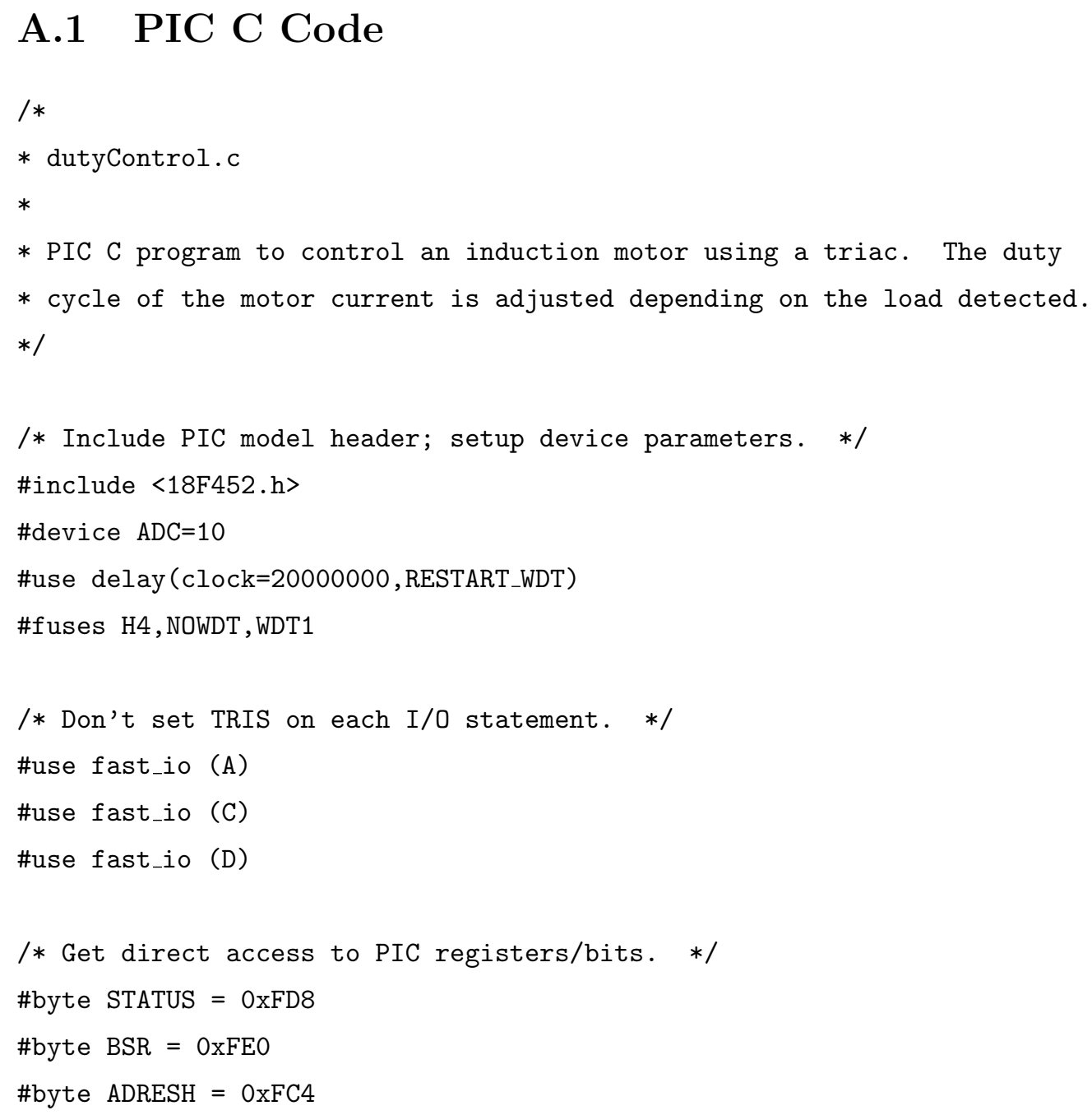




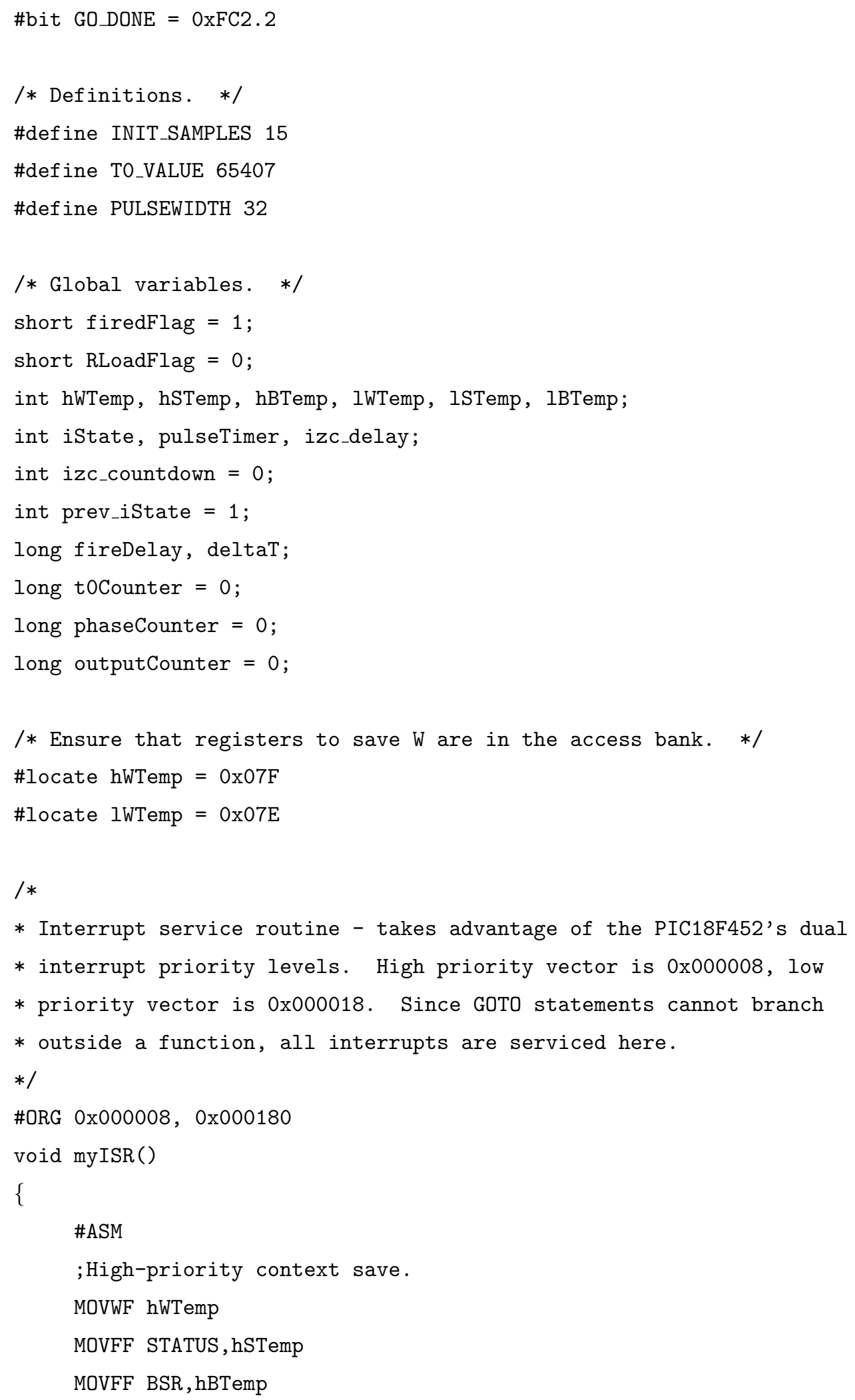


GOTO highIntSelect; Jump to interrupt polling routine

NOP

;Low-priority context save.

MOVWF IWTemp

MOVFF STATUS, ISTemp

MOVFF BSR, IBTemp

GOTO lowIntSelect ; Jump to interrupt polling routine

highContextRestore:

MOVFF hBTemp, BSR

MOVF hWTemp, W

MOVFF hSTemp, STATUS

RETFIE ; Return from high-priority interrupt

lowContextRestore:

MOVFF IBTemp, BSR

MOVF IWTemp,W

MOVFF ISTemp,STATUS

RETFIE ; Return from low-priority interrupt

highIntSelect:

BTFSC 0xFF2.2 ; Checking Timer0 interrupt flag

GOTO timerOISR

GOTO highContextRestore

lowIntSelect:

BTFSC 0xF9E. 6 ; Checking ADC interrupt flag

GOTO adcISR

GOTO lowContextRestore

\#ENDASM

/*

* Interrupt handler for the Analog-to-Digital Converter. Reads the

* value from the ADC, classifies it, and starts a new conversion.

$* /$

adcISR: 


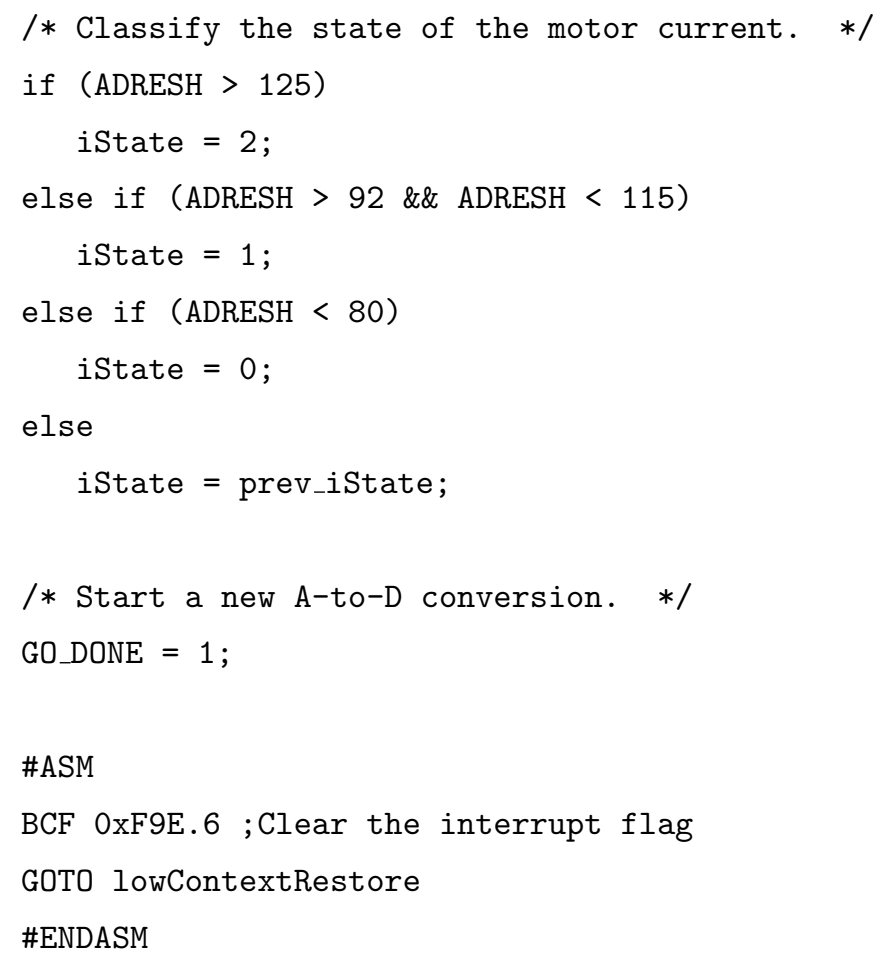




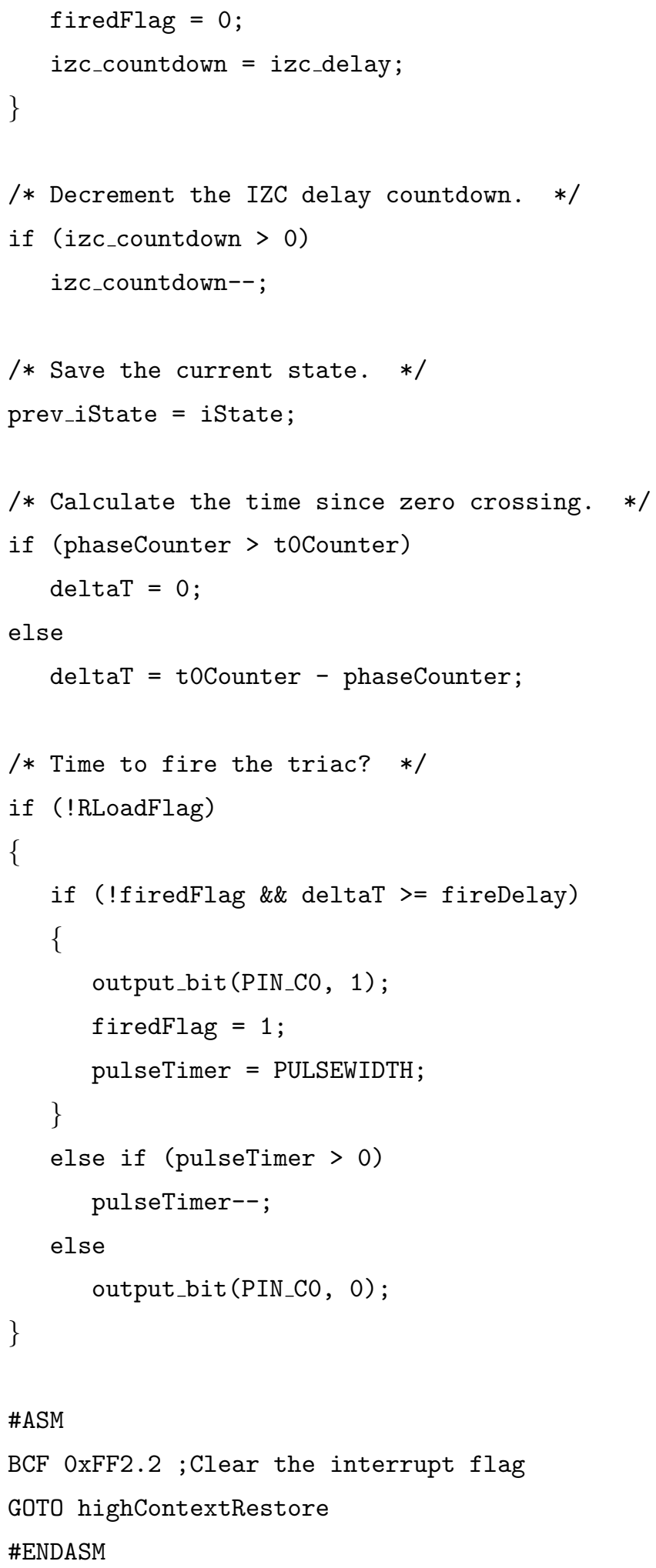




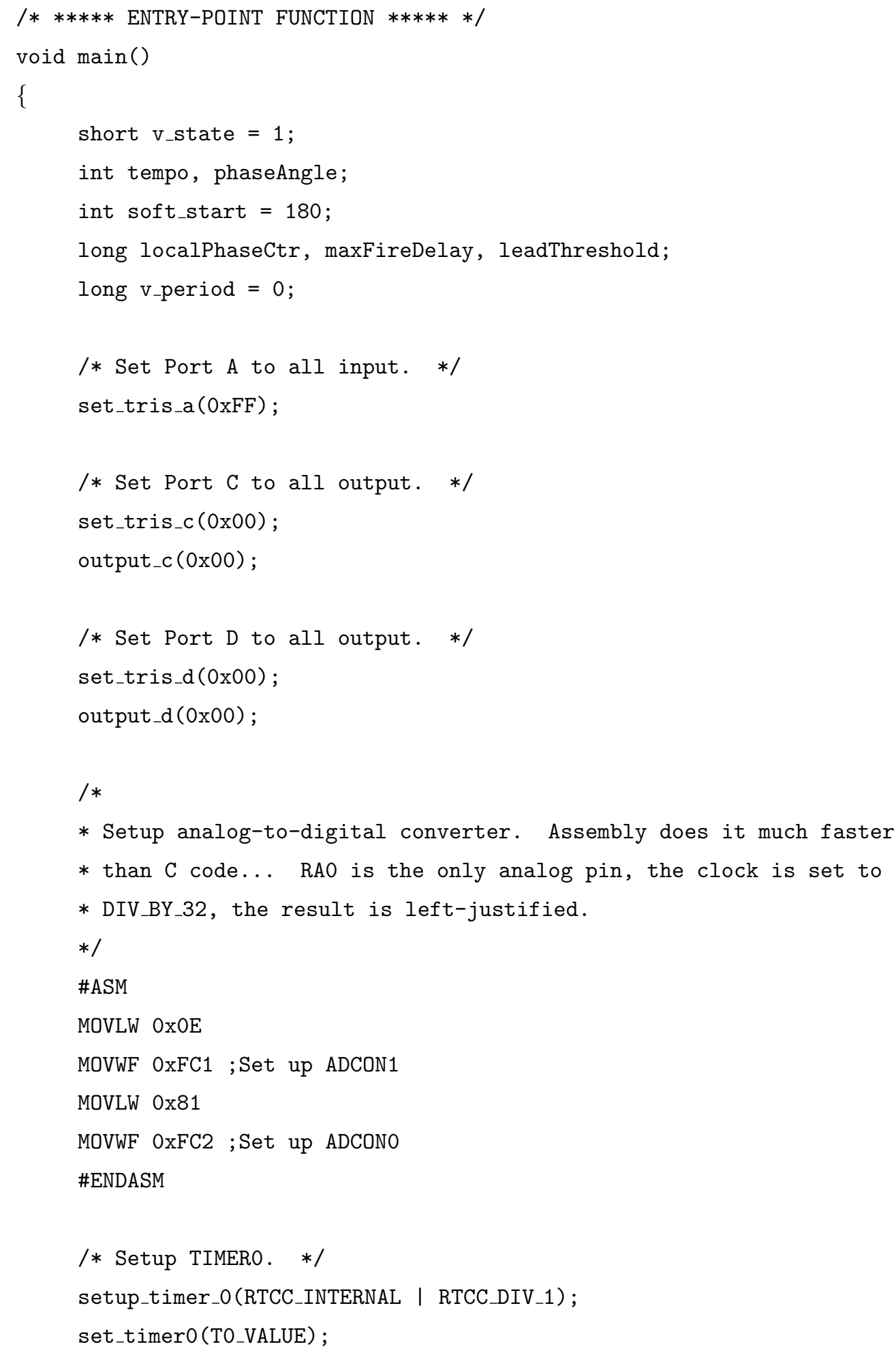




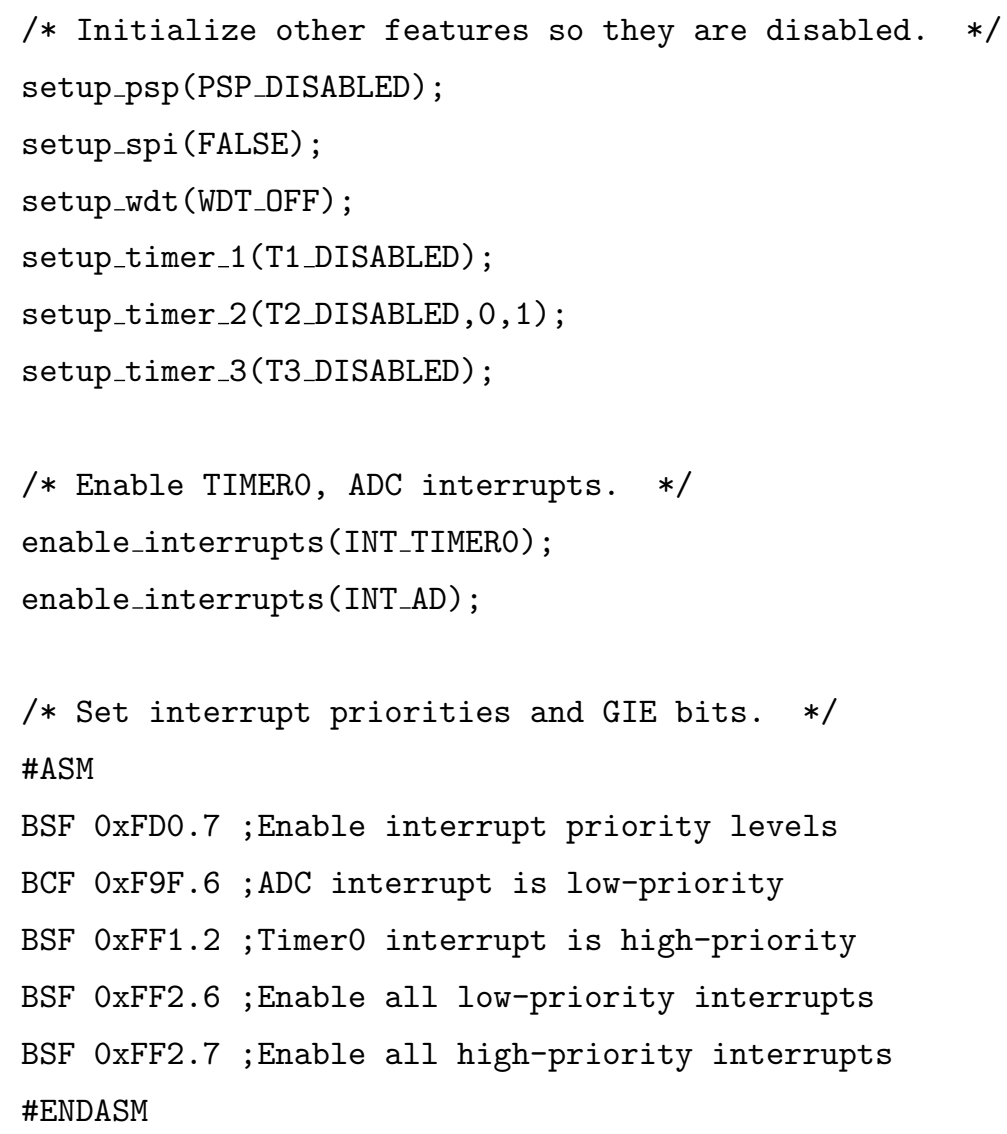




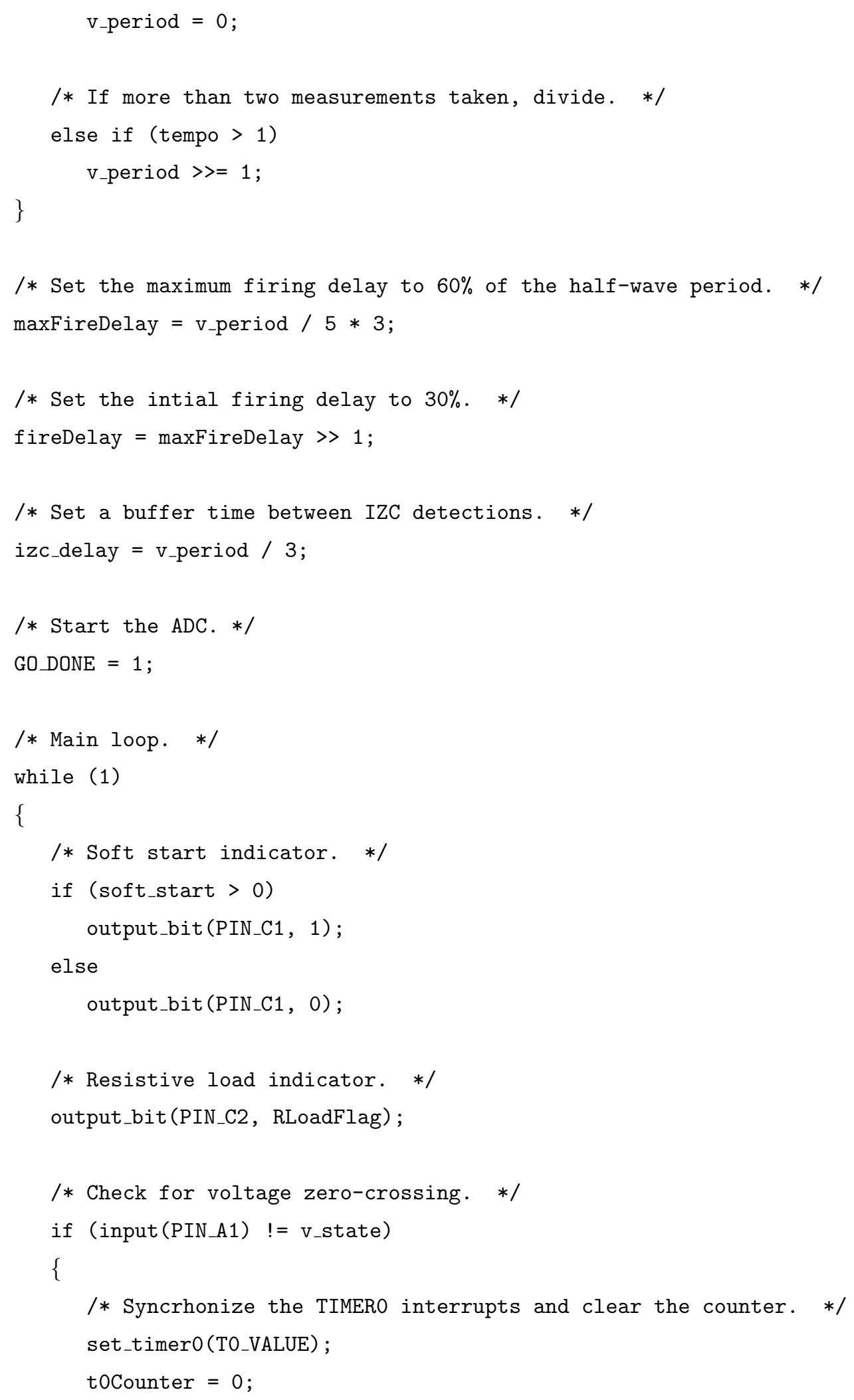




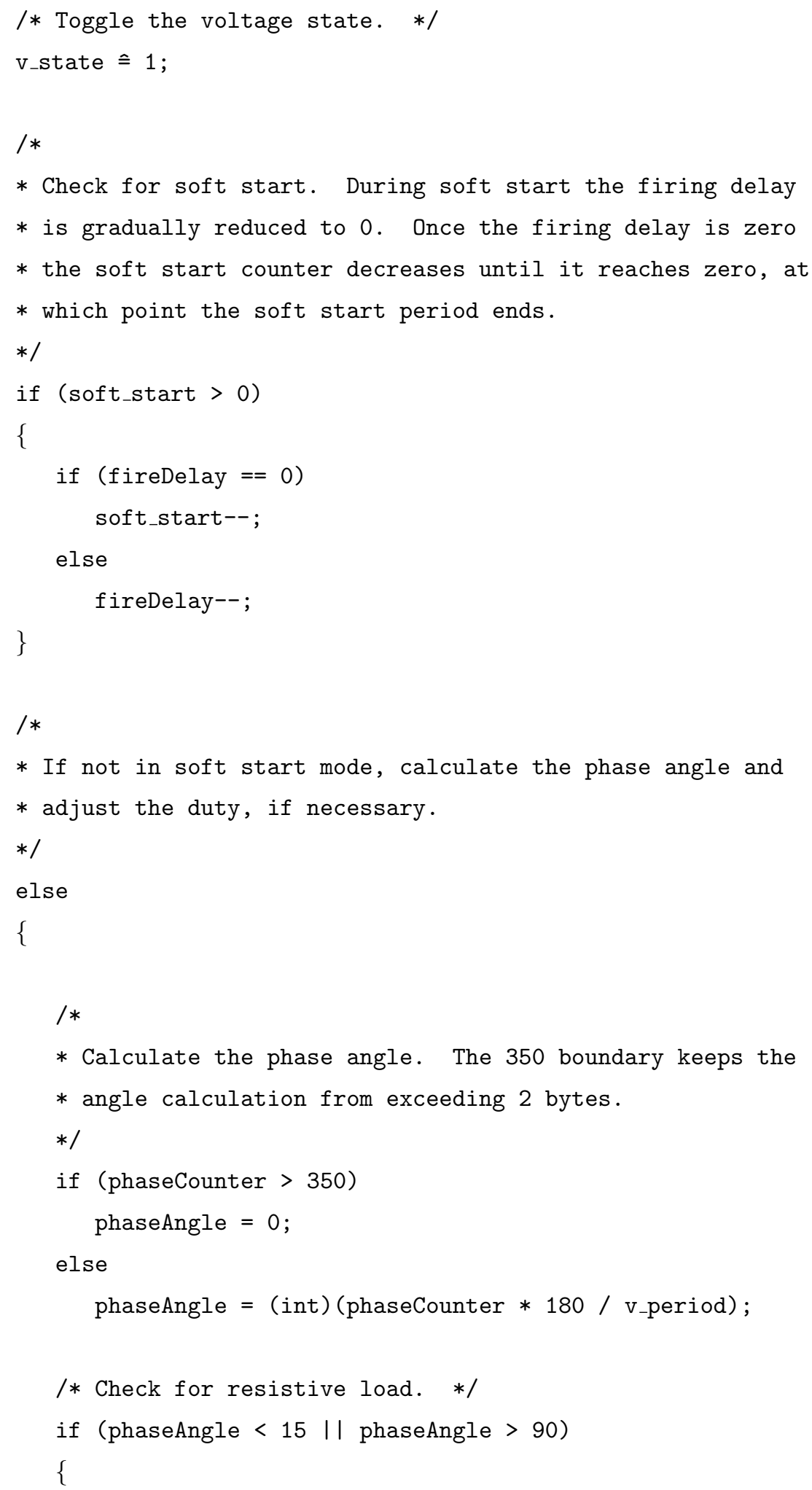




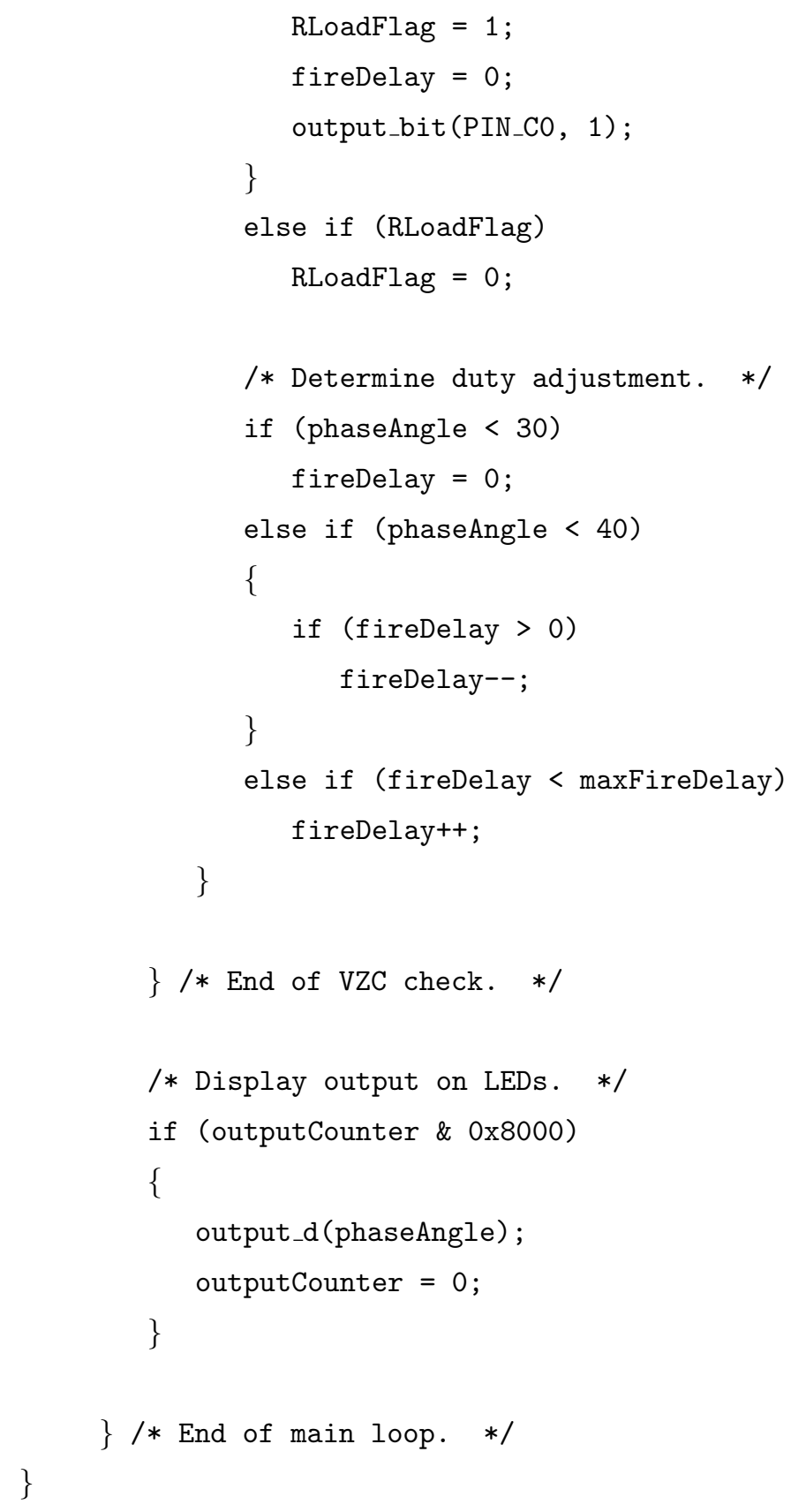

\section{A.2 MATLAB Code}

\section{A.2.1 calcFiringDelay.m}

function $\mathrm{y}=$ calcFiringDelay $(\mathrm{x})$

$\% \mathrm{y}=$ calcuFiringDelay $(\mathrm{x})$ 
$\%$

$\%$ Calculates the firing delay for the triac, where:

$\% \mathrm{x}=$ [phaseCounter, soft_start, RLoadFlag, firingDelay]

$\% \mathrm{y}=$ [firingDelay, soft_start, RLoadFlag]

$\%$

$\%$ This function is intended to be used with the Simulink model of the $\%$ motor control algorithm.

$\%$

$\%$ Define constants. A half-wave consists of 277 30-microsecond intervals.

$\%$ The maximum firing delay is $60 \%$, or 166 .

v_period $=277$;

maxFireDelay $=166$;

$\%$ Reassign input to named variables.

phaseCounter $=\mathrm{x}(1)$;

soft_start $=\mathrm{x}(2)$;

RLoadFlag $=\mathrm{x}(3)$;

firingDelay $=x(4)$;

$\%$ Check for soft start. During soft start the firing delay

$\%$ is gradually reduced to 0 . Once the firing delay is zero

$\%$ the soft start counter decreases until it reaches zero, at

$\%$ which point the soft start period ends.

if (soft_start >0)

if ( firingDelay $==0$ )

soft_start $=$ soft_start -1 ;

else

firingDelay $=$ firingDelay $-1 ;$

end

$\%$ If not in soft start mode, calculate the phase angle and

$\%$ adjust the duty, if necessary.

else

$\%$ Calculate the phase angle. The 350 boundary keeps the

$\%$ angle calculation from exceeding 2 bytes. 


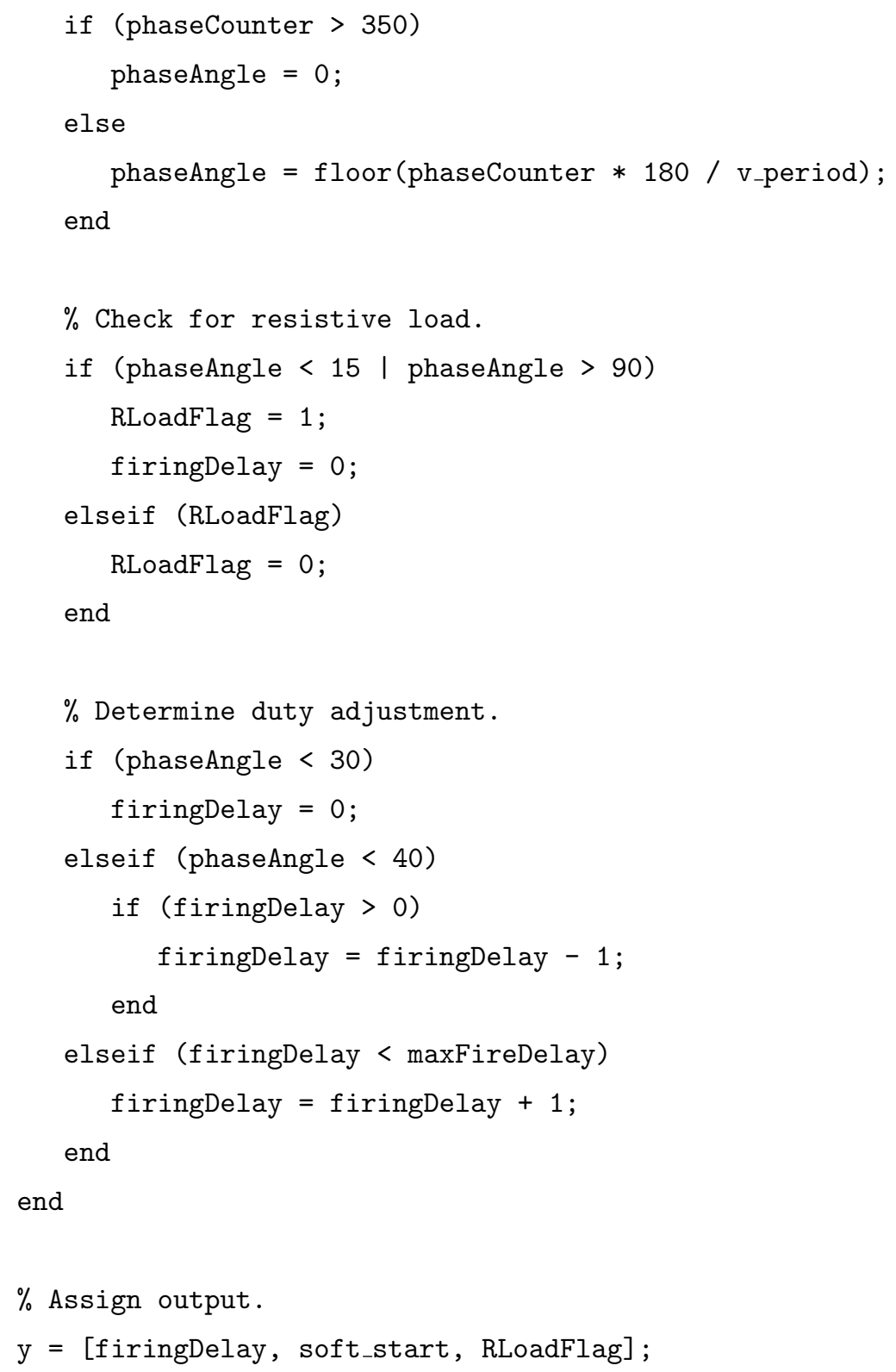

\section{A.2.2 gatePulseCtrl.m}

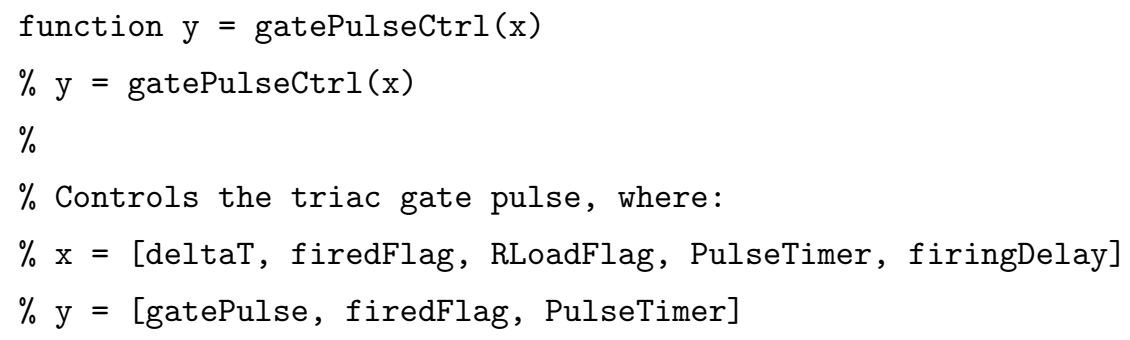


$\%$

$\%$ This function is intended to be used with the Simulink model of the $\%$ motor control algorithm.

$\%$

$\%$ Define constant for the pulse width.

PULSEWIDTH $=32$;

$\%$ Reassign input to named variables.

$\operatorname{deltaT}=x(1) ;$

firedFlag $=\mathrm{x}(2)$;

RLoadFlag $=\mathrm{x}(3)$;

PulseTimer $=\mathrm{x}(4)$;

firingDelay $=x(5)$;

$\%$ Determine the state of the gate pulse.

if ( RLoadFlag)

if ( firedFlag \& deltaT >= firingDelay)

gatePulse $=1$;

firedFlag $=1$;

PulseTimer $=$ PULSEWIDTH;

elseif (PulseTimer >0)

gatePulse $=1$;

PulseTimer $=$ PulseTimer -1 ;

else

gatePulse = 0 ;

end

else

gatePulse = 1 ;

end

$\%$ Assign output.

$\mathrm{y}=$ [gatePulse, firedFlag, PulseTimer $]$;

\section{A.2.3 genSineWaveTable.m}

function outVector $=$ genSinWaveTable $(f, t, s)$ 
$\%$ outVector $=$ genSinWaveTable $(f, t, s)$

$\%$

$\%$ Generates points along a sine wave with frequency $f$ (in $\mathrm{Hz}$ ) over

$\%$ time $t$ with s steps.

$\%$ Determine the time step size.

tStep $=t / s$;

$\%$ Calculate the angular frequency.

$\mathrm{w}=2 * \mathrm{f} * \mathrm{pi}$;

$\%$ Initialize the output vector.

outVector $=\operatorname{zeros}(1, \mathrm{~s})$;

$\%$ Main loop - for each time step calculate the sine value.

for $i=1: s$

$\operatorname{outVector}(i)=\sin \left((i-1) *\right.$ tStep $\left.^{*} \mathrm{w}\right)$;

end 